\title{
Complex Regulation of Protein Trafficking and Photoreceptor Cell Development by Small GTPases
}

\author{
Zachary C. Wright
}

Follow this and additional works at: https://researchrepository.wvu.edu/etd

\section{Recommended Citation}

Wright, Zachary C., "Complex Regulation of Protein Trafficking and Photoreceptor Cell Development by Small GTPases" (2016). Graduate Theses, Dissertations, and Problem Reports. 6979.

https://researchrepository.wvu.edu/etd/6979

This Dissertation is protected by copyright and/or related rights. It has been brought to you by the The Research Repository @ WVU with permission from the rights-holder(s). You are free to use this Dissertation in any way that is permitted by the copyright and related rights legislation that applies to your use. For other uses you must obtain permission from the rights-holder(s) directly, unless additional rights are indicated by a Creative Commons license in the record and/ or on the work itself. This Dissertation has been accepted for inclusion in WVU Graduate Theses, Dissertations, and Problem Reports collection by an authorized administrator of The Research Repository @ WVU.

For more information, please contact researchrepository@mail.wvu.edu. 


\title{
Complex Regulation of Protein Trafficking and Photoreceptor Cell Development by Small GTPases.
}

\author{
Zachary C. Wright \\ Dissertation submitted to the School of Medicine at \\ West Virginia University in partial fulfillment of \\ the requirements for the degree of \\ Doctor of Philosophy in \\ Pharmaceutical and Pharmacological Sciences \\ Visvanathan Ramamurthy, Ph.D., Chair \\ Ronald Gross, M.D. \\ Eric Tucker, Ph.D. \\ Jason Huber, Ph.D. \\ Maxim Sokolov, Ph.D.
}

Graduate Program in Pharmaceutical and Pharmacological Sciences

West Virginia University School of Medicine

Morgantown, West Virginia

2016

Keywords: ARL2, ARL3, Retinal Degeneration, Retinitis Pigmentosa, Protein Trafficking, Photoreceptor

Copyright 2016 Zachary Wright 


\title{
$\underline{\text { Abstract }}$ \\ Complex Regulation of Protein Trafficking and Photoreceptor Cell Development by Small GTPases.
}

\author{
Zachary C. Wright
}

Photoreceptor cells are specialized neurons optimized for the capture of light and the signal transduction to downstream cells that provides vision. Proper photoreceptor cell function depends heavily on efficient regulation of protein-protein interactions both during development and in the adult retina. Despite years of research on photoreceptor development and protein trafficking, the components vital to these processes are poorly understood. Interestingly, there are a number of small GTPases, which act as molecular switches, that are believed to play a role in regulating such interactions throughout the process of ciliogenesis, OS formation and protein trafficking. However, research into the role of these proteins in photoreceptor cells is in its infancy. Moreover, there are a growing number of discoveries linking small GTPases with photoreceptor mediated disease, such as ARL6, ARL2, ARL3, and RAB28. In this work I characterize the role of the small GTPases, ARL2 and ARL3, in the regulation of photoreceptor cell protein trafficking and OS development. In order to study these proteins in vivo, we utilized site-direct mutagenesis and transgenesis to create multiple animal models. Through these models, we have discovered that ARL2 and ARL3 serve different primary functions in vivo despite their high sequence similarity and sharing a number of binding partners. In Chapter 1 of this dissertation, we discuss the potential mechanisms and key players of photoreceptor cell ciliary development and protein trafficking. In Chapter 2, data is presented from the first animal model we generated expressing dominant active ARL3-Q71L. We further propose a mechanistic model for the role of ARL3 in prenylated protein trafficking in photoreceptor cells in which it acts to displace lipid modified cargo at the cilium. Chapter 3 focuses on data obtained from our dominant active ARL2-Q70L transgenic animal model. Here we discuss the potential role for ARL2 in ciliogenesis and OS formation in photoreceptor cells highlighting the novel observation that proper ARL2 function is necessary for cilia localization and axonemal extension. Finally, in Chapter 4 we discuss the data obtained during my dissertation and strategies to fill the gaps in knowledge that remain concerning the role of these small GTPases in photoreceptor cells. This work provides a strong foundation for the mechanisms of ARL2 and ARL3 action in photoreceptor cells. Complete elucidation of the components involved in photoreceptor protein trafficking and OS formation will provide the data necessary to generate novel therapeutics targeting the pathways underlying photoreceptor mediated blinding disease. 


\section{Acknowledgements}

This work would not be possible first and foremost without my advisor, Dr. Visvanathan Ramamurthy. I began fresh out of my second year of medical school where my schedule was regular and decided for me daily. Dr. Ramamurthy helped me understand how to manage my time properly and realize that very little happens as expected, at least in a $\mathrm{PhD}$, and how to plan accordingly. Our weekly meetings helped keep me on track toward larger goals by assessing the value of each experiment and properly planning it from the start. I must also acknowledge the help of Dr. Maxim Sokolov and Dr. Saravanan Kolandaivelu who provided much support and undivided attention whenever requested. Among my peers, I must first acknowledge my labmates Nachi Pendse, Abi Hayes, Tanya Dilan, and Jesse Sundar. It was lonely before they joined that lab and made grabbing a coffee much more enjoyable. I especially want to thank Dan Murphy, Joe Murphy, Brandon Smith and Michael Mueller for their friendship and support both academically and personally.

I would not be here without the love and support of my family. I must thank my parents for always supporting me without question and advocating for whatever made me happy. Likewise, my siblings, Zane, Derek, and Lauren, have been a continuous source of support and relief from the day-to-day struggles of graduate school. A special thanks and my love goes to my greatest advocate, Maggie Moreland, who has been there every single day of this rewarding and often stressful experience. She has supported without repose and deserves all my gratitude. 


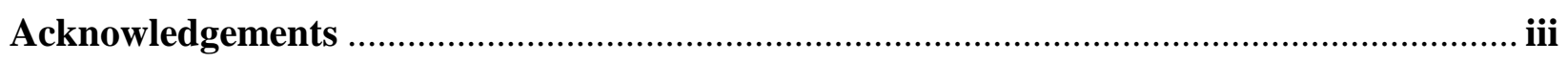

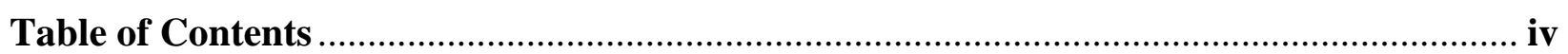

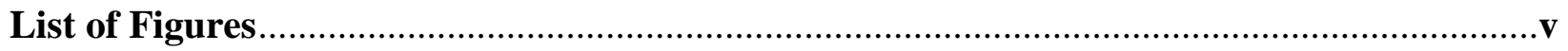

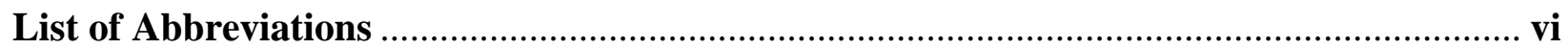

Chapter 1: Complex regulation of Photoreceptor Cell Biology by Small GTPases ................4

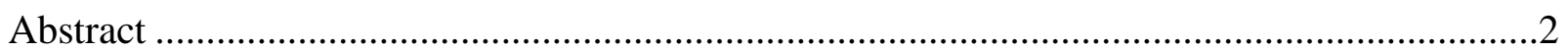

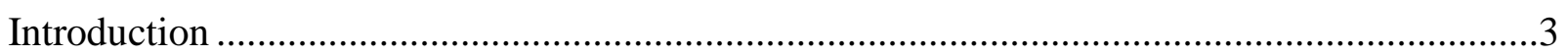

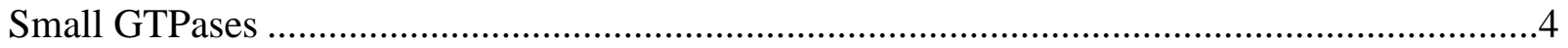

ARF4, RAB8, and RAB11 in Rhodopsin Trafficking ...........................................................6

ARL3 and ARL13b in the regulation of prenylated protein trafficking and OS formation..........8

ARL3 and ARL13b in ciliogenesis.................................................................................10

ARL3 in prenylated protein trafficking ......................................................................11

ARL3: Alternative functions.......................................................................................13

ARL2 and ARL2BP in photoreceptor OS development ...........................................................15

ARL6 in the regulation of photoreceptor cilium development and protein tafficking................17

RAB28 in blinding disease.............................................................................................19

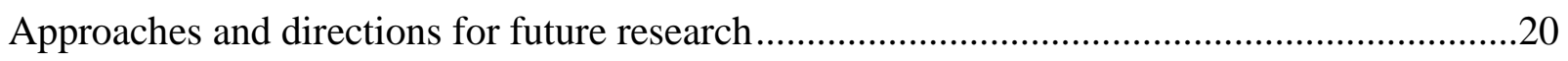

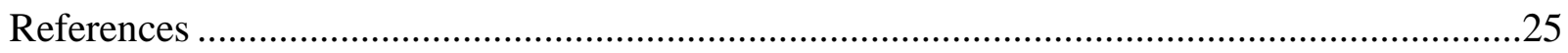

Chapter 2: ARL3 regulates trafficking of prenylated phototransduction proteins to the rod outer segment ............................................................................................................

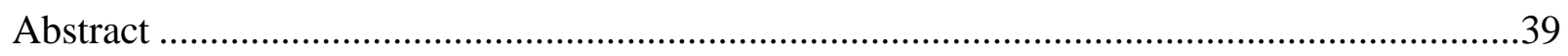

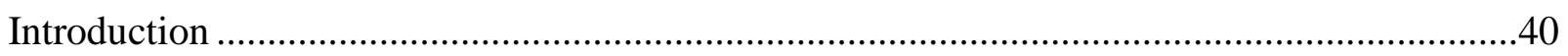

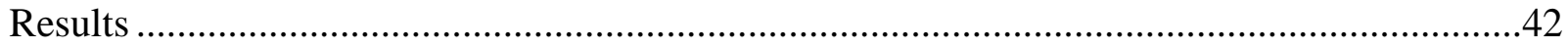

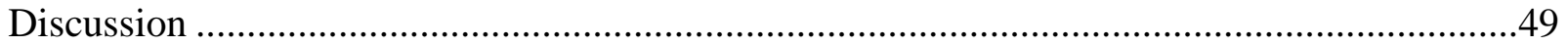

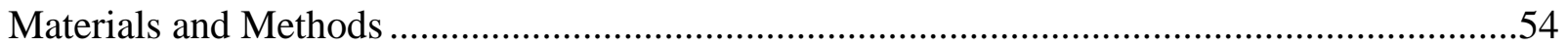

Acknowledgements .........................................................................................................60

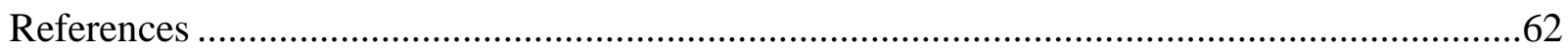

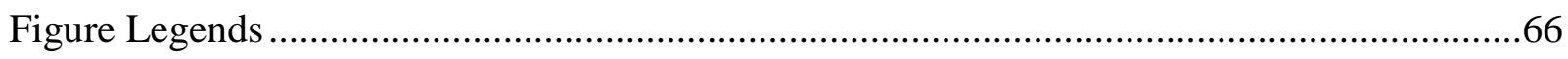

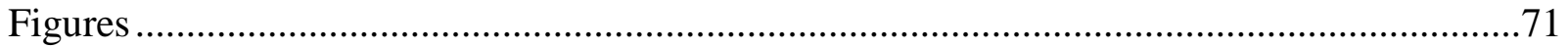

Chapter 3: ADP-Ribosylation Factor-Like 2 (ARL2) regulates photoreceptor cell ciliogenesis and rod $O S$ development. 


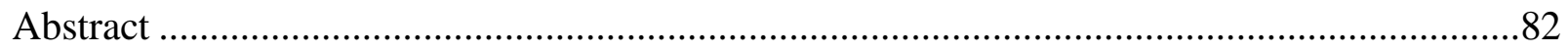

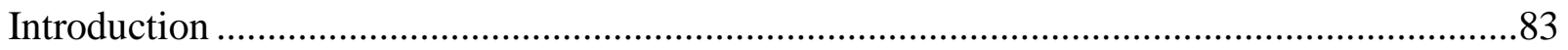

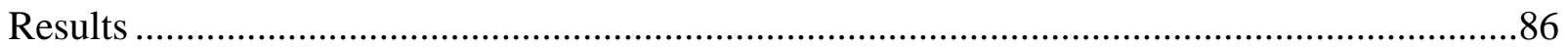

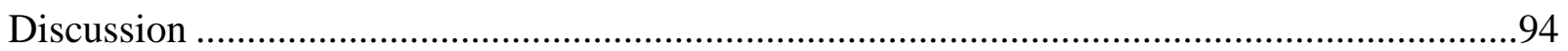

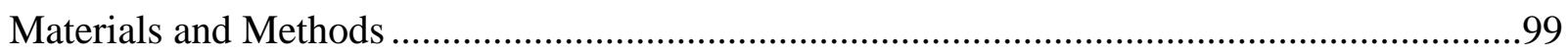

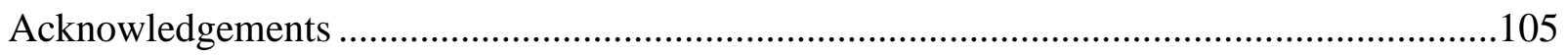

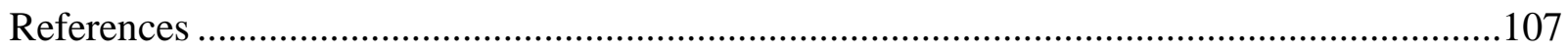

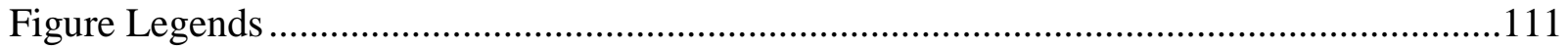

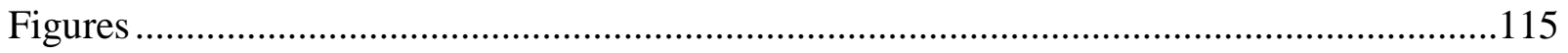

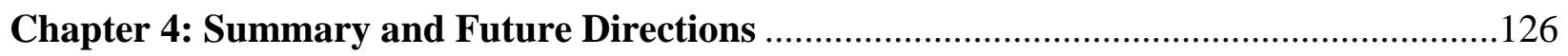

\section{$\underline{\text { List of Figures }}$}

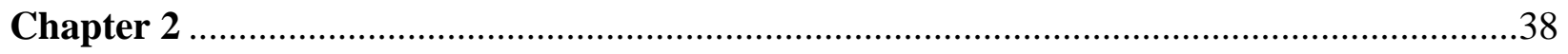

Figure 1. ARL3-Q71L Dominant Active Mutant Transgenic Model Generation.......................71

Figure 2. Progressive loss of rod photoreceptor function and degeneration in ARL3-Q71L

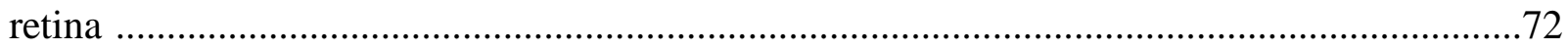

Figure 3. Normal elaboration of photoreceptor OS in ARL3-Q71L Mice..................................73

Figure 4. Expression of ARL3-Q71L reduces the levels of prenylated phototransduction

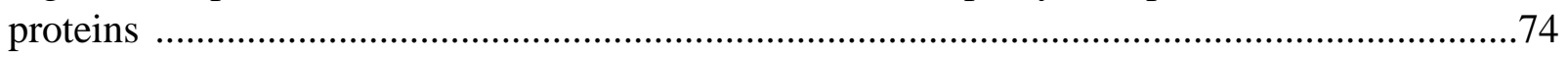

Figure 5. Loss of rod GRK1 and mislocalization of PDE6 in ARL3-Q71L mice .....................75

Figure 6. Progressive accumulation of rod PDE6 and colocalization of assembled rod PDE6

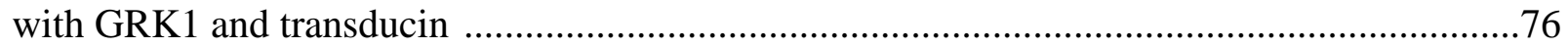

Figure 7. Accumulation of endosomal vesicles in the retina expressing ARL3-Q71L..............77

Figure 8. PrBP $\delta$ interacts with ARL3-Q71L in vivo..........................................................78

Figure 9. Defective migration of rod photoreceptor cells in ARL3-Q71L retina .......................79

Figure 10. Mechanistic Model for mistrafficking of prenylated proteins in ARL3-Q71L mouse

Chapter 3

Figure 1. Endogenous Arl2 Expression Profile and Generation of Dominant Active Mutant

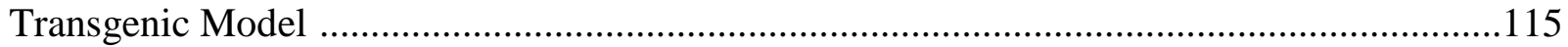

Figure 2. Decreased photoreceptor function in ARL2-Q70L mice..........................................116

Figure 3. Rod photoreceptor cells degenerate in animals expressing ARL2-Q70L.................117

Figure 4. Reduction of photoreceptor OS proteins in ARL2-Q70L animals ...........................118 
Figure 5. Abnormal elaboration of photoreceptor OS in ARL2-Q70L Mice.

Figure 6. Mislocalization of Rhodopsin in ARL2-Q70L animals.

Figure 7. ARL2-Q70L expression results in defective ciliogenesis and OS development in rod photoreceptor cells

Figure 8. Tubulin binding cofactor D (TBCD) interacts with ARL2 in vivo

Supplementary Figure 1. Intensity-response relationships for scotopic a-waves recorded at P16.

Supplementary Figure 2. Pan-reduction in photoreceptor OS proteins ARL2-Q70L animals 124 Supplementary Figure 3. Mislocalizatoin of Rhodopsin in ARL2-Q70L animals.

\section{List of Abbreviations}

RP - Retinitis Pigmentosa

ARL3 - ADP-Ribosylation Factor-Like 3

ARL2 - ADP-Ribosylation Factor-Like 2

ARL3-Q71L - transgenic dominant active ARL3

ARL2-Q70L - transgenic dominant active ARL2

TBCD - tubulin binding cofactor D

PDE6 - Phosphodiesterase 6

PDE6a - phosphodiesterase, alpha subunit

PDE6 $\beta$ - phosphodiesterase, beta subunit

PDE $\boldsymbol{\gamma}$ - phosphodiesterase, gamma subunit

GRK1 - Rhodopsin Kinase (G-protein receptor kinase 1)

XLRP - X-linked recessive retinitis pigmentosa

DAPI - 4',6-diamidino-2-phenylindole

ERG - electroretinogram

OS - outer segment

ROS - rod outer segment

COS - cone outer segment

IS - inner segment

ONL - outer nuclear layer

INL - inner nuclear layer 
IP - immunoprecipitation

CC - connecting cilium

RPE - retinal pigmented epithelium 


\section{Chapter 1: Literature Review}

Complex regulation of Photoreceptor Cell Biology by Small GTPases.

Zachary C. Wright ${ }^{1 \mathrm{a}}$ and Visvanathan Ramamurthy ${ }^{1 \mathrm{a}, \mathrm{b}, \mathrm{c*}}$

Departments of ${ }^{\mathrm{a}}$ Ophthalmology and ${ }^{\mathrm{b}}$ Biochemistry, ${ }^{\mathrm{c}}$ Center for Neuroscience, Robert C. Byrd Health Sciences Center, ${ }^{1}$ West Virginia University, Morgantown, West Virginia 26506, USA;

*Address Correspondence to: Visvanathan Ramamurthy, West Virginia University Eye Institute, One Medical Drive, E-363, Morgantown, WV 26506-9193, Tel: 304598 6940; Fax: 304-5986928; Email: ramamurthyv@wvumedicine.org 


\begin{abstract}
The photoreceptor cell sensory cilium is a complex organelle optimized for capturing light signal and transduction of that signal to downstream neurons. This specialized cilium requires a regulated series of interactions to produce the final functional outer segment with organized membrane discs loaded with phototransduction proteins. There is growing evidence that small GTPases play a major role in photoreceptor cell biology, specifically in the development and maintenance of the sensory cilium. Small GTPases are molecular switches that orchestrate molecular interactions by modifications of the guanine nucleotide binding status and these proteins are crucial to a wide range of cellular processes. Here we review the recent advances in our understanding of a subset of small GTPases underlying the processes of photoreceptor sensory cilium formation and protein targeting to the outer segment. A number of these small GTPases have been implicated in retinal degenerative diseases and syndromic ciliopathies. Understanding the basic mechanisms through which small GTPases act will provide insight into pathological states that will guide therapeutic intervention.
\end{abstract}




\section{INTRODUCTION}

Photoreceptor cells are highly specialized neurons that utilize a complex modified cilium, the photoreceptor outer segment (OS), to efficiently capture light (Pearring et al. 2013). This organelle poses major demands in regulation of developmental processes such as OS formation as well as maintenance of function by selective trafficking of OS proteins to their site of action. Cilia formation, i.e. ciliogenesis, in photoreceptor cells must be efficiently regulated so as to allow for creation of a functional, organized outer segment (OS) with stacked membrane discs that are packed with phototransduction and structural proteins necessary for sight. A number of diseases are the result of malformation of the OS and these diseases often occur because of an inherited defect in the genes involved in ciliary formation. These defects result in blinding disease as well as syndromic disorders known as ciliopathies, which result from a generalized defect in cilia of multiple organs. On the other hand, the demand for efficient protein trafficking in the mature OS is a result of the anatomy of the photoreceptor cell which has distinct compartments, i.e. the inner segment (IS) where OS proteins are synthesized and the OS where those proteins must function to capture light (Nachury, Seeley, and Jin 2010). Correspondingly, human disease may result from inefficiency in the process of protein trafficking in photoreceptor cells. Notably, mutations that disrupt the transport of phosphodiesterase 6 (PDE6), rhodopsin, or other phototransduction proteins are a major cause of the blinding disease, retinitis pigmentosa (RP) (Iannaccone et al. 2006; D. Y. Wang et al. 2005). Retinitis pigmentosa (RP) alone is responsible for vision loss in 1 in 4,000 people worldwide (Sung and Chuang, 2010). It is critical to realize that treatment for this disease is limited and clinical trials have produced very few promising results. Gene therapy approaches have been tested with some success, but have not yet produced overwhelming effectiveness (Petrs-Silva and Linden 2013; Rossmiller, Mao, and 
Lewin 2012). Despite the importance of OS development and efficient trafficking of proteins in photoreceptor cells, the mechanisms behind the regulation of these processes are poorly understood.

Cilia development and targeted protein trafficking require methods for regulating protein:protein and protein:lipid interactions a specific locations and timepoints. Small GTPases are a major family of proteins that are specialized to function based on the immediate subcellular environment making them optimized for this function. Small GTPases are known regulators of protein and vesicular trafficking as well as developmental processes in other cell types, however, they are understudied in the realm of photoreceptor cell biology. The aim of this review is to discuss the emerging role of small GTPases in the major processes of photoreceptor cell biology including OS/ciliary development and polarized trafficking of phototransduction machinery.

\section{SMALL GTPases}

Small GTPases are molecular switches that have a wide range of functions acting as key regulators in events such as proliferation, differentiation, cytoskeletal organization, and vesicular trafficking (Y. Li and Hu 2011). The superfamily of small GTPases is divided into five major subfamilies based on sequence similarity and functional homology (i.e. Ras, Rho, Rab, Arf/Arl, and Ran) (Weenerberg et al., 2005). These versatile proteins use a functional switching process that depends upon GTP hydrolysis to cycle between the GTP-bound "active" and GDP-bound “inactive” states, similar to heterotrimeric G proteins. The crucial aspect of their function in many processes is that the modulation of the "active" and "inactive" states is dependent upon the presence or absence of particular regulatory proteins that in some cases are required for the cycling (reviewed in Cherfils and Zeghouf 2013). Guanine nucleotide exchange factors (GEFs) facilitate the exchange of GDP for GTP and therefore activation, while GTPase activating 
proteins (GAPs) stimulate GTP hydrolytic activity of the small GTPase (reviewed in Cherfils and Zeghouf 2013). These modulators only activate or inactivate small GTPases in a specific context making them ideal for regulating complex protein-protein interactions as well as generating and maintaining membrane identity. These processes are especially important in photoreceptor neurons, which require complicated protein interactions in specific intracellular compartments, such as the connecting cilium or OS. Most proteins in the OS localize to a specific location such as the connecting cilium, the disc membrane or the plasma membrane, thereby requiring the trafficking to be highly selective. This exemplifies the typical scenario of small GTPase function, where it is active in one compartment, (e.g. the outer segment) and inactive in another (e.g. inner segment).

Modulation of a given small GTPase's guanine nucleotide binding state are just one component of its cellular functionality. Many of these proteins are highly homologous and share multiple domains that are conserved. These domains are related to the general small GTPase function as a molecular switch, while the remaining sequence determines the cellular processes that it may be involved in by dictating its effector proteins of localization. Some proteins can have very high protein sequence identity/similarity and share many of the same interacting partners, yet have different functions (e.g. ARL3 and ARL2). For example, ARL3 and ARL2 proteins have 53\% identity in primary sequence and a high degree of structural similarity yet serve different roles in multiple cells lines. Specifically, ARL3 loss results in failure of cytokinesis and an increase in acetylated- $\alpha$-tubulin, while in the same cell line, overexpression of dominant active ARL2 results in arrest in M phase along with microtubule obliteration (Zhou et al. 2006). While these proteins share some interacting proteins such as ARL2BP, TBCD, UNC119 (HRG4), and PrBP 8 , they also have specific interactors like retinitis pigmentosa protein 
2 (RP2) (for ARL3) and TBCC (for ARL2) (Burd, Strochlic, and Gangi Setty 2004; Veltel et al. 2008; Chen et al. 2016). The extent to which these similarities and differences play a role in vivo has yet to be determined. This example demonstrates the complexity of the interactions of small GTPases and that it requires years or decades of research to tease out the details and sequence of events of a given molecular pathway. Here we focus on the on the function of small GTPases in photoreceptor cell biology in order to highlight their functional diversity and demonstrate the methodology used to tease out molecular mechanisms underlying their function.

\section{ARF4, RAB8, and RAB11 IN RHODOPSIN TRAFFICKING}

Rhodopsin is capable of capturing photons and altering its conformation to begin an intracellular signaling cascade, known as phototransduction. It is a highly abundant 7-transmembrane protein with additional post-translational modifications including palmitoylation, phosphorylation, and glycosylation (Murray, Fliesler, and Al-Ubaidi 2009). Considering it is the first protein in the phototransduction cascade it has been studied extensively with respect to its physiological function, as well as the mechanisms involved in localization to its site of function in the OS. It is imperative that rhodopsin be selectively localized to the OS; this process requires a complex series of protein:protein and protein:lipid interactions regulated by a set of small GTPases. Rhodopsin trafficking involves at least three known small GTPases just in its transportation from the Golgi apparatus to the photoreceptor cilium. In this complex trafficking process rhodopsin is synthesized and processed in the endoplasmic reticulum (ER) and then sent to the Golgi apparatus for post-translational modification and sorting to the appropriate membrane compartment (J. Wang et al. 2012; Dusanka Deretic and Wang 2012; Dusanka Deretic 2013). This process of sorting is regulated by a number of proteins including Arf4, ASAP1, Rab11, Rabin8 and Rab8 (J. Wang et al. 2012). These proteins are primarily functional sets of small 
GTPases associated with their regulatory counterparts, GAPs and GEFs, which interact sequentially to bud rhodopsin-containing vesicles from trans-golgi network (TGN) and transport rhodopsin to the photoreceptor connecting cilium. The initial indication of ARF4 involvement was discovered through its interaction with the VXPX domain of rhodopsin which is necessary for trans-Golgi vesicle budding process (Dusanka Deretic et al. 2005). Subsequent studies demonstrated that upon ASAP1 (the COPI-like adaptor protein and Arf4 GAP) binding, Arf4 is inactivated and released, and Rab11 and FIP3 are recruited to regulate budding and sorting of rhodopsin-containing vesicles from the trans-Golgi network (TGN) (Mazelova et al. 2009). ASAP1 then aids in the recruitment of RAB8-GDP with ASAP1 and eventual formation of the Rab11-Rabin8-Rab8 complex with the addition of Rabin8 (the Rab8 GEF) near the basal body (Dusanka Deretic and Wang 2012; Hattula et al. 2002; Nachury et al. 2007). Rab8 is critical for rhodopsin vesicle passage into the connecting cilium region and fusion of those vesicles (D Deretic et al. 1995; Moritz et al. 2001). Further studies showed that Rabin8 GEF activity for Rab8 is stimulated by Rab11 binding (Knödler et al. 2010). This is an interesting case of divergence from less complex ciliary structures as photoreceptor cells contain an alternative GEF protein for Rab8 that is localized to the connecting cilium, that is, retinitis pigmentosa GTPase regulator (RPGR) (Murga-Zamalloa et al. 2010; Hong et al. 2001). The reason for this extra layer of regulation is unknown and deserves further study. It is possible that Rab8 is used as a conserved mechanism for transportation of proteins other than just rhodopsin and RPGR acts as the regulator in those scenarios.

This process was teased out over two decades of research, using multiple models and systems; thus providing an excellent example of building an elegant molecular model piece by piece. Furthermore, these studies spurred research of other disease-associated ciliary proteins 
with VXPX motifs in different cell types, such as polycystin-1 and polycystin-2 (Geng et al. 2006; Ward et al. 2011). This research supports the idea that the rhodopsin transport mechanism is conserved for a subset of ciliary proteins because the trafficking machinery in polycystin-1 trafficking is analogous, including Arf4, ASAP1, Rab11 and Rab6 (Ward et al. 2011). Additionally, the molecular pathogenesis underlying multiple blinding disease states can and have been ascertained by the mechanisms underlying this model. This complex set of interactions demonstrates the versatility of small GTPases involved in the trafficking of a single membrane embedded protein. Nonetheless the mechanism underlying the transportation of rhodopsin to the OS and final insertion into the disc membrane remains unclear.

It would be interesting to apply these same study techniques to examine the trafficking of other OS proteins. It is likely that the small GTPase interactions are conserved and are also responsible for trafficking of other transmembrane proteins (e.g. peripherin, ROM1, etc.). These proteins may in fact piggy-back on rhodopsin laden vesicles to their destination or the mechanism may involve a subset of common proteins along with unidentified substitutes for known proteins that serve some minor differences in regulation of transport.

\section{ARL3/ARL13B IN THE REGULATION OF PRENYLATED PROTEIN TRAFFICKING AND OS FORMATION}

The small GTPase, ADP-ribosylation factor-like 3 (ARL3), was identified as a ciliary protein by genomic analysis as well as being implicated in photoreceptor cilia through its discovered interaction with RP2, mutations of which result in X-linked retinitis pigmentosa (Avidor-Reiss et al. 2004; Bartolini et al. 2002). Very little was known at this time about ARL3 and the Arl family of small GTPases in general (Gillingham and Munro 2007). More recently, ARL13b has been identified as a potential GEF for ARL3 at the connecting cilium (Gotthardt et al. 2015). 
Furthermore, ARL13b mutations are associated with a common blinding disease associated syndrome called Joubert Syndrome (Miertzschke et al. 2014; Cantagrel et al. 2008). This association with cilia sparked research into its functional role in ciliary processes and more specifically in the development and associated processes of the modified cilium, the photoreceptor cell OS. Although initial research in photoreceptor cells demonstrated primarily ciliary localization, further study has indicated that ARL3 is present throughout the entire photoreceptor layer including the OS (Grayson et al. 2002; Z. C. Wright et al. 2016). Additionally, Arl3 deletion in mice results in cilia-related defects including cyst formation in the pancreas, liver, and kidney as well as abnormal photoreceptor development (Schrick et al. 2006). Follow-up studies demonstrated that ARL3 appears to play multiple roles throughout the life of the photoreceptor cell. The phenotypes associated with ARL3 dysfunction or loss in photoreceptor cells are dependent upon the timing of the deletion or loss of ARL3 function. For instance, floxed-Arl3 mice expressing retina-specific Cre embryonically demonstrate a similar phenotype to that seen in the complete KO mentioned above; that is, impaired development of the OS and fast degeneration of photoreceptor cells (Hanke-Gogokhia et al. 2016; Schrick et al. 2006). However, when Rhop-Cre (expressed at $\sim \mathrm{P} 4$ ) is expressed in these mice, photoreceptor cells develop normally and have progressive mislocalization of prenylated proteins and delayed cell death (Hanke-Gogokhia et al. 2016). Furthermore, expression of a dominant active mutant (ARL3-Q71L) under the same Rho promoter results in the progressive mislocalization of prenylated proteins to the IS and progressive degeneration with no defect in photoreceptor cell development (Z. C. Wright et al. 2016). Surprisingly, our studies also indicated that ARL3 plays a role in the process of nuclear migration of photoreceptor cells (Z. C. Wright et al. 2016). 
Moreover, this nuclear migration phenotype can be seen in the RP2 knockout mouse model (L. Li et al. 2013).

\section{ARL3 and ARL13b in Ciliogenesis}

Unfortunately, the reasons underlying the misdevelopment of the photoreceptor cell OS are widely unknown. In contrast to studies in vivo (see above), knockdown ARL3 in mammalian in vitro systems only affects transport to the cilium and not ciliary development (Lai et al. 2011). In addition, as with other small GTPases there are differences in phenotype associated with how the protein is manipulated. For instance, in C. elegans, knockout of the Arl3 gene results in defects in trafficking of sensory proteins to the cilium, but no ciliary deformations, while overexpression of the dominant active mutant leads to defects in ciliogenesis (Y. Li et al. 2010). Li et al. (2010) also implicated coordination between ARL3 and ARL13b (more detail later). They conclude that loss of ARL13b results in deformation of cilia in C. elegans and overexpression of dominant active ARL3 in these strains produces an additive effect. Additionally, deletion of Arl3 in Arl13b knockout strains partially rescues cilia effects. Further studies in C. elegans have demonstrated a potential causative relationship for misdevelopment of photoreceptor OS through the role of ARL3 in regulation of IFT proteins along with ARL13b (Y. Li et al. 2010). Although the molecular mechanisms are not completely understood, Arl3 may act though a HDAC6 pathway to regulate IFT complex integrity to have downstream effects on ciliogenesis (Y. Li et al. 2010). Mouse models have further implicated ARL3 and ARL13b in the process of photoreceptor OS morphogenesis. As mentioned above, embryonic knockout of Arl3 in photoreceptor cells results in aberrant development of OS and early cell death (Hanke-Gogokhia et al. 2016; Schrick et al. 2006). Similarly, Arl13b-/- mouse embryos exhibit shortened nodal cilia and abnormal axoneme structure (Caspary, Larkins, and Anderson 2007). More recent data suggests that ARL13b acts as 
the GEF of ARL3 (Gotthardt et al. 2015). This is quite pertinent information for the following section on the role of these proteins in trafficking to the OS, however, it is difficult to interpret in the process of OS development.

From the data provided in the in vitro systems and non-mammalian systems it is quite difficult to build a clear picture of how these proteins contribute to the process of ciliogenesis in the setting of photoreceptor cells. A complete understanding of their function in photoreceptor cell OS formation will require a more detailed look in in vivo models containing photoreceptor cells. For example, assessment of conditional and inducible knockout of Arl13b as well as transgenic expression of dominant active ARL13b in mice would provide the foundation for further research. These animal models along with a deeper look into the interacting partners of ARL3 and ARL13b in photoreceptor cells. Finally, the creation of a heterologous system for photoreceptor research would provide breakthroughs in the understanding of the field.

\section{ARL3 in Prenylated Protein Trafficking}

As stated above, the interacting proteins of each small GTPase determine its cellular role. More specifically, the localization of the GEF and GAP determine where and when it interacts with its effector proteins. This combined with in vitro data showing the resulting functional outcome of these interactions helped produce a new model of protein trafficking in photoreceptor cells. In this case ARL3 interacts with RP2 (the ARL3 GAP), ARL13b (the ARL3 GEF), and PrBP6 $\delta$ (the primary ARL3 effector) in the trafficking of PDE6 and other prenylated proteins in the phototransduction cascade. RP2 was identified as an interacting protein that binds preferentially to the ARL3-GTP bound state (Bartolini et al. 2002; Kühnel et al. 2006). 
$\mathrm{RP} 2, \operatorname{PrBP} \delta$, and ARL13b proteins were implicated in the transport of PDE6 as well as other lipid-modified proteins through PDE68 (Ismail et al. 2011; Gotthardt et al. 2015). Arl3 was found to be an effector protein of RP2 which acts as a GAP thereby inactivating Arl3 (Kühnel et al. 2006; Schwarz et al. 2012). The RP2 gene is defective in X-linked RP (Branham et al. 2012), and it implied that Arl3 would be left activated. This can be inferred from experiments that show a defect in RP2 function results in the same phenotype as a constitutively active Arl3 mutant (i.e. always GTP-bound) (Evans et al. 2010). Arl3 is primarily localized to throughout the rod and cone photoreceptor layer including the OS with some potentially higher concentrations at the connecting cilium (Grayson et al. 2002; Z. C. Wright et al. 2016). From these studies it appears that Arl3 plays a role in Golgi cohesion and vesicular transport of proteins to the photoreceptor cell connecting cilium (Schwarz et al., 2012). Proteomics using cultured cells and in vitro protein binding assays have revealed that Arl3 regulates the binding of some prenylated and myristoylated proteins to their corresponding cargo chaperone, i.e. PDE6 $\delta$ or Unc119. More specifically, Arl3 is thought to facilitate the release of Unc119-bound myristoylated proteins (e.g. transducin- $\alpha$ (G $\alpha$ subunit)) and PDE6 $\delta$-bound farnesylated proteins (e.g. G-protein coupled receptor kinase 1 (GRK1)) (Ismail et al., 2012; Wright et al., 2011). However, similar interacting intermediates have yet to be identified in the trafficking of PDE6 to the OS in photoreceptor cells.

Based on the results obtained in recent studies (Hanke-Gogokhia et al. 2016; Houbin Zhang et al. 2012; Z. C. Wright et al. 2016; Ismail et al. 2012; Ismail et al. 2011), we have developed a mechanistic model of ARL3 function in photoreceptor cell prenylated protein trafficking. PDE6, GRK1, and transducin begin their post-translation process with the addition of a prenyl group by a specific prenyl transferase. After prenyl addition, these proteins are targeted 
to ER to complete the last two steps of prenyl processing, endoproteolysis and methylation, which are mediated by ER-membrane bound enzymes. It is likely that $\operatorname{PrBP} \delta$ and its functional homologs can retrieve the prenylated proteins from endomembranes for further transport to the destination membrane (e.g. periciliary membrane or OS disc) similar to the proposed transport of K-ras from the ER to the plasma membrane (Chandra et al. 2012; Apolloni et al. 2000). After activation at the connecting cilium by its proposed GEF ARL13b, ARL3 stimulates release of prenylated cargo from $\operatorname{PrBP} \delta$. Because ARL13b is thought to be localized at the connecting cilium and OS, it suggests that ARL3-mediated release of cargo occurs at those locations. The remaining ARL3-PrBP $\delta$ complex can then be dissociated after inactivation of ARL3 by RP2, which facilitates hydrolysis of GTP to GDP.

This model was the result of over a decade of research from multiple in vitro to in vivo models. Importantly, it only illustrates a portion of the function of ARL13b and ARL3 as these proteins appear to have multiple functional roles in photoreceptor cells. In addition, there is a discrepancy in the length of time that each of these models produces phenotypes. For example, mice lacking $\operatorname{PrBP} \delta$ retina have much slower degeneration maintaining $\sim 5$ photoreceptor nuclear layers at six months of age (H Zhang et al. 2007). Compare this to knockout or overexpression of dominant active ARL3 at P5 which results in almost complete degeneration by two months (Z. C. Wright et al. 2016; Hanke-Gogokhia et al. 2016). These discrepancies imply that ARL3 has functionality beyond interacting with $\operatorname{PrBP} \delta$. Further studies to solidify this molecular model could utilize inducible knockout techniques that delete $\operatorname{PrBP} \delta$, ARL3, RP2, or ARL13b in adult mouse retina. This would isolate the trafficking phenotype from any artifacts conferred by developmental defects of the OS.

\section{ARL3: Alternative Functions}


One interesting aspect of small GTPases is the diverse range of functional pathways that they can be involved in. ARL3 has a number of proposed pathways and these do not necessarily involve interaction with the same proteins. As mentioned above, ARL3-Q71L expression results in the altered migration of rod photoreceptor cells (Z. C. Wright et al. 2016). This is interesting because there is only a subset of cells that do not migrate into the ONL, but instead remain in the INL. This is likely the result of onset of relatively late onset of transgenic ARL3-Q71L expression $(\sim \mathrm{P} 4)$ after major lamination of the retina is already underway. Additionally, this phenotype was observed in RP2 null mice, which demonstrate a subset of mislocalized photoreceptor nuclei in the INL although this was not specifically mentioned in the text (L. Li et al. 2013). It would be interesting to follow up on this phenotype to determine what specific proteins ARL3 preferentially interacts with at this stage of development. Notably, depletion of what is thought of as the primary effector protein, $\operatorname{PrBP} \delta$, in mouse photoreceptor cells does not recapitulate this phenotype, suggesting an alternative mechanism of action. This mechanism could potentially involve prenylated cargo release (e.g. such as nuclear lamins), but through an unidentified $\operatorname{PrBP} \delta$ homolog. In addition to this molecular function, ARL3 has been identified as a player in the regulation of release of dynactin complexes from dynein along with dynein light chain LC8 (M. Jin et al. 2014). This study also showed that knockdown of ARL3 by siRNA resulted in abnormal mislocalization of dynein, dynactin and organelles that are localized by those proteins. Finally, ARL3 has been implicated in maintaining Golgi cohesion and trafficking of IFTcontaining vesicles to the base of the cilium in vitro as well as taking part in a sorting module composed of Rabep1, GGA1 and ARL3 that binds to polycytin 1 at the Golgi for trafficking to cilia (Kim et al. 2014; Evans et al. 2010). 
These data support the conclusion that ARL3 has many potential functions as assessed in multiple species and cell lines. However, it appears that the primary functions in photoreceptor cells include OS development and trafficking of prenylated proteins. Bearing in mind that complete knockout of Arl3 in mice is lethal (Schrick et al. 2006), it is important to consider these other functions as potential mechanisms of the pathogenesis underlying the loss of ARL3 in other effected organs, such as the liver, kidney, and pancreas.

\section{ARL2 AND ARLBP IN PHOTORECEPTOR OS DEVELOPMENT}

The ARL3 homolog, ARL2, has recently been implicated in the processes of ciliogenesis and OS formation in photoreceptor cells (Davidson et al. 2013). It was found that mutations in ARL2 binding protein (ARL2BP, a.k.a. Binder of ARL Two (BART)) cause an autosomal-recessive form of retinitis pigmentosa. ARL2BP is localized to the basal body and cilium-associated centriole of photoreceptor cells as well as the periciliary ridge region (Davidson et al. 2013). As suggested by its name, ARL2BP is a major binding protein of ARL2, although the functional aspects of this interaction remain unclear. Davidson et al. (2013) has implicated both ARL2BP and ARL2 in the process of ciliogenesis by showing that depletion of either by siRNA knockdown in ARPE19 cells results in shortened cilia. Additionally, the ARL2BP mutation associated with RP, p.Met45Arg amino acid substitution, results in a significant decrease in the binding affinity to ARL2, suggesting that loss of this interaction is potentially responsible for the RP phenotype in these patients (Davidson et al. 2013). Furthermore, ARL2BP localization to the basal body was lost in ARPE19 cell lines after depletion of ARL2 and in patient epithelial cell lines with the p.Met45Arg mutation (Davidson et al. 2013). These data suggest that the interaction of ARL2 and ARL2BP is necessary for proper localization to the basal body. However, it is unclear whether ARL2BP or ARL2 has a major function associated with basal 
body or centriolar dynamics and whether one or the other is simply necessary for proper localization. It is important to note that ARL2 has previously been implicated in processes associated with microtubule and tubulin dynamics and these functions appear to be mediated at centrosomes where it is localized (Zhou et al. 2006; Mori and Toda 2013; Tian, Thomas, and Cowan 2010). More recent studies have teased out a mechanism through which ARL2 may affect microtubule dynamics in vivo. Specifically, ARL2-GTP interacts with TBCD and TBCE to form the "TBC-DEG" complex which is able to then catalytically manipulate $\alpha \beta$-Tubulin dimers driving potentially both biogenesis and degradation of those dimers by modulation of the ARL2 GTPase activity. It is thought that the high energy needed to dissociate $\alpha \beta$-tubulin dimers or release soluble polymerizable $\alpha \beta$-tubulin dimers is supplied by the GTP hydrolysis of ARL2 that is facilitated by its GAP tubulin binding cofactor C (TBCC). The conclusion is that ARL2 in association with TBC-DEG regulates the soluble pool of $\alpha$ - and $\beta$-tubulin and therefore the dynamics of microtubules that use that tubulin (Nithiananatham et al. 2015). However, it is important to note that ARL2BP does not currently have a proposed role in these models. Moreover, in vitro studies have shown that although ARL2BP is colocalized with ARL2 in the centrosome, specifically in the pericentriolar matrix (PCM), overexpression or loss of ARL2BP does not cause the defects in tubulin polymerization or centrosomal fragmentation that occur upon expression of dominant active ARL2-Q70L (Zhou et al. 2006). It should be noted that these results are specific to centrosomes and not cilia-associated centrioles. This suggests that ARL2BP and ARL2 may have exclusive co-dependent functions in cilia-associated centrioles only during specific stages of cell development. In order to understand this interaction in the context of the photoreceptor cell basal body, ciliary associated centriole, connecting cilium, and 
OS, we must characterize their interaction in ciliated cell lines completely as well as determine the phenotypes associated with loss in photoreceptor-specific knockouts or transgenic lines.

ARL2 versus ARL3 function demonstrates a unique case of divergence of function of similar proteins. With 53\% sequence identity and 63\% similarity as well as a number of common binding proteins, it is hard to imagine that they have vastly different functions. However, minor differences in the way these proteins interact with their shared binding factors as well as interactions with uncommon binding partners likely decide the functional fate. For example, ARL3 and ARL2 both interact directly with carrier protein UNC119, which is thought to regulate trafficking of myristoylated cargo, however, the outcome of this interaction is different. Specifically, binding of ARL3-GTP to an UNC119-Cargo complex results in a conformational change in UNC119 that causes release of the cargo. However, using this same experimental paradigm ARL2-GTP cannot stimulate release of cargo (K. J. Wright et al. 2011). This demonstrates that although similar small GTPases may have common interacting partners and a high degree of sequence homology they do not necessarily confer the same functional relationship.

\section{ARL6 IN THE REGULATION OF PHOTORECPTOR CILIUM DEVELOPMENT AND PROTEIN TRAFFICKING}

ADP-ribosylation factor-like 6 (ARL6) (a.k.a. BBS3) was the first identified small GTPase associated with human ciliopathy. Specifically it is associated with the syndromic disorder Bardet-Biedel Syndrome, which is characterized by obesity, cognitive impairment, renal dysfunction, polydactyly and retinitis pigmentosa, all related to dysfunction of primary cilia in those organs (Chiang et al. 2004; Fan et al. 2004). The Arl6-/- mouse model corresponds to the human BBS disease state with ciliary defects and associated phenotypes (Q. Zhang, Nishimura, 
Seo, Vogel, Morgan, Searby, et al. 2011). Most of the disease causing mutations are clustered around the GTP binding region of ARL6 resulting in its inability to effectively bind nucleotides, thereby disrupting its cycling state and the ability to bind to and act on effector proteins (Wiens et al. 2010). These data led to further studies into the role of ARL6 in cilia function. A number of previous results are species specific making it difficult to determine the overall function of ARL6 in photoreceptor cells. For example, studies in C. elegans suggest that it is involved in ciliary sensory function, but not ciliogenesis (Blacque et al. 2004). However, studies in mammalian cell systems shows that overexpression of ARL6 can result in disassembly of the cilium (Wiens et al. 2010). Additionally, there is yet another instance of divergence of phenotype based on the method of disrupting the small GTPase where expression of the dominant active ARL6-Q73L results in an increase in cilia formation along with an increase in cilia length (Wiens et al. 2010).In explanation to these differences in observed ARL6 functions, studies of the Arl6 deletion mouse model demonstrate that ARL6 has phenotypes in common with and unique from other BBSome protein mutants (Q. Zhang, Nishimura, Seo, Vogel, Morgan, and Searby 2011).

ARL6 interaction with the BBSome has been somewhat difficult to understand simply because the BBSome is such a complex protein structure that is not fully understood. The BBSome is composed 7 highly conserved BBS subunits BBS1, BBS2, BBS4, BBS5, BBS7, BBS8, and BBS9 along with BBIP10 (Nachury et al. 2007; Loktev et al. 2008). ARL6-deficient cells maintain an intact BBSome core complex, and therefore ARL6 does not contribute to its assembly (Q. Zhang, Nishimura, Seo, Vogel, Morgan, Searby, et al. 2011). However, ARL6 is required for the localization of the BBSome to the cilium as well as other ciliary proteins to the ciliary membrane (e.g. melanin concentrating hormone receptor 1) (Q. Zhang, Nishimura, Seo, Vogel, Morgan, Searby, et al. 2011). Additional mechanistic data suggest that ARL6 activation 
results in the assembly of a BBSome/ARL6 coat on membrane surfaces, which appears to sort proteins for entrance into the cilium (H. Jin et al. 2010). On the other hand, ARL6 appears to be a regulator of export of ciliary proteins such as the Hedgehog signaling receptors Patched 1 and Smoothened out of the cilium (Q. Zhang, Nishimura, Seo, Vogel, Morgan, Searby, et al. 2011; Q. Zhang et al. 2012). Interestingly, new animal studies demonstrate that the BBSome may function to either exclude non-OS proteins by an export mechanism as above or possibly by preventing entry into the OS (Datta et al. 2015). This provides an indication that the BBSome coordinated with proper targeting/function with ARL6 may provide some sort of regulation for movement of proteins to the base of the connecting cilium (from the OS) or out of the photoreceptor OS in addition to its function of targeting proteins from the IS to the cilium. ARL6 provides another example of the complexity of small GTPase function and how those functions may differ depending on the system or the intracellular context.

\section{RAB28 IN BLINDING DISEASE}

RAB28 is a member of the Rab (Ras-related in brain) subfamily of small GTPases. It was only recently implicated in photoreceptor function through a genomic analysis in multiple families identifying mutations in the RAB28 gene that result in cone-rod dystrophy (Roosing et al. 2013; Roosing et al. 2014; Riveiro-Álvarez et al. 2015). Rab GTPases are involved in a wide range of cellular processes mostly involving dynamics of vesicle trafficking, vesicle fusion, and membrane fission events. However, whether Rab28 plays a role in these processes is yet to be determined. Interestingly, Rab28 is the first known blinding-disease associated Rab GTPase that is prenylated. Note that Rab28 is localized to the photoreceptor cilium and thus may be involved in ciliary transport mechanisms. Other Rab proteins that have similar localization are involved in ciliary transport such as Rab8 and Rab11 as well as Rab23. Specifially, Rab23 regulates the 
turnover of Smo (smoothened) in the cilium (Boehlke et al. 2010). Based on localization data and the general role of Rab small GTPases it is possible that Rab28 plays a role in protein transport at the connecting cilium whether in the form of vesicle fusion, movement of proteins through the cilium, or targeting of proteins to the connecting cilium.

Elucidating its interacting partners is necessary in identifying the role of Rab28 at the photoreceptor connecting cilium. Additionally, dominant active and knockout animal models will provide major insight into the functions of this protein. Surprisingly, mutation of this protein only causes retinitis pigmentosa with no other observed phenotypes in humans. This suggests that it may have photoreceptor specific functions that are separate from other cell types or that there are proteins with redundant functions in those cells and not in photoreceptor cells. There are small number of examples of mutations in ubiquitously expressed proteins that lead to a photoreceptor specific phenotype. One proposed mechanism for this disparity has been attributed to alternative splicing that produces protein an isoform that serves a particular purpose in the photoreceptor sensory cilium and is unnecessary/or not present for other cell types. BBS8 is a potential example of this phenomenon, in which specific mutations result in non-syndromic RP, while other mutations result in BBS (Murphy et al. 2015). The mechanisms underlying the specific phenotype associated with photoreceptor cells is important to understand to elucidate the processes that result in specialization of this primary cilium.

\section{APPROACHES AND DIRECTIONS FOR FUTURE RESEARCH}

Photoreceptor research has been a slow process as a result of the difficulties associated with primarily studying animal models because of the lack of a heterologous tissue system. Most studies of underlying cellular pathways involve basic in vitro experiments determining protein:protein interactions and localization that must be correlated with clinical manifestations 
and phenotypes of animal models. Additionally, these specialized cilia require a high level of spatiotemporal regulation of protein trafficking to maintain very distinct compartments. Small GTPases are no doubt crucial to this regulation and it is no wonder that an increasing number of these molecular switches have been implicated in blinding disease states with photoreceptor cell dysfunction. The study of small GTPases is fairly straightforward, as generally the mechanisms of these proteins are conserved from cell to cell with the primary differences being the effector proteins. Although these processes are often complex, involving many proteins for each step, the modes in which the small GTPase can be manipulated in vitro and in vivo allow for a stepwise process for analysis of the specific protein of interest. Nevertheless, while there has been significant breakthroughs in recent years, small GTPases remain understudied in the realm of photoreceptor cell biology.

The first step in determining the extent to which small GTPases play a role in photoreceptor cell function is to obtain a comprehensive list of the pertinent small GTPases. Previous studies have attempted to isolate the OS or other compartments in order to identify the proteins by mass spectrometry (Liu et al. 2007). However, there are difficulties with this approach. One major problem is that it may not capture the proteins involved in ciliary development or trafficking because those proteins only function there transiently and do not necessarily maintain localization there. For example, small GTPases can possibly be localized to all compartments, but only function at the cilium or OS. ARL3 is a great example of this as it is found throughout the photoreceptor cell, but appears to function (at least for prenylated protein trafficking) in the OS or connecting cilium (Z. C. Wright et al. 2016). Nonetheless, these proteomic libraries are a great place to start looking for photoreceptor developmental and trafficking related phenotypes associated with manipulation of candidate proteins. Additionally, 
candidate small GTPases for photoreceptor function can be identified by surveying literature focusing on other ciliated cell types with known analogous functions or by screens of candidate proteins in RPE cell lines as done previously (Yoshimura et al. 2007). Screening the Arl subfamily of small GTPases for ciliary developmental effects similar to Yoshimura et al. (2007) would allow provide direction for future research. Screening systems could be designed to test specific functional effects resulting from the loss or overexpression of a set of proteins. For example, a heterologous high-throughput screening system for identifying small GTPases crucial for trafficking of specific photoreceptor proteins would accelerate research dramatically. A system like this could utilize known proteins that are localized to cilia in RPE cells for example, and manipulate expression of candidate small GTPases in that system.

The currently known small GTPases involved in photoreceptor OS biogenesis and trafficking are understudied and often have confusing or contradictory data. To extend our understanding of these proteins, we must determine the regulators and effectors of the proteins and their functions in the photoreceptor cell environment. Simply identifying the GEF or GAP of a small GTPase has a great impact on the direction of research and the underlying proposed mechanisms of action. For example, the recently identified GEF of ARL3, ARL13b, has helped build a clearer mechanism of prenylated phototransduction proteins in photoreceptor cells (Gotthardt et al. 2015). Because ARL13b has been found to be localized to the OS and connecting cilium, it confirms the expected function of active ARL3 which is to release prenylated cargo from their respective prenyl transferase (e.g. PDE6 and $\operatorname{PrBP} \delta$ ) in the proper location, i.e. connecting cilium. However, these data are limited in that they are typically obtained in vitro and not in photoreceptor cells. It is possible that the proteins that can act as a GEF do not do so in vivo and that the GEF in photoreceptor cells is yet to be identified. 
Additionally, considering the diverse roles that these proteins can play it is possible that they have multiple GEF proteins that distinguish the molecular cascade in which they are involved. To address this, mouse models targeting function of the GEF protein are necessary to provide insight into the effectors function and corroborate previous results.

Determining the function of a given small GTPase has major complications as well. First, conditional approaches are often necessary as complete knockouts confer embryonic lethality. More importantly, another major difficulty is that these proteins can serve multiple functions in various processes at different timepoints during development of a specific cell type. For example, ARL3 appears to have multiple functions in photoreceptor cells, nuclear migration, protein trafficking, and OS development (Z. C. Wright et al. 2016; Hanke-Gogokhia et al. 2016). This is an important case because it demonstrates the need to study effects of small GTPase dysfunction by manipulating it at different timepoints in photoreceptor development with various in vivo techniques. This can be done by utilizing alternative promoters to drive transgenic expression of Cre recombinase (for conditional gene knockout) or dominant active/inactive mutants. Considering the advent of Crispr-Cas9 genome editing technology, we are able to generate new animal models quicker and more cost effectively. With that in mind, studying the loss or mutation of these proteins using tamoxifen-inducible conditional mouse models is ideal and will provide great insight into their molecular function. Another complication associated with overexpression of dominant active mutants is off-target effects. It is possible that these mutants will function in molecular pathways that the endogenous protein does not because normally they will only be activated by a cognate GEF that is localized to only a specific compartment. 
With these complications in mind, it is undoubtedly necessary to use a combinatorial approach to study small GTPases. Furthermore, expanding the methods through which we study photoreceptor proteins will be crucial in the future for unveiling molecular functions specific to this cell type. More specifically, the development of a heterologous system such as an ex vivo tissue culture in which ciliary dynamics and protein trafficking can be analyzed in live cells will provide breakthroughs in the field. Exploring the role of small GTPases in photoreceptor cells will provide insights into the molecular events that give rise to the OS and allow proteins to be specifically trafficked to there. This research will be crucial in understanding the pathogenesis underlying retinal disease states and allow for the identification of therapeutic targets. 


\section{REFERENCES}

Apolloni, a, I a Prior, M Lindsay, R G Parton, and J F Hancock. 2000. "H-Ras but Not K-Ras Traffics to the Plasma Membrane through the Exocytic Pathway.” Molecular and Cellular Biology 20 (7): 2475-87. doi:10.1128/MCB.20.7.2475-2487.2000.

Avidor-Reiss, Tomer, Andreia M Maer, Edmund Koundakjian, Andrey Polyanovsky, Thomas Keil, Shankar Subramaniam, and Charles S Zuker. 2004. "Decoding Cilia Function.” Cell 117 (4): 527-39. doi:10.1016/S0092-8674(04)00412-X.

Bartolini, Francesca, Arunashree Bhamidipati, Scott Thomas, Uwe Schwahn, Sally a. Lewis, and Nicholas J. Cowan. 2002. “Functional Overlap between Retinitis Pigmentosa 2 Protein and the Tubulin-Specific Chaperone Cofactor C.” Journal of Biological Chemistry 277 (17): 14629-34. doi:10.1074/jbc.M200128200.

Blacque, Oliver E., Michael J. Reardon, Chunmei Li, Jonathan McCarthy, Moe R. Mahjoub, Stephen J. Ansley, Jose L. Badano, et al. 2004. "Loss of C. Elegans BBS-7 and BBS-8 Protein Function Results in Cilia Defects and Compromised Intraflagellar Transport.” Genes and Development 18 (13): 1630-42. doi:10.1101/gad.1194004.

Boehlke, Christopher, Mikhail Bashkurov, Andrea Buescher, Theda Krick, Anne-Katharina John, Roland Nitschke, Gerd Walz, and E Wolfgang Kuehn. 2010. "Differential Role of Rab Proteins in Ciliary Trafficking: Rab23 Regulates Smoothened Levels.” Journal of Cell Science 123 (Pt 9): 1460-67. doi:10.1242/jcs.058883.

Branham, Kari, Mohammad Othman, Matthew Brumm, Athanasios J. Karoukis, Pelin AtmacaSonmez, Beverly M. Yashar, Sharon B. Schwartz, et al. 2012. "Mutations in RPGR and RP2 Account for 15\% of Males with Simplex Retinal Degenerative Disease.” Investigative 
Ophthalmology and Visual Science 53 (13): 8232-37. doi:10.1167/iovs.12-11025.

Burd, Christopher G., Todd I. Strochlic, and Subba R. Gangi Setty. 2004. “Arf-like GTPases:

Not so Arf-like after All.” Trends in Cell Biology 14 (12): 687-94.

doi:10.1016/j.tcb.2004.10.004.

Cantagrel, Vincent, Jennifer L. Silhavy, Stephanie L. Bielas, Dominika Swistun, Sarah E. Marsh, Julien Y. Bertrand, Sophie Audollent, et al. 2008. "Mutations in the Cilia Gene ARL13B Lead to the Classical Form of Joubert Syndrome.” American Journal of Human Genetics 83 (2): 170-79. doi:10.1016/j.ajhg.2008.06.023.

Caspary, Tamara, Christine E. Larkins, and Kathryn V. Anderson. 2007. “The Graded Response to Sonic Hedgehog Depends on Cilia Architecture.” Developmental Cell 12 (5): 767-78. doi:10.1016/j.devcel.2007.03.004.

Chandra, Anchal, Hernán E. Grecco, Venkat Pisupati, David Perera, Liam Cassidy, Ferdinandos Skoulidis, Shehab a. Ismail, et al. 2012. “The GDI-like Solubilizing Factor PDE $\delta$ Sustains the Spatial Organization and Signalling of Ras Family Proteins.” Nature Cell Biology 14 (3). Nature Publishing Group: 329-329. doi:10.1038/ncb2462.

Chen, Keng, Chwee Tat Koe, Zhanyuan Benny Xing, Xiaolin Tian, Fabrizio Rossi, Cheng Wang, Quan Tang, et al. 2016. “Arl2- and Msps-Dependent Microtubule Growth Governs Asymmetric Division.” The Journal of Cell Biology 212 (6): jcb.201503047. doi:10.1083/jcb.201503047.

Cherfils, Jacqueline, and Mahel Zeghouf. 2013. “Regulation of Small GTPases by GEFs, GAPs, and GDIs.” Physiological Reviews 93 (1): 269-309. doi:10.1152/physrev.00003.2012. 
Chiang, Annie P, Darryl Nishimura, Charles Searby, Khalil Elbedour, Rivka Carmi, Amanda L Ferguson, Jenifer Secrist, et al. 2004. “Comparative Genomic Analysis Identifies an ADPRibosylation Factor-like Gene as the Cause of Bardet-Biedl Syndrome (BBS3).” American Journal of Human Genetics 75 (3): 475-84. doi:10.1086/423903.

Datta, Poppy, Chantal Allamargot, Joseph S. Hudson, Emily K. Andersen, Sajag Bhattarai, Arlene V. Drack, Val C. Sheffield, and Seongjin Seo. 2015. “Accumulation of Non-Outer Segment Proteins in the Outer Segment Underlies Photoreceptor Degeneration in BardetBiedl Syndrome.” Proceedings of the National Academy of Sciences 112 (32): E4400-4409. doi:10.1073/pnas.1510111112.

Davidson, Alice E., Nele Schwarz, Lina Zelinger, Gabriele Stern-Schneider, Amelia Shoemark, Benjamin Spitzbarth, Menachem Gross, et al. 2013. "Mutations in ARL2BP, Encoding ADP-Ribosylation-Factor-like 2 Binding Protein, Cause Autosomal-Recessive Retinitis Pigmentosa." American Journal of Human Genetics 93 (2). The American Society of Human Genetics: 321-29. doi:10.1016/j.ajhg.2013.06.003.

Deretic, D, L a Huber, N Ransom, M Mancini, K Simons, and D S Papermaster. 1995. "Rab8 in Retinal Photoreceptors May Participate in Rhodopsin Transport and in Rod Outer Segment Disk Morphogenesis.” Journal of Cell Science 108 ( Pt 1: 215-24.

Deretic, Dusanka. 2013. “Crosstalk of Arf and Rab GTPases En Route to Cilia.” Small GTPases 4 (2): 70-77. doi:10.4161/sgtp.24396.

Deretic, Dusanka, and Jing Wang. 2012. “Molecular Assemblies That Control Rhodopsin Transport to the Cilia.” Vision Research 75: 5-10. doi:10.1016/j.visres.2012.07.015.

Deretic, Dusanka, Andrew H Williams, Nancy Ransom, Valerie Morel, Paul A Hargrave, and 
Anatol Arendt. 2005. "Rhodopsin C Terminus, the Site of Mutations Causing Retinal Disease, Regulates Trafficking by Binding to ADP-Ribosylation Factor 4 (ARF4).” Proceedings of the National Academy of Sciences of the United States of America 102 (9): 3301-6. doi:10.1073/pnas.0500095102.

Evans, R. Jane, Nele Schwarz, Kerstin Nagel-Wolfrum, Uwe Wolfrum, Alison J. Hardcastle, and Michael E. Cheetham. 2010. “The Retinitis Pigmentosa Protein RP2 Links Pericentriolar Vesicle Transport between the Golgi and the Primary Cilium.” Human Molecular Genetics 19 (7): 1358-67. doi:10.1093/hmg/ddq012.

Fan, Yanli, Muneer a Esmail, Stephen J Ansley, Oliver E Blacque, Keith Boroevich, Alison J Ross, Susan J Moore, et al. 2004. "Mutations in a Member of the Ras Superfamily of Small GTP-Binding Proteins Causes Bardet-Biedl Syndrome.” Nature Genetics 36 (9): 989-93. doi:10.1038/ng1414.

Geng, Lin, Dayne Okuhara, Zhiheng Yu, Xin Tian, Yiqiang Cai, Sekiya Shibazaki, and Stefan Somlo. 2006. "Polycystin-2 Traffics to Cilia Independently of Polycystin-1 by Using an NTerminal RVxP Motif.” Journal of Cell Science 119 (Pt 7): 1383-95. doi:10.1242/jcs.02818.

Gillingham, Alison K, and Sean Munro. 2007. "The Small G Proteins of the Arf Family and Their Regulators.” Annual Review of Cell and Developmental Biology 23: 579-611. doi:10.1146/annurev.cellbio.23.090506.123209.

Gotthardt, Katja, Mandy Lokaj, Carolin Koerner, Nathalie Falk, Andreas Gießl, and Alfred Wittinghofer. 2015. “A G-Protein Activation Cascade from Arl13B to Arl3 and Implications for Ciliary Targeting of Lipidated Proteins.” Edited by David E Clapham. 
eLife.

Grayson, Celene, Francesca Bartolini, J Paul Chapple, Keith R Willison, Arunashree

Bhamidipati, Sally a Lewis, Philip J Luthert, Alison J Hardcastle, Nicholas J Cowan, and Michael E Cheetham. 2002. "Localization in the Human Retina of the X-Linked Retinitis Pigmentosa Protein RP2, Its Homologue Cofactor C and the RP2 Interacting Protein Arl3.” Human Molecular Genetics 11 (24): 3065-74.

Hanke-Gogokhia, Christin, Zhijian Wu, Cecilia D. Gerstner, Jeanne M. Frederick, Houbin Zhang, and Wolfgang Baehr. 2016. “Arf-like Protein 3 (ARL3) Regulates Protein Trafficking and Ciliogenesis in Mouse Photoreceptors.” Journal of Biological Chemistry 3: jbc.M115.710954. doi:10.1074/jbc.M115.710954.

Hattula, Katarina, Johanna Furuhjelm, Airi Arffman, and Johan Peränen. 2002. “A Rab8Specific GDP/GTP Exchange Factor Is Involved in Actin Remodeling and Polarized Membrane Transport.” Molecular Biology of the Cell 13 (September): 3268-80. doi:10.1091/mbc.E02.

Hong, Dong Hyun, Guohua Yue, Michael Adamian, and Tiansen Li. 2001. "Retinitis Pigmentosa GTPase Regulator (RPGR)-Interacting Protein Is Stably Associated with the Photoreceptor Ciliary Axoneme and Anchors RPGR to the Connecting Cilium.” Journal of Biological Chemistry 276 (15): 12091-99. doi:10.1074/jbc.M009351200.

Iannaccone, Alessandro, David Man, Naushin Waseem, Barbara J. Jennings, Madhavi Ganapathiraju, Kevin Gallaher, Elisheva Reese, Shomi S. Bhattacharya, and Judith KleinSeetharaman. 2006. "Retinitis Pigmentosa Associated with Rhodopsin Mutations: Correlation between Phenotypic Variability and Molecular Effects.” Vision Research 46 
(27): 4556-67. doi:10.1016/j.visres.2006.08.018.

Ismail, Shehab a, Yong-Xiang Chen, Mandy Miertzschke, Ingrid R Vetter, Carolin Koerner, and Alfred Wittinghofer. 2012. "Structural Basis for Arl3-Specific Release of Myristoylated Ciliary Cargo from UNC119.” The EMBO Journal 31 (20). Nature Publishing Group: 4085-94. doi:10.1038/emboj.2012.257.

Ismail, Shehab a, Yong-Xiang Chen, Alexandra Rusinova, Anchal Chandra, Martin Bierbaum, Lothar Gremer, Gemma Triola, Herbert Waldmann, Philippe I H Bastiaens, and Alfred Wittinghofer. 2011. “Arl2-GTP and Arl3-GTP Regulate a GDI-like Transport System for Farnesylated Cargo.” Nature Chemical Biology 7 (12): 942-49. doi:10.1038/nchembio.686.

Jin, Hua, Susan Roehl White, Toshinobu Shida, Stefan Schulz, Mike Aguiar, Steven P. Gygi, J. Fernando Bazan, and Maxence V. Nachury. 2010. "The Conserved Bardet-Biedl Syndrome Proteins Assemble a Coat That Traffics Membrane Proteins to Cilia.” Cell 141 (7): 120819. doi:10.1016/j.cell.2010.05.015.

Jin, Mingyue, Masami Yamada, Yoshiyuki Arai, Takeharu Nagai, and Shinji Hirotsune. 2014. “Arl3 and LC8 Regulate Dissociation of Dynactin from Dynein.” Nature Communications 5. Nature Publishing Group: 5295. doi:10.1038/ncomms6295.

Kim, Hyunho, Hangxue Xu, Qin Yao, Weizhe Li, Qiong Huang, Patricia Outeda, Valeriu Cebotaru, et al. 2014. "Ciliary Membrane Proteins Traffic through the Golgi via a Rabep1/GGA1/Arl3-Dependent Mechanism.” Nature Communications 5 (May). Nature Publishing Group: 5482. doi:10.1038/ncomms6482.

Knödler, Andreas, Shanshan Feng, Jian Zhang, Xiaoyu Zhang, Amlan Das, Johan Peränen, and Wei Guo. 2010. “Coordination of Rab8 and Rab11 in Primary Ciliogenesis.” Proceedings 
of the National Academy of Sciences of the United States of America 107 (14): 6346-51. doi:10.1073/pnas.1002401107.

Kühnel, Karin, Stefan Veltel, Ilme Schlichting, and Alfred Wittinghofer. 2006. “Crystal Structure of the Human Retinitis Pigmentosa 2 Protein and Its Interaction with Arl3.” Structure 14 (2): 367-78. doi:10.1016/j.str.2005.11.008.

Lai, Cary K, Nidhi Gupta, Xiaohui Wen, Linda Rangell, Ben Chih, Andrew S Peterson, J Fernando Bazan, Li Li, and Suzie J Scales. 2011. "Functional Characterization of Putative Cilia Genes by High-Content Analysis.” Molecular Biology of the Cell 22 (7): 1104-19. doi:10.1091/mbc.E10-07-0596.

Li, Linjing, Naheed Khan, Toby Hurd, Amiya Kumar Ghosh, Christiana Cheng, Robert Molday, John R. Heckenlively, Anand Swaroop, and Hemant Khanna. 2013. “Ablation of the XLinked Retinitis Pigmentosa 2 (Rp2) Gene in Mice Results in Opsin Mislocalization and Photoreceptor Degeneration.” Investigative Ophthalmology and Visual Science 54 (7): 4503-11. doi:10.1167/iovs.13-12140.

Li, Yujie, and Jinghua Hu. 2011. “Small GTPases and Cilia.” Protein Cell 2 (1): 13-25. doi:10.1016/j.biotechadv.2011.08.021.Secreted.

Li, Yujie, Qing Wei, Yuxia Zhang, Kun Ling, and Jinghua Hu. 2010. “The Small GTPases ARL13 and ARL-3 Coordinate Intraflagellar Transport and Ciliogenesis.” Journal of Cell Biology 189 (6): 1039-51. doi:10.1083/jcb.200912001.

Liu, Qin, Glenn Tan, Natasha Levenkova, Tiansen Li, Edward N Pugh, John J Rux, David W Speicher, and Eric a Pierce. 2007. “The Proteome of the Mouse Photoreceptor Sensory Cilium Complex.” Molecular \& Cellular Proteomics : MCP 6 (8): 1299-1317. 
doi:10.1074/mcp.M700054-MCP200.

Loktev, Alexander V., Qihong Zhang, John S. Beck, Charles C. Searby, Todd E. Scheetz, J. Fernando Bazan, Diane C. Slusarski, Val C. Sheffield, Peter K. Jackson, and Maxence V. Nachury. 2008. “A BBSome Subunit Links Ciliogenesis, Microtubule Stability, and Acetylation.” Developmental Cell 15 (6): 854-65. doi:10.1016/j.devcel.2008.11.001.

Mazelova, Jana, Lisa Astuto-Gribble, Hiroki Inoue, Beatrice M Tam, Eric Schonteich, Rytis Prekeris, Orson L Moritz, Paul a Randazzo, and Dusanka Deretic. 2009. “Ciliary Targeting Motif VxPx Directs Assembly of a Trafficking Module through Arf4.” The EMBO Journal 28 (3): 183-92. doi:10.1038/emboj.2008.267.

Miertzschke, Mandy, Carolin Koerner, Michael Spoerner, and Alfred Wittinghofer. 2014. "Structural Insights into the Small G-Protein Arl13B and Implications for Joubert Syndrome.” Biochem. J 457: 301-11. doi:10.1042/BJ20131097.

Mori, Risa, and Takashi Toda. 2013. “The Dual Role of Fission Yeast Tbc1/cofactor C Orchestrates Microtubule Homeostasis in Tubulin Folding and as a GAP for GTPase Alp41/Arl2.” Molecular Biology of the Cell 24: 1-29. doi:10.1091/mbc.E12-11-0792.

Moritz, O L, B M Tam, L L Hurd, J Peränen, D Deretic, and D S Papermaster. 2001. “Mutant rab8 Impairs Docking and Fusion of Rhodopsin-Bearing Post-Golgi Membranes and Causes Cell Death of Transgenic Xenopus Rods.” Molecular Biology of the Cell 12 (8): 2341-51.

Murga-Zamalloa, Carlos A., Stephen J. Atkins, Johan Peranen, Anand Swaroop, and Hemant Khanna. 2010. "Interaction of Retinitis Pigmentosa GTPase Regulator (RPGR) with RAB8A GTPase: Implications for Cilia Dysfunction and Photoreceptor Degeneration.” Human Molecular Genetics 19 (18): 3591-98. doi:10.1093/hmg/ddq275. 
Murphy, Daniel, Ratnesh Singh, Saravanan Kolandaivelu, Visvanathan Ramamurthy, and Peter Stoilov. 2015. “Alternative Splicing Shapes the Phenotype of a Mutation in BBS8 To Cause Nonsyndromic Retinitis Pigmentosa.” Molecular and Cellular Biology 35 (10): 1860-70. doi:10.1128/MCB.00040-15.

Murray, Anne R, Steven J Fliesler, and Muayyad R Al-Ubaidi. 2009. "Rhodopsin: The Functional Significance of Asn-Linked Glycosylation and Other Post-Translational Modifications.” Ophthalmic Genetics 30 (3): 109-20. doi:10.1080/13816810902962405.

Nachury, Maxence V., Alexander V. Loktev, Qihong Zhang, Christopher J. Westlake, Johan Per??nen, Andreas Merdes, Diane C. Slusarski, et al. 2007. “A Core Complex of BBS Proteins Cooperates with the GTPase Rab8 to Promote Ciliary Membrane Biogenesis.” Cell 129 (6): 1201-13. doi:10.1016/j.cell.2007.03.053.

Nachury, Maxence V, E Scott Seeley, and Hua Jin. 2010. “Trafficking to the Ciliary Membrane: How to Get across the Periciliary Diffusion Barrier?” Annual Review of Cell and Developmental Biology 26: 59-87. doi:10.1146/annurev.cellbio.042308.113337.

Nithiananatham, Stanley, Sinh Le, Elbert Seto, Weitao Jia, Julie Leary, Kevin D Corbett, and K Jeffrey. 2015. “Tubulin Cofactors and Arl2 Are Cage-like Chaperones That Regulate the Soluble A $\beta$-Tubulin Pool for Microtubule Dynamics.” eLife, no. July: 1-33. doi:10.7554/eLife.08811.

Pearring, Jillian N., Raquel Y. Salinas, Sheila a. Baker, and Vadim Y. Arshavsky. 2013. "Protein Sorting, Targeting and Trafficking in Photoreceptor Cells.” Progress in Retinal and Eye Research 36. Elsevier Ltd: 24-51. doi:10.1016/j.preteyeres.2013.03.002.

Petrs-Silva, Hilda, and Rafael Linden. 2013. “Advances in Gene Therapy Technologies to Treat 
Retinitis Pigmentosa.” Clinical Ophthalmology 8: 127-36. doi:10.2147/OPTH.S38041.

Riveiro-Álvarez, Rosa, Yajing (Angela) Xie, Miguel-Ángel López-Martínez, Tomasz Gambin, Raquel Pérez-Carro, Almudena Ávila-Fernández, María-Isabel López-Molina, et al. 2015. "New Mutations in Theln RAB28\n Gene in 2 Spanish Families With Cone-Rod Dystrophy.” JAMA Ophthalmology 133 (2): 133. doi:10.1001/jamaophthalmol.2014.4266.

Roosing, Susanne, Rob W J Collin, Anneke I den Hollander, Frans P M Cremers, and Anna M Siemiatkowska. 2014. "Prenylation Defects in Inherited Retinal Diseases.” Journal of Medical Genetics 51 (3): 143-51. doi:10.1136/jmedgenet-2013-102138.

Roosing, Susanne, Klaus Rohrschneider, Avigail Beryozkin, Dror Sharon, Nicole Weisschuh, Jennifer Staller, Susanne Kohl, et al. 2013. “Mutations in RAB28, Encoding a Farnesylated Small Gtpase, Are Associated with Autosomal-Recessive Cone-Rod Dystrophy.” American Journal of Human Genetics 93 (1). The American Society of Human Genetics: 110-17. doi:10.1016/j.ajhg.2013.05.005.

Rossmiller, Brian, Haoyu Mao, and Alfred S Lewin. 2012. “Gene Therapy in Animal Models of Autosomal Dominant Retinitis Pigmentosa.” Molecular Vision 18 (February): 2479-96. http://www.pubmedcentral.nih.gov/articlerender.fcgi?artid=3472929\&tool=pmcentrez\&ren dertype=abstract.

Schrick, Jeffrey J, Peter Vogel, Alejandro Abuin, Billy Hampton, and Dennis S Rice. 2006. “ADP-Ribosylation Factor-like 3 Is Involved in Kidney and Photoreceptor Development.” The American Journal of Pathology 168 (4): 1288-98. doi:10.2353/ajpath.2006.050941.

Schwarz, Nele, Tatiana V. Novoselova, Robin Wait, Alison J. Hardcastle, and Michael E. Cheetham. 2012. “The X-Linked Retinitis Pigmentosa Protein RP2 Facilitates G Protein 
Traffic.” Human Molecular Genetics 21 (4): 863-73. doi:10.1093/hmg/ddr520.

Tian, Guoling, Simi Thomas, and Nicholas J. Cowan. 2010. “Effect of TBCD and Its Regulatory Interactor Arl2 on Tubulin and Microtubule Integrity.” Cytoskeleton 67 (11): 706-14. doi:10.1002/cm.20480.

Veltel, Stefan, Aleksandra Kravchenko, Shehab Ismail, and Alfred Wittinghofer. 2008. "Specificity of Arl2/Arl3 Signaling Is Mediated by a Ternary Arl3-Effector-GAP Complex.” FEBS Letters 582 (17): 2501-7. doi:10.1016/j.febslet.2008.05.053.

Wang, D Y, W M Chan, P O S Tam, L Baum, D S C Lam, K K L Chong, B J Fan, and C P Pang. 2005. "Gene Mutations in Retinitis Pigmentosa and Their Clinical Implications.” Clinica Chimica Acta; International Journal of Clinical Chemistry 351 (1-2): 5-16. doi:10.1016/j.cccn.2004.08.004.

Wang, Jing, Yoshiko Morita, Jana Mazelova, and Dusanka Deretic. 2012. "The Arf GAP ASAP1 Provides a Platform to Regulate Arf4- and Rab11-Rab8-Mediated Ciliary Receptor Targeting.” The EMBO Journal 31 (20). Nature Publishing Group: 4057-71. doi:10.1038/emboj.2012.253.

Ward, Heather H, Ursa Brown-Glaberman, Jing Wang, Yoshiko Morita, Seth L Alper, Edward J Bedrick, Vincent H Gattone, Dusanka Deretic, and Angela Wandinger-Ness. 2011. “A Conserved Signal and GTPase Complex Are Required for the Ciliary Transport of Polycystin-1.” Molecular Biology of the Cell 22 (18): 3289-3305. doi:10.1091/mbc.E11-010082.

Wiens, Cheryl J., Yufeng Tong, Muneer a. Esmail, Edwin Oh, Jantje M. Gerdes, Jihong Wang, Wolfram Tempel, et al. 2010. "Bardet-Biedl Syndrome-Associated Small GTPase ARL6 
(BBS3) Functions at or near the Ciliary Gate and Modulates Wnt Signaling.” Journal of Biological Chemistry 285 (21): 16218-30. doi:10.1074/jbc.M109.070953.

Wright, Kevin J., Lisa M. Baye, Anique Olivier-Mason, Saikat Mukhopadhyay, Liyun Sang, Mandy Kwong, Weiru Wang, et al. 2011. “An ARL3-UNC119-RP2 GTPase Cycle Targets Myristoylated NPHP3 to the Primary Cilium.” Genes and Development 25 (22): 2347-60. doi:10.1101/gad.173443.111.

Wright, Zachary C, Ratnesh K Singh, Ryan Alpino, Andrew F X Goldberg, Maxim Sokolov, and Visvanathan Ramamurthy. 2016. “ARL3 Regulates Trafficking of Prenylated Phototransduction Proteins to the Rod Outer Segment.” Human Molecular Genetics , no. March (March): 1-46. doi:10.1093/hmg/ddw077.

Yoshimura, Shin Ichiro, Johannes Egerer, Evelyn Fuchs, Alexander K. Haas, and Francis A. Barr. 2007. “Functional Dissection of Rab GTPases Involved in Primary Cilium Formation.” Journal of Cell Biology 178 (3): 363-69. doi:10.1083/jcb.200703047.

Zhang, H, S Li, T Doan, F Rieke, P B Detwiler, J M Frederick, and W Baehr. 2007. "Deletion of PrBP/delta Impedes Transport of GRK1 and PDE6 Catalytic Subunits to Photoreceptor Outer Segments." Proceedings of the National Academy of Sciences of the United States of America 104 (21): 8857-62. doi:10.1073/pnas.0701681104.

Zhang, Houbin, Ryan Constantine, Jeanne M. Frederick, and Wolfgang Baehr. 2012. “The Prenyl-Binding Protein PrBP/ $\delta$ : A Chaperone Participating in Intracellular Trafficking.” Vision Research 75. Elsevier Ltd: 19-25. doi:10.1016/j.visres.2012.08.013.

Zhang, Qihong, Darryl Nishimura, Seongjin Seo, Tim Vogel, Donald a Morgan, and Charles Searby. 2011. "Reveals Common BBS-Associated Phenotypes and Bbs3 Unique 
Phenotypes." Proceedings of the National Academy of Sciences of the United States of America 3 (51): 20678-83. doi:10.1073/pnas.1113220108//DCSupplemental.www.pnas.org/cgi/doi/10.1073/pnas.1113220108.

Zhang, Qihong, Darryl Nishimura, Seongjin Seo, Tim Vogel, Donald A Morgan, Charles Searby, Kevin Bugge, Edwin M Stone, Kamal Rahmouni, and Val C Sheffield. 2011. “Bardet-Biedl Syndrome 3 (Bbs3) Knockout Mouse Model Reveals Common BBS-Associated Phenotypes and Bbs3 Unique Phenotypes.” Proceedings of the National Academy of Sciences of the United States of America 108 (51): 20678-83. doi:10.1073/pnas.1113220108.

Zhang, Qihong, Seongjin Seo, Kevin Bugge, Edwin M. Stone, and Val C. Sheffield. 2012. "BBS Proteins Interact Genetically with the IFT Pathway to Influence SHH-Related Phenotypes.” Human Molecular Genetics 21 (9): 1945-53. doi:10.1093/hmg/dds004.

Zhou, Cheng-jing, Leslie Cunningham, Adam Marcus, Yawei Li, and Richard A. Kahn. 2006. “Arl2 and Arl3 Regulate Different Microtubule-Dependent Processes.” Molecular Biology of the Cell 17 (5): 2476-87. doi:10.1091/mbc.E05. 


\section{Chapter 2:}

ARL3 regulates trafficking of prenylated phototransduction proteins to the rod outer segment.

Zachary C. Wright ${ }^{1 a}$, Ratnesh K. Singh ${ }^{1 a}$, Ryan Alpino ${ }^{1 a}$, Andrew F.X. Goldberg², Maxim Sokolov ${ }^{1 a, b, c}$, and Visvanathan Ramamurthy ${ }^{1 a, b, c *}$

Departments of ${ }^{\mathrm{a}}$ Ophthalmology and ${ }^{\mathrm{b}}$ Biochemistry, ${ }^{\mathrm{c}}$ Center for Neuroscience, Robert C. Byrd Health Sciences Center, ${ }^{1}$ West Virginia University, Morgantown, West Virginia 26506, USA;

${ }^{2}$ Eye Research Institute, Oakland University, Rochester, Michigan, 48309, USA

*Address Correspondence to: Visvanathan Ramamurthy, West Virginia University Eye Institute, One Medical Drive, E-363, Morgantown, WV 26506-9193, Tel: 304598 6940; Fax: 304-5986928; Email: ramamurthyv@wvumedicine.org 


\section{ABSTRACT}

The small GTPase, ADP-ribosylation factor-like 3 (ARL3), has been proposed to participate in the transport of proteins in photoreceptor cells. Moreover, it has been implicated in the pathogenesis associated with X-linked retinitis pigmentosa (XLRP) resulting from mutations in the ARL3 GTPase activating protein, retinitis pigmentosa 2 (RP2). To determine the importance of ARL3 in rod photoreceptor cells, we generated transgenic mice expressing a dominant active form of ARL3 (ARL3-Q71L) under a rod-specific promoter. ARL3-Q71L animals exhibited extensive rod cell death after post-natal day 30 (PN30) and degeneration was complete by PN70. Prior to the onset of cell death, rod photoresponse was significantly reduced along with a robust decrease in rod phosphodiesterase 6 (PDE6) and G-protein receptor kinase-1 (GRK1) levels. Furthermore, assembled phosphodiesterase-6 (PDE6) subunits, rod transducin, and G-protein receptor kinase-1 (GRK1) accumulated on large punctate structures within the inner segment in ARL3-Q71L retina. Defective trafficking of prenylated proteins is likely due to sequestration of prenyl binding protein $\delta(\operatorname{PrBP} \delta$ ) by ARL3-Q71L as we demonstrate a specific interaction between these proteins in the retina. Unexpectedly, our studies also revealed a novel role for ARL3 in the migration of photoreceptor nuclei. In conclusion, this study identifies ARL3 as a key player in prenylated protein trafficking in rod photoreceptor cells and establishes the potential role for ARL3 dysregulation in the pathogenesis of RP2-related forms of XLRP. 


\section{INTRODUCTION}

Trafficking of proteins to the right destination is crucial for proper functioning of a cell. The significance of this process is exemplified by polarized photoreceptor cells in the retina. In photoreceptors, proteins are synthesized in the inner segment (IS) and are selectively transported to their site of action in the outer segment (OS). In addition, the high rate of protein turnover resulting from the renewal of the OS requires an efficient mode of protein movement between various compartments in photoreceptor cells. Defective trafficking is a known cause of blinding diseases such as retinitis pigmentosa in humans $(1,2)$. Despite the importance of efficient trafficking of proteins in photoreceptor cells, the basic mechanism behind the regulation and polarized movement of OS proteins are poorly understood.

Small GTPases are molecular switches cycling between GTP bound “on” and GDP bound "off” states and are known regulators of vesicular trafficking, aiding in the movement of proteins and lipids between different cellular compartments. ARL3, a member of the ADPribosylation factor (ARF) family, is one such GTPase that is important for photoreceptor development. Absence of ARL3 in a mouse knockout leads to poor development of photoreceptor cell OS and rapid degeneration of photoreceptor neurons (3). However, little is known about the role of ARL3 in vivo (4). ARL3 was identified as an effector protein of retinitis pigmentosa protein 2 (RP2). RP2 acts as a GTPase activating protein (GAP) thereby facilitating conversion of active ARL3-GTP to inactive ARL3-GDP, accelerating the intrinsic GTPase activity up to 90000-fold (5). Defects in the RP2 gene result in an X-linked form of retinitis pigmentosa (XLRP), a very severe form of inherited blindness in males. Mutations in this gene account for approximately 7-18\% of these XLRP cases (6). Disruption of the RP2 gene likely leads to dysregulation of its interacting protein ARL3, maintaining it in an active, GTP-bound 
state as it would rely solely on its slow intrinsic rate $\left(\mathrm{k}_{\text {cat }}\right.$ of $\left.0.007 / \mathrm{min}\right)(5,7,8)$. Additionally, ARL3 has been implicated in the transport of lipid-modified proteins through its association with the lipid-binding proteins, prenyl binding protein $\delta(\operatorname{PrBP} \delta$; also known as PDE6 $\delta)$ and the $\operatorname{PrBP} \delta$ homolog Unc119 (also known as HRG4) (9-11). PrBP $\delta$ has a lipid binding pocket that is exposed in the "open” conformation and inaccessible in the "closed" conformation. Active ARL3-GTP (but not the inactive ARL3-GDP) binds to an allosteric site that induces the change of $\operatorname{PrBP} \delta$ to the "closed" confirmation thereby lowering affinity for the prenyl group on an effector protein. These in vitro data suggest that ARL3 acts as a release factor for prenylated cargo being carried by $\operatorname{PrBP} \delta(10)$. Related studies have shown that loss of $\operatorname{PrBP} \delta$ in mice lead to defective trafficking of prenylated proteins to rod and cone outer segments (12). More recently, it has been shown that RP2 knockout mice exhibit slow progressive retinal degeneration $(7,13)$ associated with mislocalization of prenylated phosphodiesterase 6 (PDE6) and G-protein receptor kinase 1 (GRK1) (7). This result has also been recapitulated in RP2 null zebrafish, which showed slow degeneration and mislocalization of prenylated proteins (14). Absence of RP2 likely leads to overactive ARL3 as the GAP activity is lost. We believe that ARL3 overactivity is the intermediary that causes the phenotype observed in RP2 mouse models and associated human disease.

The purpose of the present study is to gain insight into the role of ARL3 in rod photoreceptor cells. ARL3 dysregulation, i.e. constitutive activation via ARL3-Q71L transgenic expression, leads to disrupted trafficking of prenylated proteins, including PDE6, GRK1 and transducin. Additionally, we show that active ARL3-Q71L and not ARL3-WT interacts with $\operatorname{PrBP} \delta$ in vivo. 


\section{RESULTS}

\section{ARL3-Q71L Dominant Active Mutant Transgenic Model Generation}

To determine the role for ARL3 in photoreceptor function, we generated a transgenic animal expressing dominant active mouse ARL3-Q71L in rod photoreceptor cells (Fig. 1a). A control transgenic line expressing tagged wild type mouse ARL3 (ARL3-WT) was also generated. Expression of both wild-type and dominant active ARL3 are under the control of a rhodopsin promoter that drives the expression of transgenes as early as post-natal day 4 (PN4) in rod photoreceptor cells of the retina (15). Transgenic founders were crossed with 129/SV-E wildtype mice over at least two generations to ensure consistent transgene inheritance and expression prior to analysis. Transgenic negative littermates of ARL3-Q71L mice were used as control animals for comparison unless stated otherwise.

We generated an affinity purified rabbit antibody against mouse ARL3. This antibody specifically recognized mouse ARL3 expressed in Human Embryonic Kidney (HEK293) cells (compare lanes 1 vs. 2 in Fig. 1b). Immunoblotting using the custom made antibody comparing the tagged ARL3-Q71L band ( 26 kDa) to the endogenous ARL3 band at $23 \mathrm{kDa}$ (Fig. 1b; p < 0.001) revealed 2.5 fold overexpression of mutant Q71L over endogenous ARL3 levels. Additionally, an anti-HA antibody was used to confirm the identity of the tagged ARL3-Q71L (Fig. 1b (green)). ARL3-Q71L expression was also confirmed by indirect immunofluorescence with anti-ARL3 antibody and anti-HA antibody (Fig. 1c). ARL3-Q71L was found throughout rod photoreceptor cells from the synapse to the OS similar to endogenous ARL3 localization in transgenic negative and transgenic ARL-WT samples (Fig. 1c and Supplementary Fig. 1). Because previous study utilizing immunofluorescence microscopy reported ARL3 to be primarily located in the photoreceptor's connecting cilium (16), we investigated the distribution 
of endogenous ARL3, as well as several other photoreceptor proteins, in serial tangential sections of the retina (Fig. 1d). In this technique, protein detection is based on Western blotting $(17,18)$, which makes it exempt from the tissue fixation artifacts, and it unambiguously confirmed that ARL3 is indeed present throughout the entire photoreceptor layer (Fig. 1d, sections 1-9). A significant fraction of ARL3 was present in the OS, where it co-localized with peripherin/RDS (Fig. 1d, sections 1-2). ARL3 enrichment was also observed in the IS domain that harbors mitochondria, which is adjacent to the connecting cilium, as evident from the presence of the mitochondrial marker, COX I (Fig. 1d, section 3). The low spatial resolution of this technique, however, did not allow us to conclude whether ARL3 is particularly enriched in the connecting cilium. Overall, immunofluorescence data and serial section analysis suggest that transgenic ARL3-Q71L and endogenous ARL3 are found throughout the rod photoreceptor layer including the OS.

\section{Progressive loss of rod photoreceptor function in ARL3-Q71L Mice}

To examine the impact of expressing dominant negative ARL3 on the functioning of photoreceptor cells, we performed electroretinography (ERG) starting from PN20. Transgenic negative littermate controls show a robust "a" wave indicative of photoreceptor function and "b" wave demonstrating an appropriate response from downstream bipolar cells. A similar response was observed in animal models expressing ARL3-Q71L at light intensities under both scotopic and photopic conditions (Fig. 2a). This result indicates that the initial development and function of photoreceptor cells appear unaffected by the expression of ARL3-Q71L. However, there is a progressive loss of rod function and by PN70 scotopic a-wave amplitude is almost completely lost with slightly reduced photopic b-wave amplitude (Fig. 2a and b).

\section{Rod photoreceptor cells degenerate in animals expressing ARL3-Q71L}


Loss of photoreceptor function at PN70 could be due to photoreceptor cell death or defects in the phototransduction cascade. To distinguish these possibilities, we investigated the survival of photoreceptor cells by counting the outer nuclei at various ages of retinal development. At PN30, when there was a substantial reduction in rod photoresponse (463 $\pm 9 \mu \mathrm{V}$ : $\mathrm{Tg}(-)$ vs $327 \pm 56 \mu \mathrm{V}$ : $\operatorname{Tg}(+)$; $\mathrm{p}=0.01$ Fig. 2b), we observed no significant loss in photoreceptor cells. However, by PN70, most of the photoreceptor nuclei was lost in transgenic ARL3-Q71L animals compared to controls. The ONL length was measured at superior and inferior regions of the retina and was not significantly different at PN30, but was dramatically reduced in transgenic animals by PN70 $(\operatorname{Tg}(+)=2.7$ layers and $\operatorname{Tg}(-)=10.3$ layers; $\mathrm{p}<0.001$ at all locations, Fig. $2 \mathrm{~d})$.

\section{Normal elaboration of photoreceptor OS in ARL3-Q71L Mice}

The morphology of rod photoreceptor cells, in particular the structure of photoreceptor outer segment, was analyzed by light and electron microscopy at PN20, when the ERG responses appear normal. Gross morphology using toluidine blue staining on semi-thin sections show normal elaboration of photoreceptor OSs (Fig. 3a). The OS length of the rod photoreceptors in transgenic animals expressing ARL3-Q71L was comparable to that of transgenic negative littermate controls (Supplementary Fig. 2a and 2b; OS length: $\operatorname{Tg}(+)=11.54 \pm 0.12 \mu \mathrm{m}$ and $\mathrm{Tg}(-$ ) $=11.66 \pm 0.10 \mu \mathrm{m} ; \mathrm{p}=0.39)$. However, ultrastructural analysis revealed subtle defects including a slight increase in endosome content within the IS (Fig. 3b; white arrowheads) and misoriented rod OS discs (Fig. 3c; white arrows). The Golgi apparatus and endoplasmic reticulum appear dilated in the retina from ARL3-Q71L transgenic animals (Fig. 3c; Golgi apparatus = black arrows and ER = black arrowheads). These data suggest that overall development of photoreceptor cell outer segments appears to be relatively normal, however, 
ultrastructural changes in both rod inner and outer segments are present due to transgenic expression of ARL3-Q71L.

\section{Expression of ARL3-Q71L reduces the levels of prenylated phototransduction proteins}

The decrease in photoreceptor function despite normal morphology at PN30 suggests that expression of ARL3-Q71L might affect the levels of proteins that participate in phototransduction. In addition, previous in vitro studies suggest a relationship between ARL3 and prenylated proteins through its regulation of $\operatorname{PrBP} \delta(10,11)$. Therefore, we investigated the levels of prenylated phototransduction proteins prior to significant degeneration at PN30. Prenylated phosphodiesterase catalytic subunits $\alpha$ and $\beta$ (PDE6 $\alpha \beta)$ were reduced by $\sim 50 \%$ in rod photoreceptor cells expressing ARL3-Q71L compared to controls (Fig. 4a and 4b; $\mathrm{p}<0.001$ ). Similarly, farnesylated G-protein receptor kinase (GRK1) and transducin $\gamma(\mathrm{T} \gamma)$ levels in retina from ARL3-Q71L animals were reduced by $\sim 40 \%$ and $~ 15 \%$, respectively, compared to controls (Fig. 4a and 4b; GRK1 - $p=0.001, T \gamma-p=0.001$ ). It is important to note that GRK1 is expressed in both rods and cones. Since our animal model expresses ARL3-Q71L exclusively in rods, it is likely that the reduction in GRK1 in rods as tested by western blot is an underestimate. Additionally, other non-prenylated OS proteins remained unchanged including GC1, rod Ta, and rhodopsin ( $>$ > 0.05) as well as rod arrestin (data not shown).

Phosphorylation of activated opsin mediated by GRK1 is necessary for the recovery of photoresponses following a flash of light (19-21). In order to determine if there is a functional loss of GRK1, scotopic double flash (recovery) experiments were done that involve darkadapting the animal and subsequently administering two sequential low intensity (-1.6 Log cd $\mathrm{s} / \mathrm{m}^{2}$ ) flashes (the test flash and the probe flash) with various delays between the two flashes. This provides insight into how quickly rod photoreceptors can recover to produce the same 
response as the test flash. As expected from the reduction in GRK1, recovery of scotopic a-wave was reduced in ARL3-Q71L mice at PN20 compared to controls (Fig. 4c). The scotopic a-wave was completely recovered (i.e. probe flash = test flash) after a $1500 \mathrm{~ms}$ in ARL3-Q71L animals compared to controls in which complete recovery occurred at a 1000 ms delay (Fig. 4c). Overall, these data demonstrate that ARL3-Q71L expression results in a specific reduction in prenylated proteins that more severely affects PDE6 and GRK1 than T $\gamma$ but does not affect other nonprenylated OS proteins.

\section{Loss of rod GRK1 and mislocalization of PDE6 in ARL3-Q71L mice}

After verifying that transgenic expression of ARL3-Q71L caused a specific effect on prenylated protein levels, the localization of those proteins was assessed by immunofluorscence microscopy at PN25 prior to significant cell death. Two distinct defects were noted with respect to different prenylated proteins. In agreement with our results from immunoblotting, we found a marked reduction in immunoreactivity for GRK1 in the rod OS in transgenic ARL3-Q71L animals. As expected, expression of GRK1 in cone outer segments was unaffected (Fig. 5a). Interestingly, transgene expression resulted in mislocalization of PDE6 subunits, and to a lesser extent transducin, to the inner segment in large punctate structures (Fig. 5a-e). Similar to our Tg(-) littermate controls, immunoreactivity of PDE6 $\beta$ in $\operatorname{Tg}(+)$ ARL3-WT was exclusively found in the OS (Supplementary Fig. 1). In addition, other non-prenylated OS proteins were properly localized with comparable intensity, including cyclic nucleotide gated channel $\alpha 1$ (CNGA1) (Fig. 5b-e) and peripherin (Fig. 5a) as well as rhodopsin and GC-1 (data not shown). Furthermore, the punctate structures with immunoreactivity for PDE6 and transducin progressively accumulate with age (Fig. 6a-b). This accumulation of PDE6 in the IS corresponds to a decrease in levels of PDE6 in OS. Additionally, the PDE6 found in these structures is likely 
assembled as it was identifiable with the anti-ROS1 antibody, which is known to recognize only the assembled PDE6 complex (PDE6 $\alpha \beta \gamma$ ) (22). This assembled PDE6 $\alpha \beta \gamma$ was colocalized on the same IS structures as transducin (Fig. 6b). Overall, the data suggest that transgenic expression of ARL3-Q71L results in mislocalization and degradation of prenylated OS proteins.

\section{Accumulation of endosomal vesicles in the retina expressing ARL3-Q71L}

To explore the ultrastructural defects, electron microscopy was performed at PN30 prior to significant cell death at which point accumulation of PDE6 in IS was observed. As expected, general retinal morphology was maintained as shown by semi-thin sections stained with toluidine blue (Fig. 7a). Notably, we observed Muller cell processes extending into the IS through the outer limiting membrane (Fig. 7c; Muller cell = MC). Furthermore, endosome-like structures and misoriented or disrupted OS along with membranous whorls within the IS were frequently observed in PN30 transgenic animals (Fig. 7b and c; top 3 rows; IS membrane whorls = asterisk, endosomes $=$ white arrowheads, disrupted OS $=$ white arrow). . The endosome-like structures tended to be between 1 and $2 \mu \mathrm{m}$ in diameter, which is comparable to the size of the punctate PDE6-containing structures identified by confocal IHC microscopy, suggesting that the prenylated proteins may be localized to endosomal compartments (Fig. 7d; PDE6 panel 1 compared to endosome-like structures (panel 2, 3, 4, 6) and IS membrane whorl (panel 5). Additionally, in ARL3-Q71L animals, the frequency of misoriented / disrupted OS membrane discs was higher and there were fewer well-oriented outer segments compared to controls likely resulting from initial stages of photoreceptor cell death (Fig. 7c, Top Row; white arrows). In conclusion, drastic ultrastructural changes were found in ARL3-Q71L mice, including mislocalized prenylated proteins likely to be accumulating in endosome-like structures within the IS. 


\section{PrBPo interacts with ARL3 in vivo}

To provide mechanistic insight into the defects seen in the ARL3-Q71L transgenic animals, we performed co-immunoprecipitation (co-IP) experiments. We utilized the HA tag to pull-down ARL3-Q71L protein in retinal lysates from transgenic animals. Based on in vitro binding data, we expected that constitutively active ARL3-Q71L would trap $\operatorname{PrBP} \delta$, and thus disable it (10, 23). Here, we have for the first time shown that ARL3-Q71L co-immunoprecipitates $\operatorname{PrBP} \delta$ in vivo while transgenic tagged ARL3-WT does not (Fig. 8). Moreover, we show that PDE6 does not interact with ARL3-Q71L or ARL3-WT (Fig. 8). This indicates that when ARL3-Q71L is bound to $\operatorname{PrBP} \delta$ it does not bind PDE6, which is consistent with previous studies suggesting that ARL3 causes release of prenylated cargo like PDE6 (24).

\section{Defective rod photoreceptor cell migration in ARL3-Q71L retina}

The retina is normally laminated such that the ONL contains only rod and cone photoreceptors and the INL consists of downstream neurons (e.g. bipolar cells). Unexpectedly, we observed a defect in rod photoreceptor cell migration in ARL3-Q71L expressing retina. The migration defect was uniformly observed across the retina (Data not shown). More specifically, a fraction of rod photoreceptor cells, indicated by HA (red) and PDE6 (green) staining, were located in the INL/OPL in adult tissues (Fig. 9a-b). DAPI staining further confirms the identity of these cells as chromatin of rod photoreceptor cells are distinctly more dense compared to downstream neurons $(25,26)$. It should be noted that there was varying severity between different founder lines and the severity of the defect corresponded to the level of transgenic protein expression. Overall, these data suggest that ARL3 plays an important role in the migration of rod photoreceptor cells. 


\section{DISCUSSION}

Regulation and rapid movement of proteins from the photoreceptor inner to outer segment is a poorly understood process and is of clinical significance as defects in this protein trafficking lead to blindness. Due to changes in conformation dependent on nucleotide binding, small GTPases are ideally suited for polarized movement of proteins. In this study, we examined the role of ARL3, a small GTPase, in the trafficking of proteins to the rod photoreceptor outer segment (OS). In addition, mutation or depletion of RP2 associated with XLRP in humans likely results in excess active (GTP-bound) ARL3, similar to the animal model described in this study $(7,13)$. Therefore, our animal model is also useful in understanding the pathogenesis underlying the defects in RP2-associated diseases. In the current study, we show that expression of constitutively active ARL3 (ARL3-Q71L) results in progressive loss of function and degeneration of rod photoreceptor cells. The decline in photoreceptor function was accompanied with progressive accumulation of prenylated PDE6 and transducin in IS. Moreover, levels of farnesylated GRK1 were dramatically reduced. Our study establishes the role of ARL3 in trafficking and stability of prenylated proteins in rod photoreceptor cells.

Importantly, the phenotypes associated with ARL3-Q71L were specific to ARL3 function and not a result of overexpression or transgene insertion as the loss of photoreceptor function corresponded to the level of ARL3-Q71L expression between multiple independent founder lines (Supplementary Fig. 3). The number of PDE6 containing vesicles in the IS also corresponded to the level of transgene expression $\left(\operatorname{Tg}(+++)-11.4 \pm 0.9\right.$ vesicles $/ 1000 \mu \mathrm{m}^{2}$ and $\operatorname{Tg}(++)-2.8 \pm$ 0.2 vesicles $/ 1000 \mu \mathrm{m}^{2} ; \mathrm{p}=0.01$; data not shown). In addition, the defects observed in ARL3Q71L animals were specific as these were not observed in transgenic animals expressing ARL3WT (Supplementary Fig. 1) or ARL2-Q70L (unpublished data). 
Prior to degeneration, we observed no changes in levels or localization of major photoreceptor OS proteins except for prenylated proteins in the ARL3-Q71L transgenic animals. More specifically, levels of PDE6 and GRK1 were severely decreased while transducin levels showed modest reduction at one month old. Immunofluorescence data suggested that these prenylated proteins are trafficked normally to the OS initially, but as the animals aged mislocalization to the IS became more severe. These prenylated proteins accumulated in punctate structures in the IS and the number of these structures increased with age. It should be noted that these structures did not colocalize with the Golgi marker, GM130 and therefore are not Golgi dilations (unpublished data). TEM analysis showed that ARL3-Q71L expression induced endosomal-like structures within the photoreceptor IS, and we hypothesize that misrouted OS proteins were packaged within these structures. The size of the fluorescent puncta identified via immunofluorescence was estimated between 1 and $2 \mu \mathrm{m}$ in diameter, which corresponded to the large endosomal structures frequently found in the IS of TEM sections (Fig. 7). It is likely that prenylated proteins accumulate on the membrane of endosomes due to their lipid modification. This assumption is corroborated by immunofluorescence data, which shows large punctate structures with staining on the circumference of the spherical structure with absent staining in the middle. In addition, staining of these endosomes with the ROS-1 antibody, which recognizes the PDE6 $\alpha \beta \gamma$ complex and not individual subunits, suggests that PDE6 is in fact fully assembled. Importantly, assembled PDE6 and rod transducin $\gamma$ were present on the same puncta within the IS and the number of these puncta increased as the animal aged. Altogether, our results suggest that these prenylated proteins translocate to the OS via a trafficking pathway that is dependent upon proper function of ARL3, and over-activation results in buildup of these proteins in the same subcellular compartment that ultimately leads to their degradation. 
How does ARL3 over-activation result in specific trafficking defects in prenylated proteins? We show that active ARL3-Q71L interacts with PrBPS while ARL3-WT does not. Although the PrBP $\delta$ homolog, UNC119, has been identified as an interacting partner of ARL3 in vitro $(9,23)$, we do not see this interaction in our system. The reason behind this observation is not clear. It is possible that the affinity for UNC119 for ARL3-Q71L is lower and therefore below the detection sensitivity in our experimental system. Our results suggest that mistrafficking of prenylated proteins is due to the sequestration of $\operatorname{PrBP} \delta$ as well as its homologs by the dominant active ARL3-Q71L. For PrBP $\delta$, this would result in a predominant "closed" conformation and loss of the ability to bind prenylated proteins. The mislocalization of prenylated proteins in ARL3-Q71L expressing mice is also in agreement with findings from knockout of $\operatorname{PrBP} \delta$ or RP2, the GAP for ARL3 $(7,12,14)$.

Photoreceptor degeneration in this ARL3-Q71L mouse model is rapid and nearly complete by PN70. The primary cause of the rapid photoreceptor loss is the ability of ARL3 to sequester multiple binding partners including PrBP $\delta$ due to defective GTP hydrolysis by ARL3-Q71L. In addition to its potential role in prenylated protein trafficking, ARL3 is also thought to participate in regulation of dynein motors, Golgi maintenance and vesicle dispersion, and microtubule stability (16, 27-32). However, we observed normal trafficking of non-prenylated proteins suggesting that dynein motors and microtubules are not affected. A moderate Golgi and ER dilation was noted specifically in transgenic animals. We believe this is due to the excessive accumulation of prenylated proteins in the endomembrane system as they cannot be properly retrieved by $\operatorname{PrBP} \delta$ or its homologs. Further studies are needed to test this hypothesis.

Surprisingly, we observed defective migration of a fraction of ARL3-Q71L expressing photoreceptor cells. These cells remained in the INL and in the OPL instead of migrating to the 
ONL. It is likely only a fraction of photoreceptor cells express ARL3-Q71L prior to migration since expression under the $4.4 \mathrm{~kb}$ rhodopsin promoter is detectable at PN4 (15) while the majority of photoreceptor nuclei have migrated to the outer layer by PN6 (33). Interestingly, defective migration of nuclei is also present in mice lacking RP2 where ARL3 is thought to be overactive (13; refer to Fig. 4). We hypothesize that this migration defect results from instability or improper localization of the farnesylated lamins. Depletion of lamins results in neurodevelopmental phenotypes, including a severe defect in neuronal migration and nuclear abnormalities such as blebs and elongation $(34,35)$. Similarly, we observed blebs in the nuclear envelope (Supplementary Fig. 4). Additionally, it was recently found that $\operatorname{PrBP} \delta$ interacts with lamin B (36). Alternatively, ARL3 has been shown to affect cytokinesis through a microtubule function-based mechanism which could ultimately affect proper lamination of photoreceptor cells (30). Importantly, ARL3 is ubiquitously expressed throughout different tissues including the brain (37), suggesting that it could be involved in the process of neuronal migration in the CNS. Disruption of this process results in lethal and non-lethal neurodevelopment defects including lissencephaly, epilepsy, schizophrenia and autism (38-41). In conclusion, this novel ARL3 function has broad implications in neuronal migration and associated disease, and therefore requires further research.

Based on the results obtained in this study, we have developed a mechanistic model of ARL3 function in rod photoreceptor cells and how this mechanism goes awry in the presence of a dominant active ARL3 mutant (Fig. 10). After addition of a prenyl group by a specific prenyl transferase, prenylated proteins such as PDE6, GRK1 and transducin are targeted to ER as the last two steps of prenyl processing, endoproteolysis and methylation, are mediated by ERmembrane bound enzymes. How these prenylated proteins are then moved to the OS from the 
ER compartment still remains a mystery. Based on our results and previous studies, we believe that $\operatorname{PrBP} \delta$ and its functional homologs can retrieve the prenylated proteins from the ER membrane for further transport to the destination membrane similar to the proposed transport of K-ras from the ER to the plasma membrane $(42,43)$. This process must be regulated by ARL3 such that the proteins are selectively released at the appropriate location. In order for this to occur, the recently identified GEF of ARL3, ARL13b (44), must be localized in the area of the destination membrane such that ARL3 can be activated and bind $\operatorname{PrBP} \delta$ or its homologue causing release of the cargo protein, i.e. PDE6, GRK1, or transducin. Interestingly, recent research shows that ARL13b localizes to the cilium and OS region (45), which is corroborated by our results (Supplementary Fig. 5). This finding suggests that ARL3-mediated release of cargo occurs at the cilium or in the OS. Additionally, the ARL3-PrBP $\delta$ complex can then be acted on by RP2, which facilitates hydrolysis of GTP to GDP thereby inactivating ARL3 and causing dissociation and recycling of $\operatorname{PrBP} \delta$ and its homologues. In our dominant active transgenic ARL3-Q71L model, it is likely that this complex remains intact and sequesters $\operatorname{PrBP} \delta$ from its transport cycle of prenylated proteins (Fig. 10b). Disruption of the transport of prenylated proteins results in their accumulation in IS and sorting to a degradation pathway.

Our study sheds light on the process of targeted protein transport and our current model shows the importance of ARL3 regulation in trafficking of prenylated phototransduction proteins. Finally, we believe our animal model mimics human XLRP resulting from RP2 mutations that disrupt the small GTPase cycle of ARL3. 


\section{MATERIALS AND METHODS}

\section{Generation of ARL3-Q71L transgenic mice}

Full-length mouse Arl3 was amplified from a clone purchased from a commercial vendor (Thermo Scientific). Using polymerase chain reactions (PCR), we engineered a tandem Cterminal hemagglutinin (HA) and FLAG fusion tags and subsequently sub-cloned this PCR product behind a $4.4 \mathrm{~kb}$ rhodopsin promoter (Rhop) (15).

ARL3-Q71L dominant active mutant was constructed with the full-length tandem tagged Arl3 as the template. A mutant oligonucleotide with specific change altering glutamine (Q) at position 71 to leucine (L) (WV832: 5’-TCT GAT TTT CCT CAG CCC GCC AAT GTC-3') was used to create the Arl3 mutant with the use of megaprimer directed site-directed mutagenesis method. The megaprimer was further extended and amplified using flanking primers containing SalI and BamHI restriction sites. The PCR product was then cloned behind $4.4 \mathrm{~kb}$ rhodopsin promoter using SalI and BamHI restriction sites. All clones selected were sequenced in both directions to confirm that there are no unintended mutations.

The plasmid backbone from clones containing Rhop-Arl3 (wild-type) and Rhop-Arl3 Q71L (mutant) was removed by digestion with KpnII and NotI enzymes and purified using agarose gel electrophoresis followed by elution of the fragment containing Rhop-Arl3 (or Arl3Q71L)-polyA. The purified DNA fragment was injected into the pronuclei of oocytes from superovulated FVB/N females (WVU Transgenic Animal Core Facilities) and implanted into pseudo-pregnant CD-1 females.

\section{Genotyping and Founder Line Maintenance}


Wild-type ARL3 (ARL3-WT) and mutant ARL3-Q71L transgenic founders were identified by PCR using either genomic DNA prepared from tail or ear punches: (5' GGA TCG TGA ATC AGC CTC TGG CTT 3’; and 5’ TTT CTT CTT TGC GTT GAC ATT CTT GC 3’). All PCR reactions were conducted using NEB quick load Taq polymerase with following conditions, $95^{0} \mathrm{C}-2$ minutes, $95^{0} \mathrm{C}-30$ seconds, $59^{0} \mathrm{C}-30$ seconds, $72^{0} \mathrm{C}-45$ seconds with last three steps repeated for 33 cycles followed by a final extension step of $72^{\circ} \mathrm{C}$ at 5 minutes. Founders were backcrossed into wild-type 129/SV-E mice (Charles River) to eliminate the Pde6b ${ }^{r d 1}$ mutation present in the FVB founders. Removal of $r d 1$ allele was confirmed by PCR genotyping (46). The animals were maintained under 12-h light / 12-h dark light cycles with food and water provided ad libitum. All experimental procedures involving animals in this study were approved by Institutional Animal Care and Use Committee of the West Virginia University.

\section{Electroretinographic (ERG) analysis}

Mice were dark-adapted overnight prior to testing. Test subject eyes were topically dilated with a 1:1 mixture of tropicamide:phenylephrine hydrochloride. For ERG testing, mice were placed on a heated platform with continuous flow of isoflurane anesthesia through a nose cone $(1.5 \%$ isoflurane with 2.5 liters per minute (lpm) oxygen flow rate). A reference electrode was placed subcutaneously in the scalp. ERG responses were recorded from both eyes with silver wire electrodes placed on each cornea, with contact being made with a drop of hypromellose solution (2\% hypromellose in PBS) (Gonioscopic Prism Solution, Wilson Ophthalmic, Mustang, OK, USA). Rod dominated responses were elicited in the dark with flashes of LED white light at increasing flash intensities. Light-adapted cone responses were elicited with white light flashes in the presence of a $30 \mathrm{~cd} / \mathrm{m}^{2}$ rod-saturating white background light. ERGs were performed on the UTAS Visual Diagnostic System with BigShot Ganzfeld with UBA-4200 amplifier and 
interface, and EMWIN 9.0.0 software (LKC Technologies, Gaithersburg, MD, USA). Double flash ERG was performed just as above except to flashes were administered at -1.6 LogI for scotopic (rod) recovery and 2.4 LogI (under background light as above) for photopic (cone) recovery. The recovery (probe) flashes were elicited at variable delays after the initial (test flash) (500, 750, 1000, or $1500 \mathrm{~ms}$ after the test flash).

\section{Generation of Antibody against full-length ARL3}

ARL3 protein was purified from pTriex-4 vector expressing a C-terminal his-tagged ARL3 protein in Origami E.coli strain (Novagen). Origami cells were grown to an $\mathrm{OD}_{600} \approx 0.6$ and protein production was induced with $1 \mathrm{mM}$ IPTG for 18 hours at $18^{\circ} \mathrm{C}$. Finally, the protein was purified from IPTG induced E.coli soluble fraction using a Nickel His Affinity Column and purified protein was supplied to Pacific Immunology Corp. for generation of the antibody in rabbits. The antibody serum was affinity-purified using a GST-ARL3 fusion protein. Purified antibody was then tested by western blot and immunofluorescence in HEK 293 cells expressing recombinant ARL3 and retinal extracts from animals expressing ARL3.

\section{Immunoblots}

Mice were euthanized by $\mathrm{CO}_{2}$ inhalation and eyes were enucleated. For immunoblots, flash frozen retinal samples were dissected from enucleated eyes and sonicated in phosphate buffered

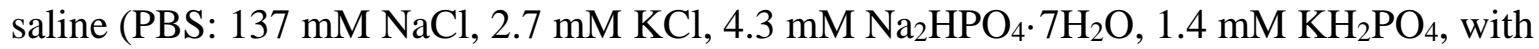
protease inhibitor cocktail (Roche)). Protein concentrations were measured using a NanoDrop spectrophotometer (Thermo Fisher Scientific, Inc). Samples were loaded at $150 \mu$ g total protein per well of a 4-20\% gradient polyacrylamide SDS-PAGE resolving gel (Criterion Midi format, Bio-Rad), resolved and transferred onto polyvinylidene difluoride membranes (Immunobilon-FL, 
Millipore, Billerica). Membranes were blocked with western blot blocking buffer (Rockland Inc.) for $30 \mathrm{~min}$ at room temperature and incubated with the indicated primary antibodies below overnight at $4^{\circ} \mathrm{C}$. Blots were washed in PBST (PBS with 0.1\% Tween-20) 3 times for 5 minutes each wash (3 X 5 min) at room temperature and incubated in secondary antibody, goat anti-rabbit Alexa 680 (or 800), goat anti-rat Alexa 800, or goat anti-mouse Alexa 680 (Invitrogen) for 30 min at room temperature. After 3 x 5 minutes of washes with PBST, membranes were scanned using the Odyssey Infrared Imaging System (LI-COR Biosciences, Lincoln, NE, USA). The following primary antibodies were used in this study at a dilution of 1:2000 unless otherwise noted: rat anti-HA antibody (Roche), rabbit anti-PDE6 $\alpha$ (Pierce), rabbit anti-PDE6 $\beta$ (Pierce), rabbit anti-PDE6 $\gamma$ (Pierce), assembled PDE6 (ROS1) (generously provided by Drs. Ted Wensel (Baylor College) and Rick Cote (University of New Hampshire), rabbit anti-Transducin- $\alpha$ t1 (Santa Cruz), mouse anti-cytochrome c oxidase subunit I (COX I) (MS404 MitoSciences), rabbit anti-Transducin- $\gamma t 1$ (Santa Cruz), mouse anti-GRK1 (Thermo Fisher), mouse anti-CNGA1 (UC Davis/NIH NeuroMab Facility), rabbit anti-RDS-c (Peripherin) (Gabriel Travis, University of California, Los Angeles, CA), mouse anti-1D4 (rhodopsin) (gift from Dr. Ted Wensel, Baylor Collect of Medicine), and rabbit anti-ARL3 (1:1000) (this work). DAPI (1:1000) (4',6diamindino-2-phenylindole, Invitrogen) and propidium iodide (PI) (1:2000) (EMD Millipore, Billerica, MA, USA) were also used in indirect immunofluorescence.

\section{Immunoprecipitation (IP)}

Frozen retinas (n=4) from ARL3-Q71L, ARL3-WT, or transgenic negative animals were homogenized in $400 \mu \mathrm{l}$ of $1 \mathrm{X}$ PBS containing protease inhibitors and $1 \mathrm{mM}$ iodoacteamide using sonication (Fisher Scientific). The homogenized retinal extracts were centrifuged at 10,000 X g for 5 minutes and supernatant from this step was used for IP. The supernatant was then incubated 
for 2 hours with washed anti-HA beads (Roche Biochemicals) at $4^{\circ} \mathrm{C}$, followed by three rounds of washes with PBS $+0.1 \%$ TX-100, one wash with PBS, and final elution with HA peptide at $37^{\circ} \mathrm{C}$ for 1 hour. Proteins were then separated by 4-20\% gradient SDS-PAGE and immunoblot was performed as described above.

\section{Immunolocalization}

For indirect immunofluorescence studies, enucleated eyes were immersed in 4\% paraformaldehyde fixative for 5 minutes prior to removal of the anterior segments. Eyecups were fixed for an additional 1 hour, washed in PBS for 20 minutes, incubated in 20\% sucrose in PBS overnight at $4^{\circ} \mathrm{C}$. Eyes were then incubated in 1:1 mixture of 20\% sucrose in PBS:OCT (Cryo Optimal Cutting Temperature Compound, Sakura) for 1 hour, and flash frozen in OCT. Cryosectioning was performed with a Leica CM1850 Cryostat, and serial retinal sections of 16 $\mu \mathrm{m}$ thickness were mounted on Superfrost Plus slides (Fisher Scientific). Retinal sections mounted on slides were washed with PBS, and incubated for 1 hour in blocking buffer (PBS with 5\% goat sera, $0.5 \%$ TritonX-100, $0.05 \%$ sodium azide). Retinal sections were incubated with primary antibody at the dilutions indicated below for overnight at $4^{\circ} \mathrm{C}$, followed by two 15 minutes washes with PBS $+0.1 \%$ TX-100, and one 10 minutes wash with PBS before incubation with secondary antibody at a 1:1000 dilution (DAPI nuclear stain 405, anti-Rat 568, anti-Rabbit 488 (or 568), anti-mouse 488 (or 568)) for 1 hour. Slides were mounted with ProLong Gold (Life

Technologies) and cover slipped. Confocal imaging was performed at the WVU Microscope Imaging Facility with a Zeiss LSM 510 laser scanning confocal on a LSM Axioimager upright microscope using excitation wavelengths of 405, 488, and $543 \mathrm{~nm}$.

\section{Hematoxylin \& Eosin Immunohistochemical Analysis}


Enucleated eyes were marked at the 12 o'clock position for reference with a red lipophilic dye and fixed in Excalibur Pathology Fixation Buffer at room temperature. These fixed whole eyes were then sent to Excalibur Pathology (Norman, OK) for paraffin embedding, sectioning at $2 \mu \mathrm{m}$ and H\&E staining. The stained sections were then imaged on a Zeiss light microscope. Analysis included measuring OS length from 3 different animals at 3 regions ventral and 3 regions dorsal to the optic nerve.

\section{Ultrastructural Analysis}

Enucleated eyes were fixed (2\% paraformaldehyde, 2.5\% glutaraldehyde, $0.1 \mathrm{M}$ cacodylate buffer, $\mathrm{pH}$ 7.5) for 30 minutes prior to dissection and removal of the anterior segment and lens, and extensively fixed for 48 hours at room temperature. The fixed eyecup was dissected into 6-8 wedge-shaped pieces. Wedges were dehydrated in a graded ethanol series, and then embedded in Polybed 812 (PolySciences, Inc., Warrington, PA, USA). Semi-thin $(1 \mu \mathrm{m})$ sections were collected onto glass slides, stained with toluidine blue, and visualized using a Zeiss Axioimager 2 microscope equipped with EC Plan-Neofluar 40x (N.A. 0.75) and 100x (1.3 N.A.) objectives. Thin sections (ca. $80 \mathrm{~nm}$ ) from selected wedges were collected onto nickel grids, stained with 2\% uranyl acetate and lead citrate, and imaged using an FEI Morgagni transmission electron microscope at $80 \mathrm{kV}$. 


\section{ACKNOWLEDGEMENTS}

We thank Dr. Peter Mathers and Ms. Ingrid Weterrings of the West Virginia University (WVU) Transgenic Animal Core Facility for their help and services in generating our transgenic lines and Dr. Karen Martin of the WVU Microscope Imaging Facility. We thank Drs. Rick Cote, Ted Wensel, and Gabriel Travis for sharing antibodies generated in their laboratories. We are indebted to the members of the Ramamurthy laboratory for their help and support throughout this study.

\section{FUNDING}

This work was supported by grants from the National Institutes of Health (EY017035 (to V.R.), EY019665 (to M.S.), R01EY013246 (to A.F.X.G.), RR017890 (to A.F.X.G.), West Virginia Lions, Lions Club International Fund and an Unrestricted Challenge Grant from Research to Prevent Blindness (RPB). 
CONFLICTS OF INTEREST

None 


\section{REFERENCES}

1. Sung,C.H. and Chuang,J.Z. (2010) The cell biology of vision. J. Cell Biol., 190, 953-963.

2. Pearring,J.N., Salinas,R.Y., Baker,S. a. and Arshavsky,V.Y. (2013) Protein sorting, targeting and trafficking in photoreceptor cells. Prog. Retin. Eye Res., 36, 24-51.

3. Schrick,J.J., Vogel,P., Abuin,A., Hampton,B. and Rice,D.S. (2006) ADP-ribosylation factorlike 3 is involved in kidney and photoreceptor development. Am. J. Pathol., 168, 12881298.

4. Gillingham,A.K. and Munro,S. (2007) The small G proteins of the Arf family and their regulators. Annu. Rev. Cell Dev. Biol., 23, 579-611.

5. Veltel,S., Gasper,R., Eisenacher,E. and Wittinghofer,A. (2008) The retinitis pigmentosa 2 gene product is a GTPase-activating protein for Arf-like 3. Nat. Struct. Mol. Biol., 15, 373380.

6. Branham,K., Othman,M., Brumm,M., Karoukis,A.J., Atmaca-Sonmez,P., Yashar,B.M., Schwartz,S.B., Stover,N.B., Trzupek,K., Wheaton,D., et al. (2012) Mutations in RPGR and RP2 account for $15 \%$ of males with simplex retinal degenerative disease. Investig. Ophthalmol. Vis. Sci., 53, 8232-8237.

7. Zhang,H., Hanke-Gogokhia,C., Jiang,L., Li,X., Wang,P., Gerstner,C.D., Frederick,J.M., Yang,Z. and Baehr,W. (2015) Mistrafficking of prenylated proteins causes retinitis pigmentosa 2. FASEB J., 29, 932-942.

8. Bartolini,F., Bhamidipati,A., Thomas,S., Schwahn,U., Lewis,S. a. and Cowan,N.J. (2002) Functional overlap between retinitis pigmentosa 2 protein and the tubulin-specific chaperone cofactor C. J. Biol. Chem., 277, 14629-14634.

9. Ismail,S. a, Chen,Y.-X., Miertzschke,M., Vetter,I.R., Koerner,C. and Wittinghofer,A. (2012) Structural basis for Arl3-specific release of myristoylated ciliary cargo from UNC119. EMBO J., 31, 4085-4094.

10. Wätzlich,D., Vetter,I., Gotthardt,K., Miertzschke,M., Chen,Y.-X., Wittinghofer,A. and Ismail,S. (2013) The interplay between RPGR, PDE $\delta$ and Arl2/3 regulate the ciliary targeting of farnesylated cargo. EMBO Rep., 14, 465-72.

11. Ismail,S. a, Chen,Y.-X., Rusinova,A., Chandra,A., Bierbaum,M., Gremer,L., Triola,G., Waldmann,H., Bastiaens,P.I.H. and Wittinghofer,A. (2011) Arl2-GTP and Arl3-GTP regulate a GDI-like transport system for farnesylated cargo. Nat. Chem. Biol., 7, 942-949.

12. Zhang,H., Li,S., Doan,T., Rieke,F., Detwiler,P.B., Frederick,J.M. and Baehr,W. (2007) Deletion of PrBP/delta impedes transport of GRK1 and PDE6 catalytic subunits to photoreceptor outer segments. Proc. Natl. Acad. Sci. U. S. A., 104, 8857-8862.

13. Li,L., Khan,N., Hurd,T., Ghosh,A.K., Cheng,C., Molday,R., Heckenlively,J.R., Swaroop,A. and Khanna,H. (2013) Ablation of the X-linked retinitis pigmentosa 2 (Rp2) gene in mice results in opsin mislocalization and photoreceptor degeneration. Investig. Ophthalmol. Vis. Sci., 54, 4503-4511. 
14. Liu,F., Chen,J., Yu,S., Raghupathy,R.K., Liu,X., Qin,Y., Li,C., Huang,M., Liao,S., Wang,J., et al. (2015) Knockout of RP2 decreases GRK1 and rod transducin subunits and leads to photoreceptor degeneration in zebrafish. Hum. Mol. Genet., 10.1093/hmg/ddv197.

15. Song,H., Bush,R. a, Vijayasarathy,C., Fariss,R.N., Kjellstrom,S. and Sieving,P. a (2014) Transgenic expression of constitutively active RAC1 disrupts mouse rod morphogenesis. Invest. Ophthalmol. Vis. Sci., 55, 2659-68.

16. Grayson,C., Bartolini,F., Chapple,J.P., Willison,K.R., Bhamidipati,A., Lewis,S. a, Luthert,P.J., Hardcastle,A.J., Cowan,N.J. and Cheetham,M.E. (2002) Localization in the human retina of the X-linked retinitis pigmentosa protein RP2, its homologue cofactor $\mathrm{C}$ and the RP2 interacting protein Arl3. Hum. Mol. Genet., 11, 3065-3074.

17. Song,H. and Sokolov,M. (2009) Analysis of protein expression and compartmentalization in retinal neurons using serial tangential sectioning of the retina. J. Proteome Res., 8, 346-351.

18. Belcastro,M., Song,H., Sinha,S., Song,C., Mathers,P.H. and Sokolov,M. (2012) Phosphorylation of phosducin accelerates rod recovery from transducin translocation. Invest. Ophthalmol. Vis. Sci., 53, 3084-91.

19. Lyubarsky, a L., Chen,C., Simon,M.I. and Pugh,E.N. (2000) Mice lacking G-protein receptor kinase 1 have profoundly slowed recovery of cone-driven retinal responses. J. Neurosci., 20, 2209-2217.

20. Cideciyan, a V, Zhao,X., Nielsen,L., Khani,S.C., Jacobson,S.G. and Palczewski,K. (1998) Null mutation in the rhodopsin kinase gene slows recovery kinetics of rod and cone phototransduction in man. Proc. Natl. Acad. Sci. U. S. A., 95, 328-33.

21. Chen,C.K., Burns,M.E., Spencer,M., Niemi,G. a, Chen,J., Hurley,J.B., Baylor,D. a and Simon,M.I. (1999) Abnormal photoresponses and light-induced apoptosis in rods lacking rhodopsin kinase. Proc. Natl. Acad. Sci. U. S. A., 96, 3718-3722.

22. Kolandaivelu,S., Singh,R.K. and Ramamurthy,V . (2014) AIPL1, a protein linked to blindness, is essential for the stability of enzymes mediating cGMP metabolism in cone photoreceptor cells. Hum. Mol. Genet., 23, 1002-1012.

23. Wright,K.J., Baye,L.M., Olivier-Mason,A., Mukhopadhyay,S., Sang,L., Kwong,M., Wang,W., Pretorius,P.R., Sheffield,V.C., Sengupta,P., et al. (2011) An ARL3-UNC119RP2 GTPase cycle targets myristoylated NPHP3 to the primary cilium. Genes Dev., 25, 2347-2360.

24. Baehr,W. (2014) Membrane Protein Transport in Photoreceptors: The Function of PDE . Invest. Ophthalmol. Vis. Sci., 55, 8653-8666.

25. Kizilyaprak,C., Spehner,D., Devys,D. and Schultz,P. (2010) In vivo chromatin organization of mouse rod photoreceptors correlates with histone modifications. PLoS One, 5, e11039.

26. Carter-Dawson,L.D. and LaVail,M.M. (1979) Rods and cones in the mouse retina. I. Structural analysis using light and electron microscopy. J. Comp. Neurol., 188, 245-62.

27. Jin,M., Yamada,M., Arai,Y., Nagai,T. and Hirotsune,S. (2014) Arl3 and LC8 regulate dissociation of dynactin from dynein. Nat. Commun., 5, 5295. 
28. Kim,H., Xu,H., Yao,Q., Li,W., Huang,Q., Outeda,P., Cebotaru,V., Chiaravalli,M., Boletta,A., Piontek,K., et al. (2014) Ciliary membrane proteins traffic through the Golgi via a Rabep1/GGA1/Arl3-dependent mechanism. Nat. Commun., 5, 5482.

29. Kahn,R. a, Volpicelli-Daley,L., Bowzard,B., Shrivastava-Ranjan,P., Li,Y., Zhou,C. and Cunningham,L. (2005) Arf family GTPases: roles in membrane traffic and microtubule dynamics. Biochem. Soc. Trans., 33, 1269-1272.

30. Zhou,C., Cunningham,L., Marcus,A., Li,Y. and Kahn,R.A. (2006) Arl2 and Arl3 Regulate Different Microtubule-dependent Processes. Mol. Biol. Cell, 17, 2476-2487.

31. Zhang,Q., Hu,J. and Ling,K. (2013) Molecular views of Arf-like small GTPases in cilia and ciliopathies. Exp. Cell Res., 319, 2316-2322.

32. Evans,R.J., Schwarz,N., Nagel-Wolfrum,K., Wolfrum,U., Hardcastle,A.J. and Cheetham,M.E. (2010) The retinitis pigmentosa protein RP2 links pericentriolar vesicle transport between the Golgi and the primary cilium. Hum. Mol. Genet., 19, 1358-1367.

33. Yu,J., Lei,K., Zhou,M., Craft,C.M., Xu,G., Xu,T., Zhuang,Y., Xu,R. and Han,M. (2011) KASH protein Syne-2/Nesprin-2 and SUN proteins SUN1/2 mediate nuclear migration during mammalian retinal development. Hum. Mol. Genet., 20, 1061-1073.

34. Adam,S. a, Butin-Israeli,V., Cleland,M.M., Shimi,T. and Goldman,R.D. (2013) Disruption of lamin B1 and lamin B2 processing and localization by farnesyltransferase inhibitors. Nucleus, 4, 142-50.

35. Coffinier,C., Jung,H.-J., Nobumori,C., Chang,S., Tu,Y., Barnes,R.H., Yoshinaga,Y., de Jong,P.J., Vergnes,L., Reue,K., et al. (2011) Deficiencies in lamin B1 and lamin B2 cause neurodevelopmental defects and distinct nuclear shape abnormalities in neurons. Mol. Biol. Cell, 22, 4683-93.

36. Humbert,M.C., Weihbrecht,K., Searby,C.C., Li,Y., Pope,R.M., Sheffield,V.C. and Seo,S. (2012) ARL13B, PDE6D, and CEP164 form a functional network for INPP5E ciliary targeting. Proc. Natl. Acad. Sci. U. S. A., 109, 19691-6.

37. Cavenagh,M., Breiner,M., Schürmann,A., Rosenwald,A., Terui,T., Zhang,C., Randazzo,P., Adams,M., Joost,H. and Kahn,R. (1994) ADP-ribosylation factor (ARF)-like 3, a new member of the ARF family of GTP-binding proteins cloned from human and rat tissues. $J$. Biol. Chem., 269, 18937-42.

38. Wynshaw-Boris, a. and Gambello,M.J. (2001) LIS1 and dynein motor function in neuronal migration and development. Genes Dev., 15, 639-651.

39. Kähler,A.K., Djurovic,S., Kulle,B., Jönsson,E.G., Agartz,I., Hall,H., Opjordsmoen,S., Jakobsen,K.D., Hansen,T., Melle,I., et al. (2008) Association analysis of schizophrenia on 18 genes involved in neuronal migration: MDGA1 as a new susceptibility gene. Am. J. Med. Genet. B. Neuropsychiatr. Genet., 147B, 1089-100.

40. Deutsch,S.I., Burket,J.A. and Katz,E. (2010) Does subtle disturbance of neuronal migration contribute to schizophrenia and other neurodevelopmental disorders? Potential genetic mechanisms with possible treatment implications. Eur. Neuropsychopharmacol., 20, 281-7.

41. Wegiel,J., Kuchna,I., Nowicki,K., Imaki,H., Wegiel,J., Marchi,E., Ma,S.Y., Chauhan,A., 
Chauhan,V., Bobrowicz,T.W., et al. (2010) The neuropathology of autism: Defects of neurogenesis and neuronal migration, and dysplastic changes. Acta Neuropathol., 119, 755770 .

42. Chandra,A., Grecco,H.E., Pisupati,V., Perera,D., Cassidy,L., Skoulidis,F., Ismail,S. a., Hedberg,C., Hanzal-Bayer,M., Venkitaraman,A.R., et al. (2012) The GDI-like solubilizing factor PDE $\delta$ sustains the spatial organization and signalling of Ras family proteins. Nat. Cell Biol., 14, 329-329.

43. Apolloni, a, Prior,I. a, Lindsay,M., Parton,R.G. and Hancock,J.F. (2000) H-ras but not K-ras traffics to the plasma membrane through the exocytic pathway. Mol. Cell. Biol., 20, 24752487.

44. Gotthardt,K., Lokaj,M., Koerner,C., Falk,N., Gießl,A. and Wittinghofer,A. (2015) A Gprotein activation cascade from Arl13B to Arl3 and implications for ciliary targeting of lipidated proteins. Elife.

45. Datta,P., Allamargot,C., Hudson,J.S., Andersen,E.K., Bhattarai,S., Drack,A. V., Sheffield,V.C. and Seo,S. (2015) Accumulation of non-outer segment proteins in the outer segment underlies photoreceptor degeneration in Bardet-Biedl syndrome. Proc. Natl. Acad. Sci., 112, E4400-E4409.

46. Giménez,E. and Montoliu,L. (2001) A simple polymerase chain reaction assay for genotyping the retinal degeneration mutation (Pdeb(rd1)) in FVB/N-derived transgenic mice. Lab. Anim., 35, 153-156. 


\section{FIGURE LEGENDS}

Figure 1. ARL3-Q71L Dominant Active Mutant Transgenic Model Generation. A. Scheme showing the construct used to express ARL3 in rod photoreceptors cells. The Arl3-Q71L (glutamine to leucine) dominant active mutant was expressed under a $4.4 \mathrm{~Kb}$ rhodopsin promoter. This mutant protein was also C-terminally tagged with FLAG and Hemagglutinin (HA). B. Western blot analysis of HEK 293 cells transfected with empty vector or wild-type ARL3 (lanes 1 and 2) and retinal lysate from Tg(-) and Tg(+) ARL3-Q71L animals (lanes 3 and 4). Staining was done with antibodies directed at ARL3 (red) and HA (green) to compare expression levels of transgenic vs. endogenous ARL3 (refer to bar graph; p < 0.001). C. Retinal sections from $\operatorname{Tg}(-)$ and $\operatorname{Tg}(+)$ animals stained for ARL3 (green) and CNGA1 (red) (Top and Middle Panel) and ARL3 (green) and HA (red) (Bottom Panel) (Scale bar $=10 \mu \mathrm{m})$. D. Representative immunoblots showing the distribution of ARL3 and other proteins in a set of serial tangential $10 \mu \mathrm{m}$ sections (1-10) of the mouse retina. RDS (perpherin) was used as a rod OS marker, COX I was used as a mitochondrial marker present in rod IS and synapse, the $\alpha$ subunit of rod transducin $(\operatorname{rod} \mathrm{T} \alpha)$ was used as a general rod marker, and $\beta$-tubulin was used as a general cellular marker. The inset on top, showing a mouse retinal section stained with toluidine blue, is provided to illustrate the origin of each section from various cellular compartments of rod photoreceptors.

\section{Figure 2. Progressive loss of rod photoreceptor function and degeneration in ARL3-Q71L} retina. A. Representative scotopic (rod) and photopic (cone) electroretinograms (ERGs) comparing Tg(-) and Tg(+) animals at PN20 and PN70 across multiple light intensities. B. Graph illustrating the scotopic a-wave amplitude measured at the light intensity of $0 \mathrm{Log} C \mathrm{~d}^{*} \mathrm{~s} / \mathrm{m}^{2}$ as a function of age. C. Retinal sections showing nuclei from $\operatorname{Tg}(-)$ and $\operatorname{Tg}(+)$ animals stained with 
propidium iodide at PN30 and PN70. D. Quantification of the ONL length (number of cell layers) at different locations within the retina from the inferior to superior portion between $\mathrm{Tg}(-)$ and $\operatorname{Tg}(+)$ animals at PN30 and PN70. $*=\mathrm{p}<0.01$.

Figure 3. Normal elaboration of photoreceptor OS in ARL3-Q71L Mice. A. Semi-thin sections of $\operatorname{Tg}(-)$ and $\operatorname{Tg}(+)$ retina from PN20 littermates stained with toluidine blue (scale bar = $20 \mu \mathrm{m})$. B. Low magnification images from the OS and IS with endosomes indicated by white arrowheads (scale bar $=2 \mu \mathrm{m}$ ). C. High magnification ultrastructure of the OS. White arrows point to membrane whorls in the rod OS from $\mathrm{Tg}(+)$ retina. Dilated endomembranes in the rod IS including Golgi apparatus indicated by black arrows and rough endoplasmic reticulum indicated by black arrowheads (scale bar $=0.5 \mu \mathrm{m}$ ).

Figure 4. Expression of ARL3-Q71L reduces the levels of prenylated phototransduction proteins. A. Representative immunoblots of PN30 retinal protein samples showing levels of prenylated OS proteins and non-prenylated controls. B. Quantification of protein levels from $\operatorname{Tg}(-)$ and $\operatorname{Tg}(+)$ retinal isolates $($ PDE6 $\beta: \mathrm{p}=0.0004$, PDE6 $\alpha: \mathrm{p}=0.009$, GRK1 $: \mathrm{p}=0.001, \mathrm{~T} \gamma$ : $\mathrm{p}=0.001)$. C. Scotopic double flash (recovery) at PN20 shows the recovery of a-wave amplitude at different latencies between flashes from $\mathrm{Tg}(-)$ and $\operatorname{Tg}(+)$ animals $(500 \mathrm{~ms}-\mathrm{p}<0.002$; $750 \mathrm{~ms}$ $-\mathrm{p}<0.001 ; 1000 \mathrm{~ms}-\mathrm{p}<0.002 ; 1500 \mathrm{~ms}-\mathrm{p}=0.32) .{ }^{*}$ indicates statistically significant difference.

Figure 5. Loss of rod GRK1 and mislocalization of PDE6 in ARL3-Q71L mice. Immunolocalization of OS proteins in retinal cross sections at PN25. A. GRK1 (green) counterstained with peripherin/RDS (red) in $\mathrm{Tg}(-)$ and $\mathrm{Tg}(+)$ sections. B. PDE6 $\beta$ (green) and C. PDE6 $\gamma$ (green) each counterstained with CNGA1 (red) in $\operatorname{Tg}(-)$ and $\operatorname{Tg}(+)$ sections. Mislocalization of PDE6 $\beta$ or PDE6 $\gamma$ is indicated by white arrow. Inset in panel B shows a 
cropped and zoomed image (scale bar $=5 \mu \mathrm{m}$ ) of large punctate structures immunoreactive for PDE6 (ranging from 0.5 to $2 \mu \mathrm{m}$ in diameter) within the inner segment. D. Rod T $\alpha$ (green) and E. Rod T $\gamma$ (green) counterstained with CNGA1 (red) in $\operatorname{Tg}(-)$ and $\operatorname{Tg}(+)$ sections. White arrow indicates mislocalized transducin in the IS.

Figure 6. Progressive accumulation of rod PDE6 and colocalization of assembled rod PDE6 with GRK1 and transducin. A. Immunolocalization of PDE6 $\beta$ in retinal cross sections of $\operatorname{Tg}(-)$ and $\operatorname{Tg}(+)$ tissues from various ages (PN20-PN50). B. PDE6 $\alpha \beta \gamma$ (identified by ROS1 antibody), rod T $\gamma$ (Top Panel: red) immunolocalization in $\mathrm{Tg}(-)$ and $\mathrm{Tg}(+)$ retinal cross sections at PN50. Zoomed image on right illustrates colocalization of PDE6 $\alpha \beta \gamma$ with T $\gamma$ with PDE6 $\alpha \beta \gamma$ in IS punctate structure indicated by white arrow (scale bar $=3 \mu \mathrm{m}$ ).

Figure 7. Accumulation of endosomal vesicles in the retina expressing ARL3-Q71L. A. Semi-thin toluidine stained retinal sections from $\operatorname{Tg}(-)$ and $\operatorname{Tg}(+)$ animals at PN30 (scale bar $=20$ $\mu \mathrm{m})$. B. Electron micrographs at low magnification illustrating the structural abnormalities of the photoreceptor OS and IS in $\operatorname{Tg}(+)$. Littermate $\operatorname{Tg}(-)$ serves as the control. Increased frequency of endosomes (white arrowhead), IS membrane whorls $\left(^{*}\right)$, and mis-oriented/dysmorphic OS discs (white arrow) (scale bar $=5 \mu \mathrm{m})$. C. Electron micrographs from $\mathrm{Tg}(+)$ retina displaying high magnification images of the structures from B. (top 3 rows) as well as dilated Golgi apparatus (black arrows) and Muller glial cells extending through the outer limiting membrane (MC) $($ scale bar $=0.5 \mu \mathrm{m})$. D. Comparison of immunofluorescent punctate structure with PDE6 $\beta$ immunoreactivity on the same scale as the high magnification electron micrographs of the abnormal structures in the IS (scale bar $=1 \mu \mathrm{m}$ ).

Figure 8. PrBPS interacts with ARL3-Q71L in vivo. Immunoprecipitation (IP) of retinal isolates from $\operatorname{Tg}(-)$, Tg(+) ARL3-WT (middle), and Tg(+) ARL3-Q71L (right) using rat 
monoclonal antibody against HA tag. Following IP, immunoblots were probed with indicated antibodies. $\mathrm{T}=$ Total, $\mathrm{U}=$ Unbound, and $\mathrm{E}=$ Eluate.

Figure 9. Defective migration of rod photoreceptor cells in ARL3-Q71L retina. A. Retinal cross sections stained for HA (red) and DAPI (blue) comparing Tg(-) littermate and Tg(+) tissues at PN25. The zoomed image below was cropped from the white dashed box in the merge image above. B. Retinal cross sections stained for rod-specific PDE6 $\beta$ (green) and DAPI (blue) comparing $\operatorname{Tg}(-)$ and $\operatorname{Tg}(+)$ tissues at PN25. The diagram below illustrates the observed migration defect in transgenic animals expressing ARL3-Q71L.

Figure 10. Mechanistic Model for mistrafficking of prenylated proteins in ARL3-Q71L mouse. A. After prenylation and further processing, PDE6 and GRK1 are peripherally bound to the ER membrane. $\operatorname{PrBP} \delta$, or its homologs, extract prenylated proteins from the endomembrane and target them to the destination membrane. ARL3 is activated by its GEF (ARL13b) in the area of the destination membrane and binds to $\operatorname{PrBP} \delta$ causing release of PDE6 and/or GRK1. RP2 accelerates the GTPase activity of ARL3 stimulating release of PrBP $\delta$ from the PrBP $\delta$ ARL3-GTP complex. B. Exogenously expressed active ARL3-Q71L sequesters PrBP $\delta$ and its homologs, thereby rendering it in capable of extracting PDE6 from the ER membrane. PDE6 and other prenylated proteins buildup in the ER membrane and are pushed out of the ER in endosomal vesicles which accumulate in the IS. 


\section{ABBREVIATIONS}

ARL3 = ADP-Ribosylation Factor-Like 3

ARL3-Q71L = transgenic dominant active ARL3

ARL3-WT = transgenic wild-type ARL3

RP2 = Retinitis Pigmentosa Protein 2

PDE6 $=$ Phosphodiesterase 6

PDE6 $\alpha=$ phosphodiesterase, alpha subunit

PDE6 $\beta=$ phosphodiesterase, beta subunit

PDE $\gamma=$ phosphodiesterase, gamma subunit

GRK1 = Rhodopsin Kinase (G-protein receptor kinase 1)

$\mathrm{RP}=$ Retinitis pigmentosa

$\mathrm{XLRP}=\mathrm{X}$-linked recessive retinitis pigmentosa

DAPI = 4',6-diamidino-2-phenylindole

$\mathrm{ERG}=$ electroretinogram

OS = outer segment

ROS $=$ rod outer segment

COS = cone outer segment

IS = inner segment

RIS $=$ rod inner segment

CIS = cone inner segment

$\mathrm{ONL}=$ outer nuclear layer

$\mathrm{INL}=$ inner nuclear layer

CNGA1 = cyclic nucleotide gated channel 
Figure 1

A marl3 Q71L

\begin{tabular}{|c|c|c|}
\hline Rhop \\
\hline
\end{tabular}

B

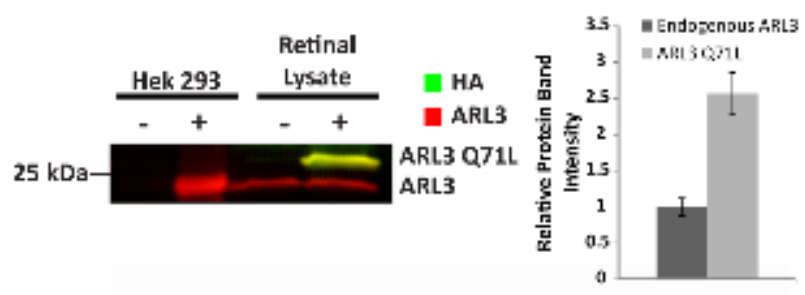

D
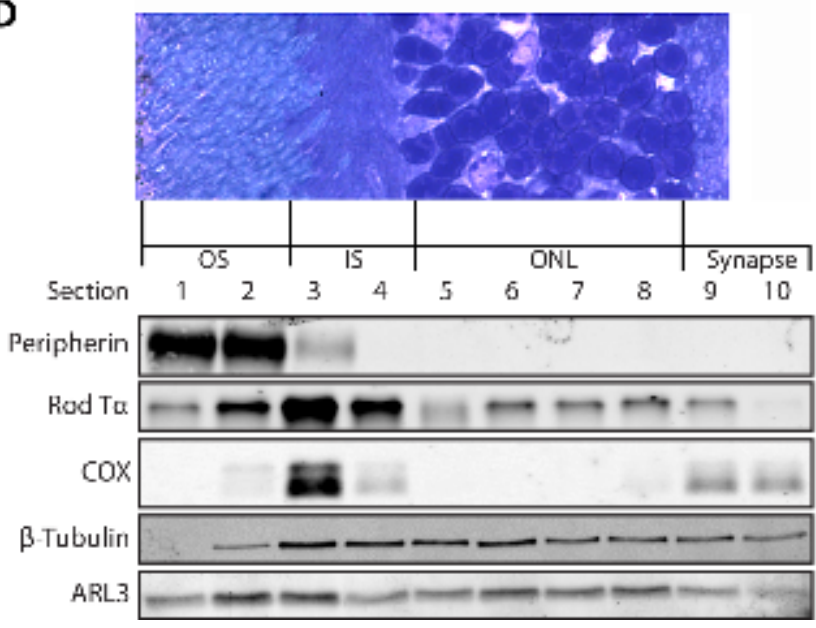

C
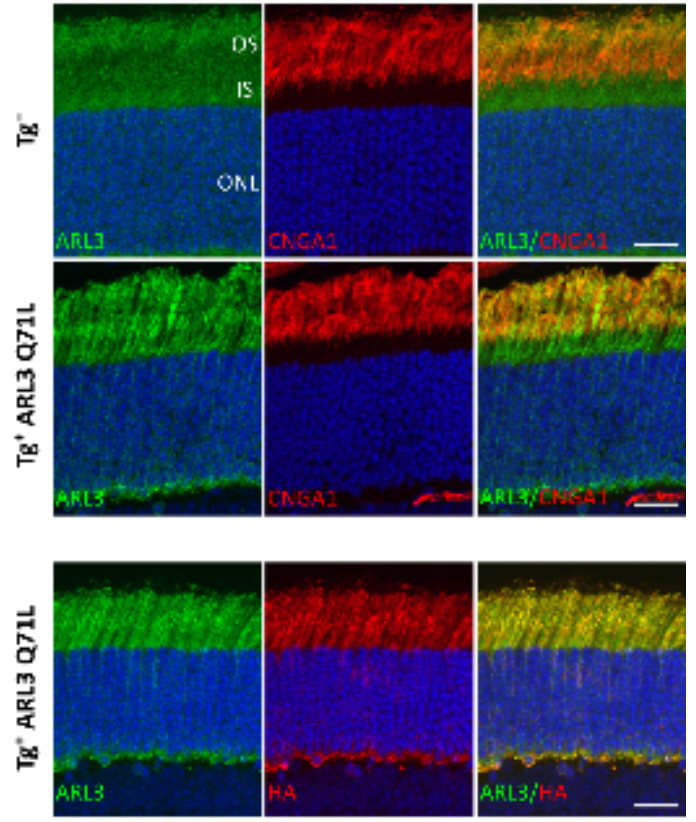
Figure 2

A

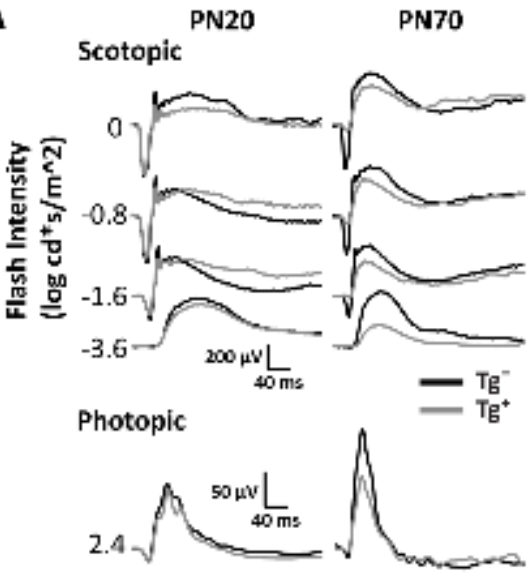

C

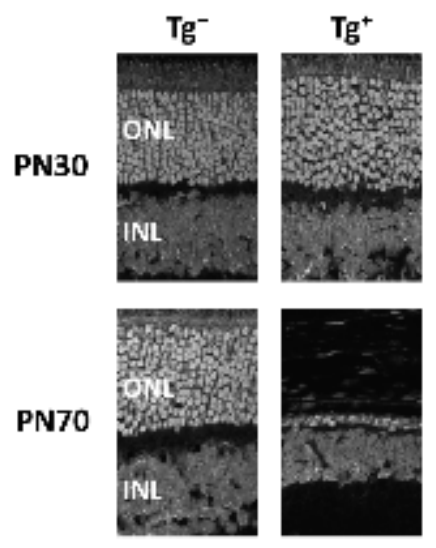

B

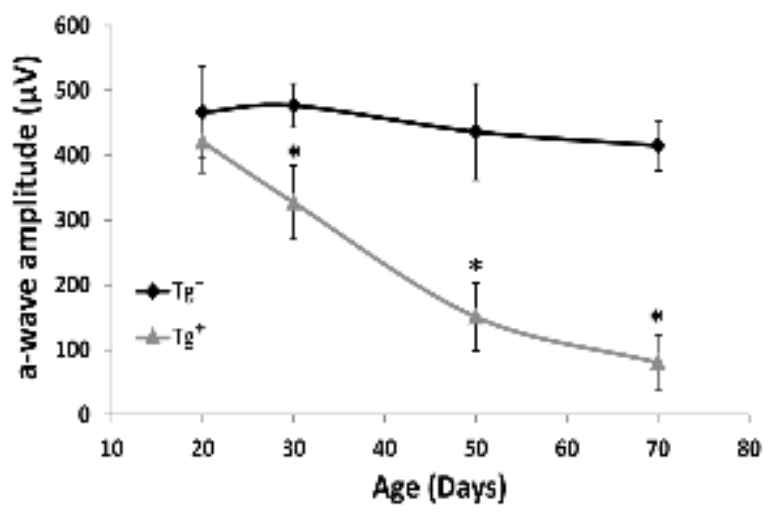

D

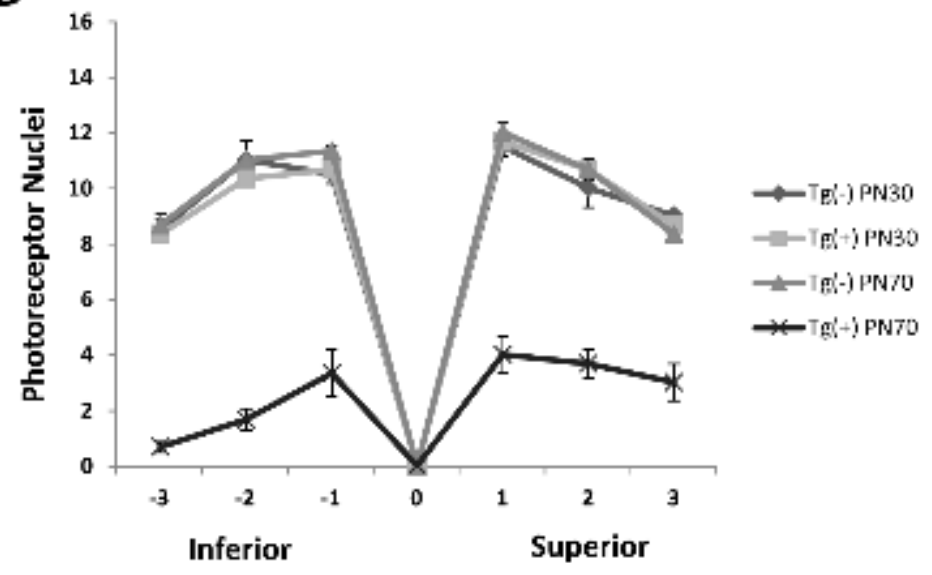


Figure 3

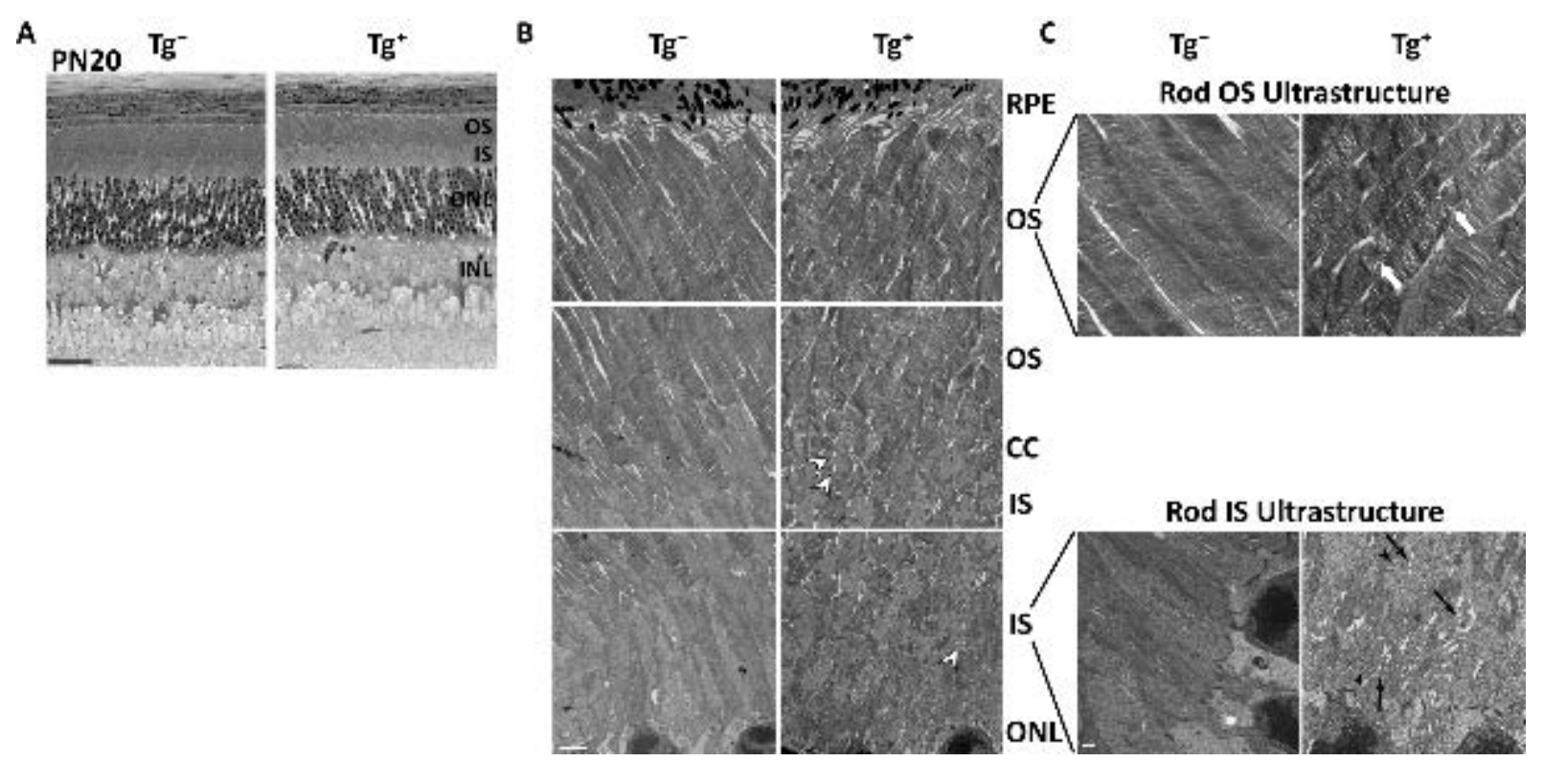


Figure 4
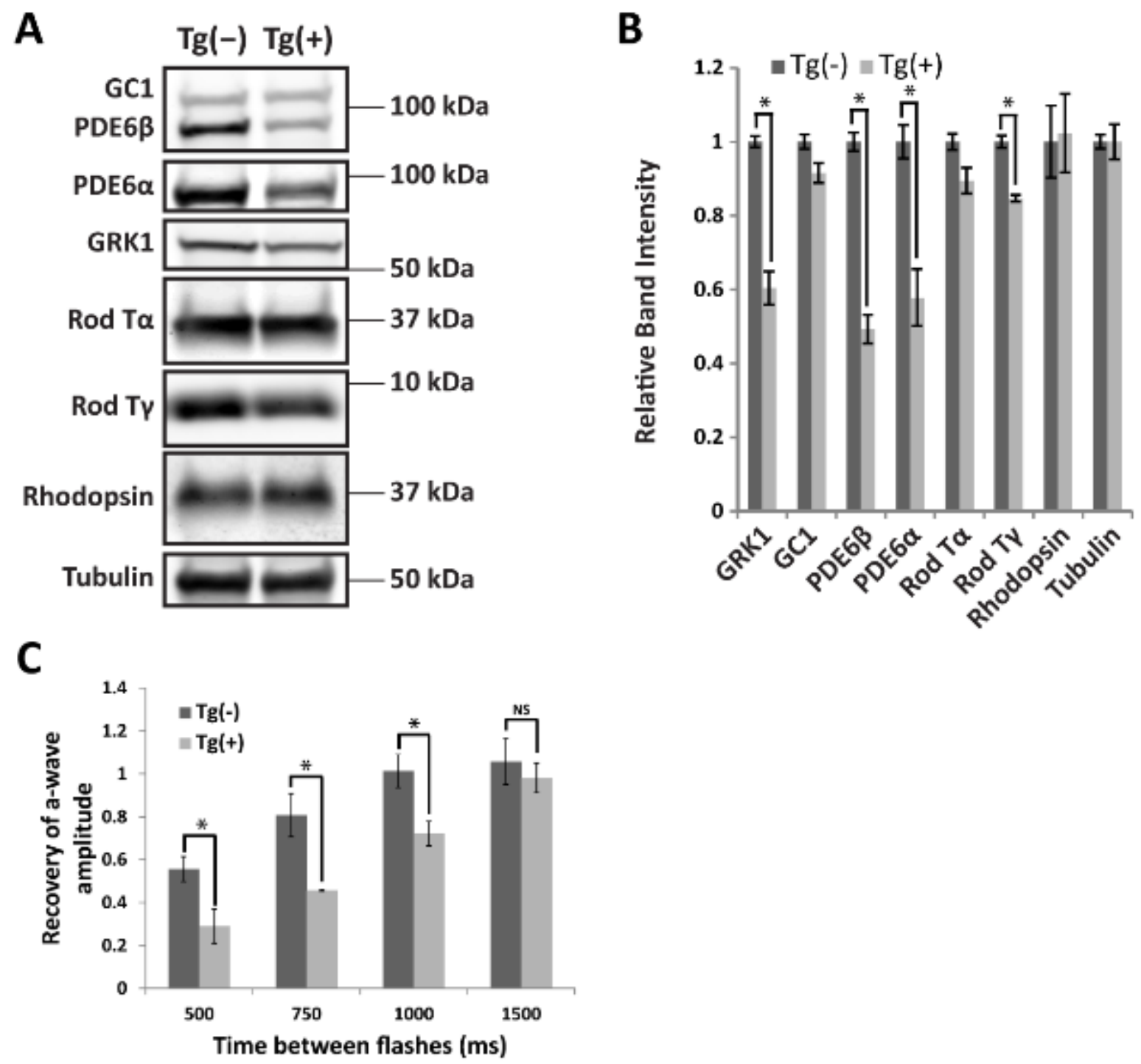
Figure 5

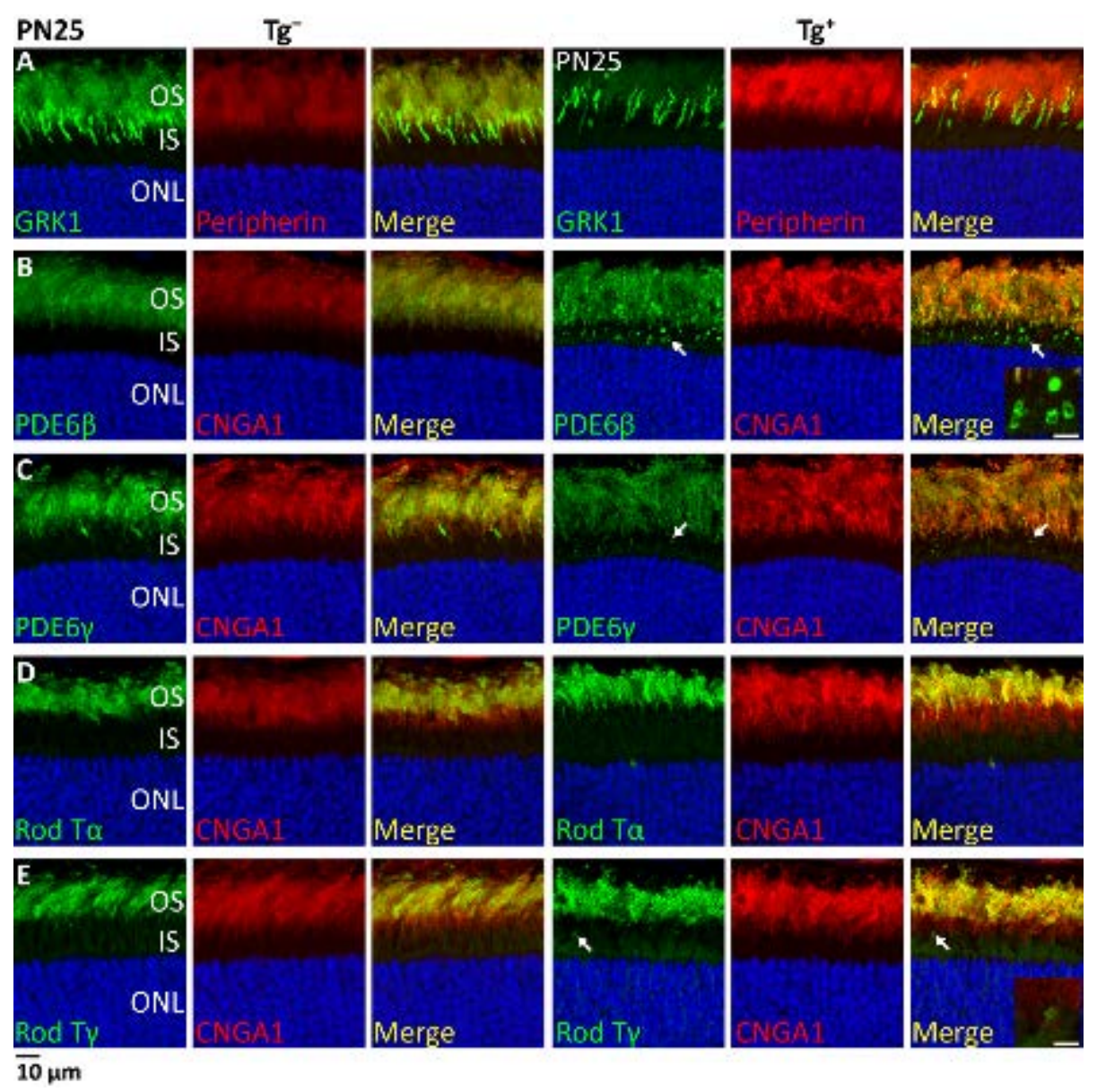


Figure 6
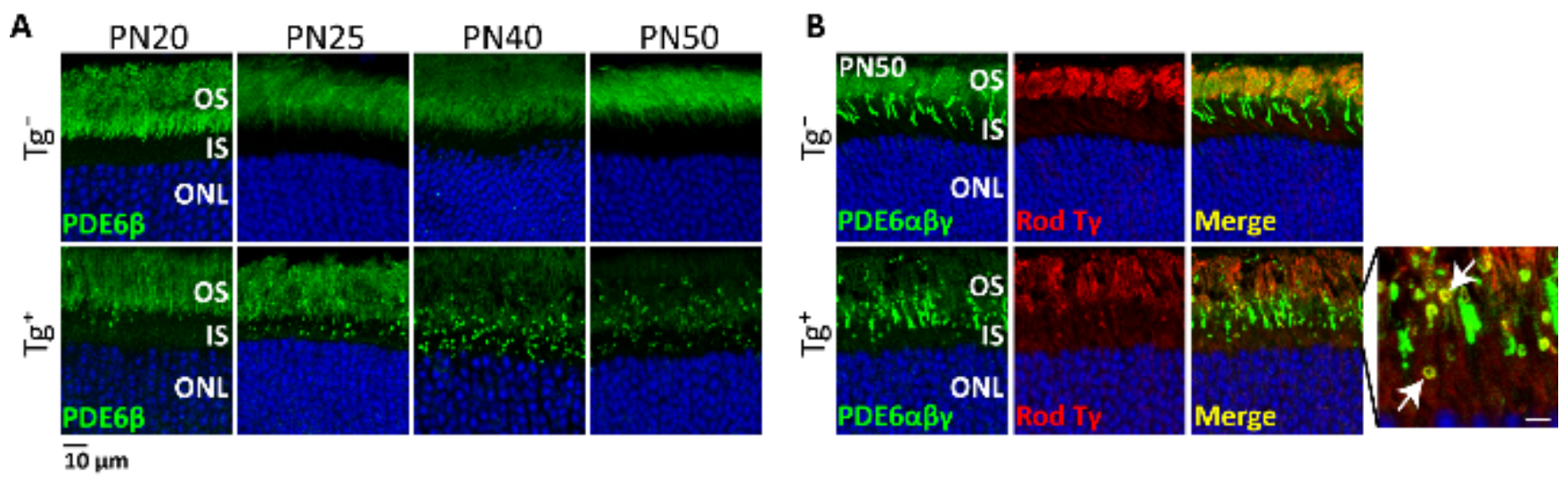
Figure 7
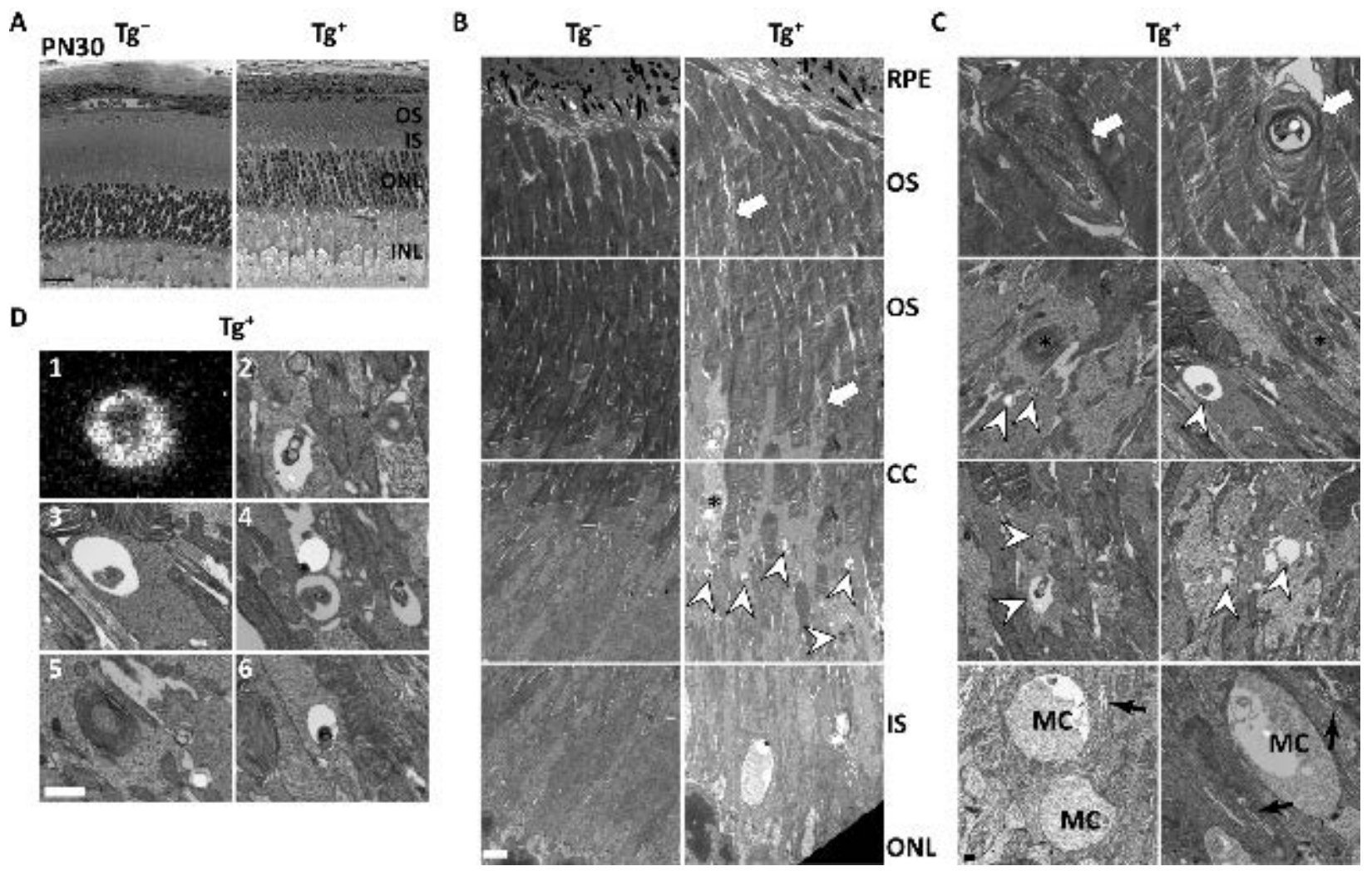
Figure 8

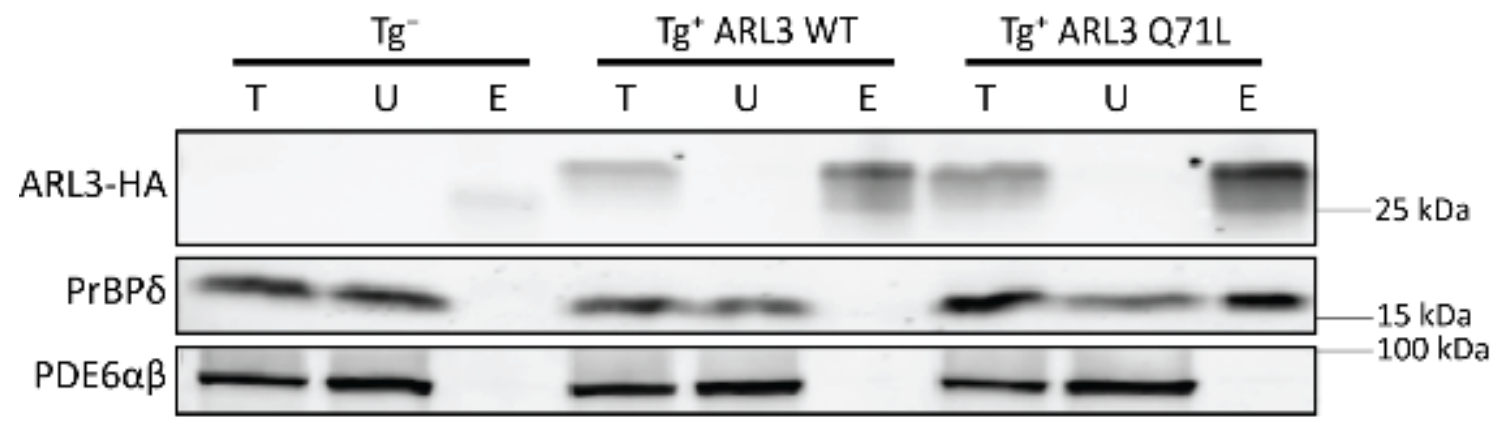




\section{Figure 9}
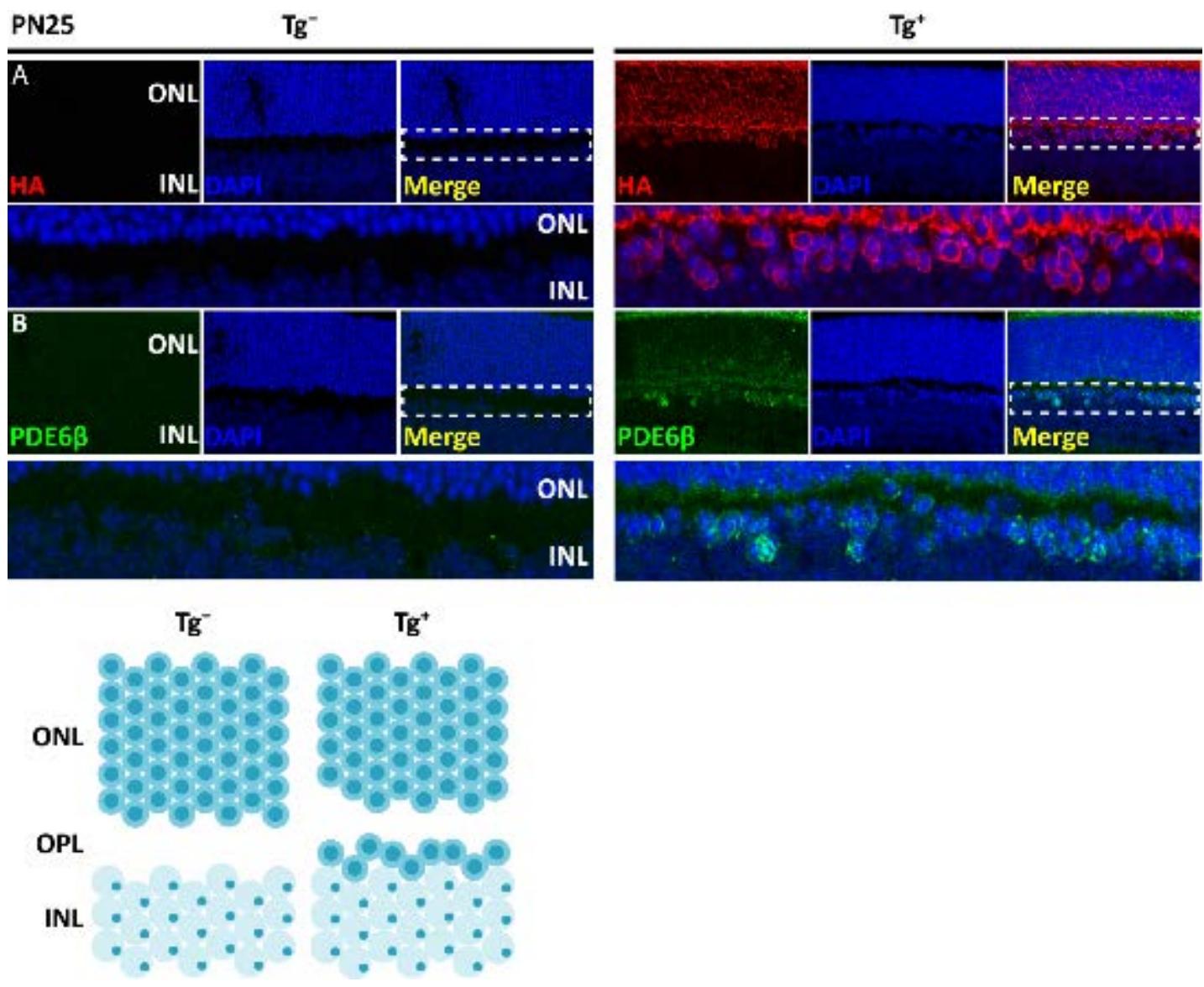
Figure 10

A

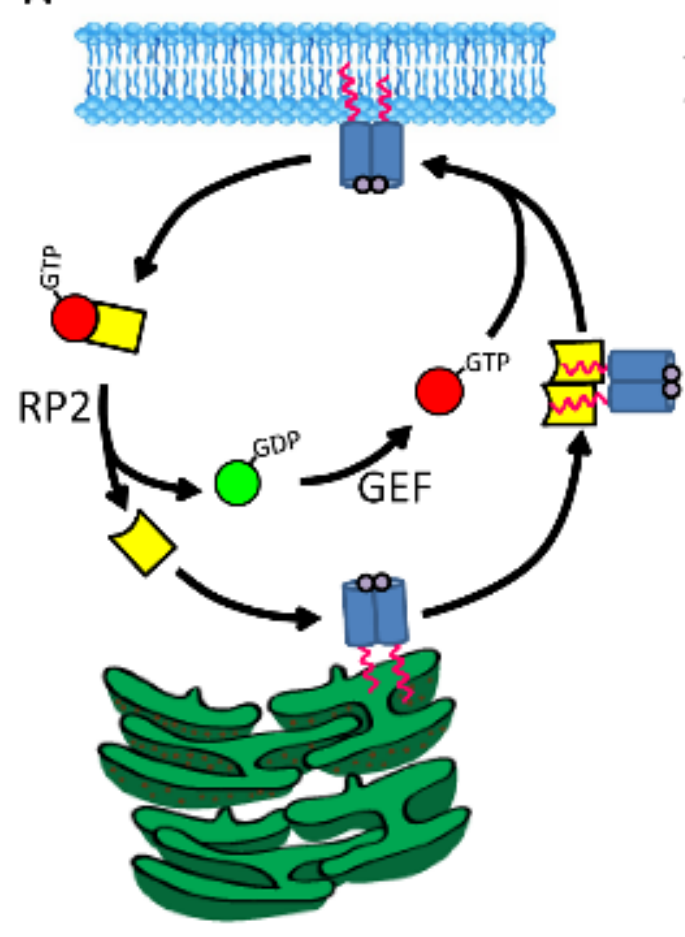

B

Destination

Membrane

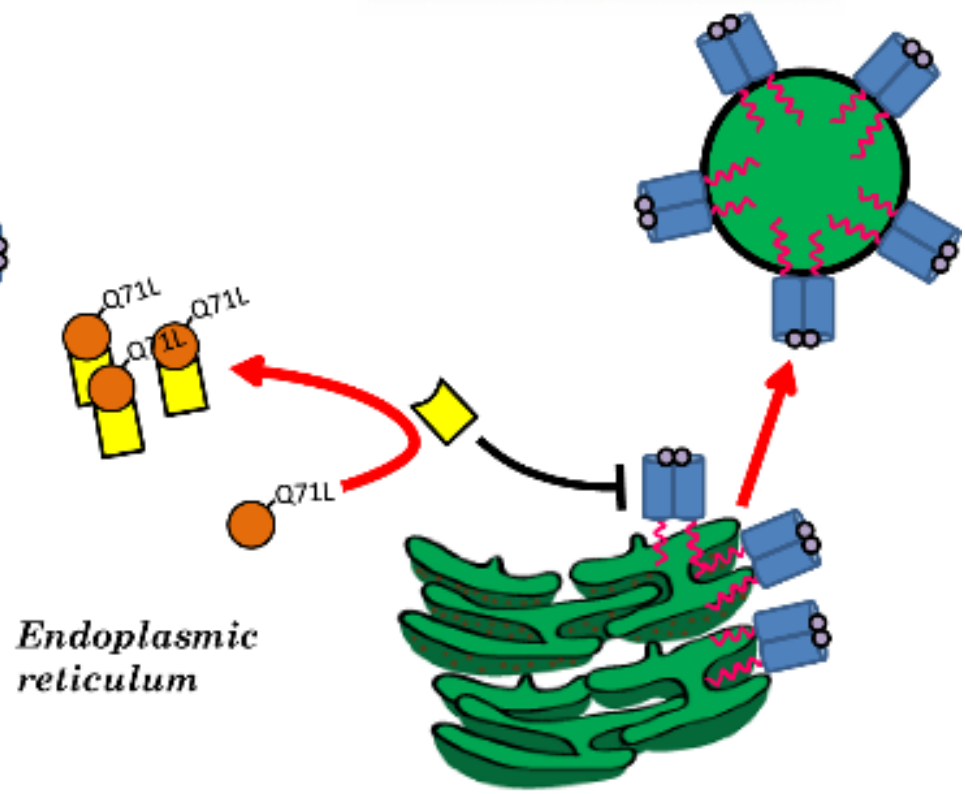

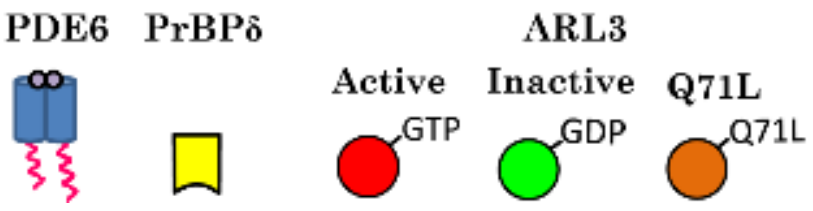




\section{Chapter 3:}

ADP-Ribosylation Factor-Like 2 (ARL2) regulates ciliogenesis and development of outer segments in rod photoreceptor neurons.

\section{Zachary C. Wright ${ }^{1 a}$, Daniel Murphy, Peter Stoilov, Andrew F.X. Goldberg², and Visvanathan Ramamurthy ${ }^{1 \mathrm{a}, \mathrm{b}, \mathrm{c} *}$}

Departments of a Ophthalmology and ${ }^{\mathrm{b}}$ Biochemistry, ${ }^{\mathrm{c}}$ Center for Neuroscience, Robert C. Byrd Health Sciences Center, ${ }^{1}$ West Virginia University, Morgantown, West Virginia 26506, USA;

${ }^{2}$ Eye Research Institute, Oakland University, Rochester, Michigan, 48309, USA

*Address Correspondence to: Visvanathan Ramamurthy, West Virginia University Eye Institute, One Medical Drive, E-363, Morgantown, WV 26506-9193, Tel: 304598 6940; Fax: 304-5986928; Email: ramamurthyv@wvumedicine.org 


\section{ABSTRACT}

Photoreceptor cells are specialized neurons that contain an elaborate membrane structure, the outer segment (OS), associated with a non-motile cilium. Many diseases result in the malformation of the OS and these diseases often occur because of an inherited defect in the genes involved in ciliogenesis. ADP-ribosylation factor-like 2 (ARL2) has recently been implicated in OS formation through its association with ARL2 binding partner (ARL2BP), a protein linked to inherited blinding disease. We have developed a transgenic mouse model expressing a tagged-dominant active form of human ARL2 (ARL2-Q70L) under a rod-specific promoter. Transgenic ARL2-Q70L animals exhibit significant decreased photoreceptor cell function as early as post-natal day 16 (P16) and progressive rod degeneration. Furthermore, ARL2-Q70L expression results in misdevelopment of inner and outer segments associated with mislocalization of rhodopsin. The stunted outer segments are attributed to the observed decrease in axonemal length. Additionally, immunofluorescence and ultrastructural analysis revealed aberrantly localized photoreceptor cilia at the base of the inner segment. Finally, immunoprecipitation revealed interaction of ARL2-Q70L with known tubulin chaperone protein, tubulin binding cofactor D (TBCD), suggesting a potential role for tubulin dysregulation in these cells. In conclusion, ARL2-Q70L is crucial for photoreceptor neuron sensory cilium development and it specifically functions to regulate the process of ciliogenesis. 


\section{INTRODUCTION}

Photoreceptor neurons utilize the outer segment (OS), a specialized non-motile cilium, to efficiently capture and transmit light signal (Pearring et al., 2013). The development of a functional OS with stacked membrane discs packed with phototransduction and structural proteins depends on cilia formation, i.e. ciliogenesis. A number of diseases result from malformation of the OS and these diseases frequently occur because of an inherited defect in the genes involved in ciliogenesis. These defects result in blinding disease as well as syndromic disorders known as ciliopathies, emphasizing the importance of cilia in development and functioning of multiple organs (Goetz and Anderson, 2010; Mockel et al., 2011).

In order to gain insight into the development of OS and the related diseases, it is crucial to identify the proteins involved in this process. Interestingly, there are a number of small GTPases, which act as molecular switches, that are believed to play a role in regulating proteinprotein interactions throughout the process of ciliogenesis and OS formation ( $\mathrm{Li}$ and $\mathrm{Hu}, 2011$; Li et al., 2012; Pearring et al., 2013; Zhang et al., 2013). These proteins are likely important for temporal and spatial regulation of ciliary proteins, thus adding a level of complexity to the series of events necessary for photoreceptor morphogenesis. More specifically, the protein ADPribosylation factor-like 2 (ARL2) has been implicated in the process of OS formation in photoreceptor cells through its suspected interaction of the disease-linked gene, ARL2 Binding Protein (ARL2BP). Importantly, ARL2, as is the case with many small GTPase effector proteins, selectively interacts with ARL2BP in the active state (i.e. ARL2-GTP). ARL2BP is necessary for retinal function in humans as mutations in this protein result in the blinding disease, retinitis pigmentosa (RP) (Davidson et al., 2013). Moreover, it is hypothesized that ARL2BP-linked RP is likely the result of loss of interaction with ARL2. 
There is a large gap in knowledge concerning the role of ARL2 in photoreceptor neurons as well as in vivo function of this protein in general. Studies of ARL2 and ARL3, an ARL2 homolog, suggest that they may have overlapping functions. Specifically, they are thought to be regulators of prenylated protein trafficking through their interaction with prenylated binding protein $\delta$ (PrBP $\delta$ ) (Ismail et al., 2011; Wätzlich et al., 2013). This function of ARL3 has been shown in vivo by multiple animal models including dominant active transgenic ARL3-Q71L and conditional ARL3 knockout mice (Hanke-Gogokhia et al., 2016; Wright et al., 2016). However, a role for ARL2 in prenylated protein trafficking has yet to be tested in vivo.

Although it has not been specifically identified as a connecting cilium protein in photoreceptor cells, ARL2 has been implicated in a number of cellular processes (Kahn et al., 2005; Zhou et al., 2006; Tian et al., 2010; Ismail et al., 2011; Taniuchi et al., 2011; Newman et al., 2014). Specifically, ARL2 is thought to play a role in microtubule formation and regulation of the soluble tubulin pool (Bhamidipati et al., 2000; Burd et al., 2004; Zhou et al., 2006; Tian et al., 2010; Nithiananatham et al., 2015). A number of studies have shown that expression of dominant active ARL2 causes microtubule destruction by preventing polymerization (Bhamidipati et al., 2000; Zhou et al., 2006; Tian et al., 2010). More recent studies have shown that active ARL2-GTP appears to interact with tubulin binding cofactors D and E (i.e. TBCD and TBCE) to form a complex that can regulate the state of tubulin dimerization and therefore the pool of free polymerizable tubulin (Nithiananatham et al., 2015). Furthermore, knockdown of ARL2 or its binding partner ARL2BP results in shortening of cilia in ARPE-19 cell lines (Davidson et al., 2013). Furthermore, it is important to note that ARL2 is localized at the centrosome in cell lines where it is proposed to act as a microtubule regulator in an ARL2BP- 
independent manner (Zhou et al., 2006). These data suggest that ARL2 may play a role in ciliogenesis by regulating tubulin or ARL2BP at photoreceptor cilium.

The purpose of this study was to gain insight into the role of ARL2 in rod photoreceptor cells by expression of the dominant active mutant ARL2-Q70L. Here we show for the first time that proper regulation of ARL2 is necessary for rod ciliogenesis and OS formation. Additionally, we show that ARL2 interacts with tubulin binding cofactor D (TBCD), a key player in the regulation of tubulin to form microtubules. 


\section{RESULTS}

\section{Endogenous Arl2 Expression Profile and Dominant Active Mutant Transgenic Model}

\section{Generation}

In order to investigate the role of ARL2 in mouse photoreceptor cells, it is important to understand the temporal dynamics of Arl2 gene expression. We examined the developmental retinal mRNA expression profile of endogenous Arl2 and Arl3 in mice (Figure 1A). Real-time PCR was performed in order to quantitate the message levels of these genes at post-natal day 0 (P0) through P16. As shown in the figure, Arl2 expression remains low until a 4-fold spike in message between ages P6 and P9. This “switch” in expression suggests that there is functional need for ARL2 corresponding to elaboration of the OS. Arl3 expression showed a similar pattern, exhibiting a moderate change in expression at P6-P9.

To determine the role of ARL2 in photoreceptor function, we generated a transgenic animal expressing dominant active human ARL2-Q70L in rod photoreceptor cells (Fig. 1B). Expression of ARL2-Q70L is under the control of a rhodopsin promoter (Rhop) that initiates the expression of transgenes as early as post-natal day 4 (P4) in rod photoreceptor cells of the retina (Song et al., 2014). This approach is similar to our recently published work where we expressed ARL3 in rod photoreceptor cells (Wright et al., 2016). Transgenic founders were crossed with 129/SV-E wild-type mice for a few generations to ensure consistent transgene inheritance and expression prior to analysis. Transgenic negative littermates of ARL2-Q70L mice were used as control animals for comparison unless stated otherwise.

We generated an affinity purified rabbit antibody against ARL2. This antibody specifically recognized ARL2 and not its homologue ARL3 expressed in Human Embryonic 
Kidney (HEK293) cells (data not shown). In retina from transgenic animals, we find that ARL2Q70L ( 23 kDa) was expressed at 40\% the level of endogenous ARL2 ( 20 kDa) protein (Fig. 1C; $\mathrm{p}<0.001$ ). Additionally, an anti-HA antibody was used to confirm the identity of the tagged ARL2-Q70L (Fig. 1C (green)).

The expression of transgenic ARL2-Q70L was confirmed by indirect immunofluorescence with anti-HA antibody (Fig. 1D). ARL2-Q70L was found in throughout rod photoreceptor cells except for the OS. Similar to endogenous ARL2 proteins, we observed transgene expression in the photoreceptor connecting cilium (Fig. 1D-E). Unlike ARL3, endogenous and transgenic ARL2 are excluded from the rod OS (Wright et al., 2016) (Fig. 1DE).

Overall, these data show a switch in endogenous Arl2 expression between P6 and P9 coinciding with the early stages of photoreceptor OS elaboration (Obata and Usukura, 1992) and that it is localized primarily to the connecting cilium region.

\section{Decreased photoreceptor function in ARL2-Q70L mice}

We performed electroretinography (ERG) starting from P16 to determine the impact of expression of dominant active ARL2 on rod photoreceptor cell function. Transgenic ARL2Q70L animals exhibited a dramatic $~ 70 \%$ reduction in scotopic a-wave at P16 compared to transgenic littermate controls which showed a robust a-wave and b-wave (Fig. $2 \mathrm{~A}$; $\operatorname{Tg}(-)=379.8$ $\pm 7.2 \mu \mathrm{V}$ and $\operatorname{Tg}(+)=120.0 \pm 3.3 \mu \mathrm{V}, \mathrm{p}<0.0001)$. As expected, cone photoreceptor response remained unaffected at this time point as transgenic negative and transgenic positive photopic bwaves were comparable (Fig. 2A; $\operatorname{Tg}(-)=123.18 \pm 6.4 \mu \mathrm{V}$ and $\operatorname{Tg}(+)=138.2 \pm 1.9 \mu \mathrm{V} ; \mathrm{p}=$ 0.17). Rod photoresponse progressively declined in transgenic ARL2-Q70L animals with an 
85\% reduction in a-wave by P100 compared to littermate controls (Fig. 2A-B; $\operatorname{Tg}(-)=379.3 \pm$ $19.6 \mu \mathrm{V}$ and $\operatorname{Tg}(+)=60.7 \pm 9.7 \mu \mathrm{V}, \mathrm{p}<0.0001)$. Finally, only a slight difference rod sensitivity was noted with scotopic a-wave half-saturating light intensities of $0.010 \pm 0.0019 \mathrm{~cd} * \mathrm{~s} / \mathrm{m}^{2}$ in $\mathrm{Tg}(+)$ animals versus $0.014 \pm 0.0018 \mathrm{~cd} * \mathrm{~s} / \mathrm{m}^{2}$ in littermate controls and calculated maximum awave amplitudes of $225.2 \pm 6.6 \mu \mathrm{V}(\operatorname{Tg}(+))$ and $499.7 \pm 10.6 \mu \mathrm{V}(\mathrm{Tg}(-))$ (Supplementary Fig. 1). Taken together, these results show that expression of ARL2-Q70L reduced rod photoreceptor function that declined over time.

\section{Rod photoreceptor cells degenerate in animals expressing ARL2-Q70L}

In order to determine if the reduction in photoreceptor function was due photoreceptor cell death, we investigated the outer nuclear layer (ONL) length from P13 to P110. At P13, there was no difference in the size of the ONL (Fig. 3A-B; $\operatorname{Tg}(-)=11.0$ layers versus $\operatorname{Tg}(+)=10.2$ layers; $\mathrm{p}=$ 0.26), however, there was a noticeable increase in the number of apoptotic nuclei observed with propidium iodide. At P16 apoptotic nuclei were more frequent with a minor decrease in the ONL length. (Fig. 3A, arrows indicate apoptotic nuclei). By P60 there was a major loss of photoreceptor cells and by P110 the ONL was nearly gone with only 1-3 nuclear layers remaining (Fig. 3A-B; P110: $\operatorname{Tg}(-)=10.2$ layers and $\operatorname{Tg}(+)=2.0$ layers; $\mathrm{p}<0.01$ at all locations). These data suggest that defects during development of rod photoreceptor cells results in progressive degeneration.

\section{Reduction of photoreceptor OS proteins ARL2-Q70L animals}

Loss of photoresponse in ARL2-Q70L animals at P16 does not correlate to the minor loss of cells at that age. This suggests a defect in phototransduction cascade or a reduction in levels of phototransduction proteins. Previous in vitro studies have implicated ARL2 as a regulatory 
protein for prenylated cargo through its interaction with $\operatorname{PrBP} \delta$ similar to its homolog ARL3 (Ismail et al., 2011; Wätzlich et al., 2013). Therefore, we investigated the levels of prenylated phototransduction proteins along with other non-prenylated proteins in ARL2-Q70L expressing animals prior to degeneration (Fig. 4). The prenylated proteins phosphodiesterase $6 \alpha$ (PDE6 $\alpha$ ) and $\beta$ (PDE6 $\beta$ ) subunits were significantly reduced by $\sim 30 \%$ at $\mathrm{P} 13$ compared to transgenic negative littermates (PDE6 $\alpha$ : P13 $\approx-30 \% \mathrm{p}<0.001$; PDE6 $\beta$ : P13 $\approx-34 \% \mathrm{p}<0.01$ ). Similarly, farnesylated G-protein coupled receptor kinase 1 (GRK1) and rod transducin $\gamma$ subunit (G $\gamma 1)$ as well as myristoylated rod transducin $\alpha$ (Gat1) were all significantly reduced at the P13 (GRK1: $\mathrm{P} 13 \approx-17 \% \mathrm{p}<0.01 ; \mathrm{G} \gamma 1: \mathrm{P} 13 \approx-50 \% \mathrm{p}<0.01 ; \mathrm{G} \alpha \mathrm{t} 1: \mathrm{P} 13 \approx-11 \% \mathrm{p}<0.01)$. However, to our surprise, the non-prenylated OS proteins guanylate cyclase 1 (GC1), rhodopsin, and peripherin were all significantly reduced by approximately the same magnitude as the prenylated proteins at both P13 (GC1: P13 $\approx-31 \% \mathrm{p}<0.05$; rhodopsin: $\mathrm{P} 13 \approx-51 \% \mathrm{p}<0.001$; peripherin: $\mathrm{P} 13 \approx-30 \%$ $\mathrm{p}<0.05$ ). A similar pattern was seen in P16 retinal samples as well (Supplementary Fig. 2). These proteins were all normalized to $\mathrm{G} \alpha_{0}$, which is expressed in the bipolar cells and was unchanged between transgenic and littermate controls $(\mathrm{P} 13 \mathrm{~s} \mathrm{p}=0.33)$. Additionally, tubulin was unchanged between transgenic animals and controls $(\mathrm{P} 13 \mathrm{p} \mathrm{p}=0.99)$. These results are in contrast to mouse models disrupting the ARL2 homolog, ARL3, which result in specific loss of prenylated proteins prior to cell death (Hanke-Gogokhia et al., 2016; Wright et al., 2016). This pan-decrease in photoreceptor OS proteins suggests that ARL2 is not acting specifically through $\operatorname{PrBP} \delta$ to disrupt prenylated proteins, but is actually functioning through a broader mechanism.

\section{Abnormal elaboration of photoreceptor OS in ARL2-Q70L Mice}

Because there is a pan-decrease in OS protein levels with no major loss in photoreceptor cells at P13, it is likely that there is a structural defect of the OS. For initial insight into 
photoreceptor cell structure, we examined morphology via light microscopy using toluidine blue staining. We completed this at P13 and P16 before ONL degeneration and after initial significant photoreceptor loss, respectively. Transgenic ARL2-Q70L had a significant 2-fold decrease in the OS length at P13 (Fig. 5; P13: $\operatorname{Tg}(-)=10.9 \pm 1.1 \mu \mathrm{m}$ versus $\operatorname{Tg}(+)=4.9 \pm 0.5, \mathrm{p}<0.05$ ). Furthermore, there was a significant 1.7-fold decrease in IS length in $\mathrm{Tg}(+)$ at P13 (Fig. 5; P13: $\operatorname{Tg}(-)=12.7 \pm 0.1 \mu \mathrm{m}$ versus $\operatorname{Tg}(+)=7.4 \pm 0.2 \mu \mathrm{m}, \mathrm{p}<0.001)$. Additionally, there were no significant differences in ONL length between transgenic negative and positive at P13 (Fig. 5; P13: $\operatorname{Tg}(-)=53.2 \pm 2.7 \mu \mathrm{m}$ versus $\operatorname{Tg}(+)=52.3 \pm 1.6 \mu \mathrm{m}, \mathrm{p}=0.79)$. Similar results were found at P16 except there was a minor, but significant reduction in the ONL length at this age. These data further support the conclusion that transgenic expression of ARL2-Q70L results in a development of photoreceptors.

\section{Mislocalization of Rhodopsin in ARL2-Q70L animals}

Structural defects in the OS (such as loss of the connecting cilium or decreased OS length) result in mislocalization of phototransduction proteins such as rhodopsin (Jiang et al., 2015; HankeGogokhia et al., 2016). We wanted to determine the effect of stunted OS structure on the localization of OS proteins. Additionally, the ARL2 homolog, ARL3, has be shown to regulate trafficking of prenylated proteins to the rod OS (Hanke-Gogokhia et al., 2016; Wright et al., 2016). Therefore, we also wanted to determine if ARL2 serves a redundant function to ARL3 in this process. To answer these questions, we examined rod OS proteins using immunofluorescence microscopy at P13 and P16 (Fig. 6). Of note, rhodopsin was dramatically mislocalized to the ONL surrounding the nuclei as well as the IS and synapse at P13 and P16 in ARL2-Q70L animals (Fig. 6 \& Supplementary Fig. 3, Panel B). Immunoreactivity for PDE6 and rod transducin was also found in the IS, ONL, and synapse similar to rhodopsin albeit to a lesser 
extent at P13 and P16 (Fig. 6, Panel A and C and Supplementary Fig. 2, Panel A and C). Finally, GRK1 showed normal localization in P13 animals, but exhibited decreased immunoreactivity in the rod OS with minor mislocalization at the synapse at P16 (Supplementary Fig. 2, Panel D). Unlike opsin, the prenylated proteins GRK1, PDE6, and rod transducin localized normally. Our results are also in agreement with previous studies that show that structural defects of the OS result in mislocalization of rhodopsin.

\section{ARL2-Q70L expression results in defects in ciliogenesis and OS development in rod photoreceptor cells}

ARL2 has recently been implicated in the regulation of ciliary length in vitro (Davidson et al., 2013). To explore this potential role in intact photoreceptor cells, immunofluorescence and transmission electron microscopy was performed to study axonemal integrity and rod photoreceptor cell ultrastructure. Utilizing RP1 and RPGR immunoreactivity the length of the connecting cilium and axoneme was measured in P13 ARL2-Q70L retina (Fig. 7A). Immunoreactivity of these two markers in control $\operatorname{Tg}(-)$ retina was as expected with uniform staining generating a clear IS-OS border with 1 to $2 \mu \mathrm{m}$ connecting cilium extending a $2-4 \mu \mathrm{m}$ axoneme (Fig. 7A; Top Panel). However, in transgenic animals $(\operatorname{Tg}(+))$ the IS-OS border is less apparent with a number of instances of cilium staining within the IS region near the outer limiting membrane (OLM) (Fig. 7A; Bottom Panel; White Arrows). Although the length of the connecting cilium, as measured by RPGR immunoreactivity is comparable to wildtype tissue, the length of the axoneme (measured by RP1 immunoreactivity) is dramatically shorter (Fig. 7A; Bottom Panel). More specifically, ciliary length in $\mathrm{Tg}(+)$ animals was on average $\sim 2$-fold shorter than littermate controls with approximately $70 \%$ of cilia between 1 and $2 \mu \mathrm{m}$ compared to the same proportion between 3 and $4 \mu \mathrm{m}$ (Fig. 7A; Right). 
In order to explore ultrastructural defects, electron microscopy was performed at P13 when there was an observed decrease in IS and OS length, but prior to significant cell death. Photoreceptor OSs were frequently shorter and misoriented in comparison to wildtype controls (Fig. 7B-C; White Arrowheads). Additionally, aberrantly localized cilia were found protruding from the lower portions of the IS near the OLM (Black Arrows) in the retina from transgenic positive animals. In contrast there is a clear demarcation between the inner and outer segment in controls (Fig. 7B-C). Similar to our results from light microscopy, the IS was dramatically shorter. Overall, these results suggest that expression of ARL2-Q70L significantly disrupts rod photoreceptor development and specifically, the development of ciliary axoneme and the OS.

\section{Tubulin binding cofactor D (TBCD) interacts with ARL2 in vivo}

The photoreceptor axoneme is a microtubule-based organelle that is required for proper cell function. Previous studies have shown that ARL2 plays a role in the regulation of the pool of tubulin dimers that are available to form microtubules (Nithiananatham et al., 2015). Additionally, expression of dominant active ARL2 in vitro causes destruction of microtubules by acting on polymerization (Zhou et al., 2006). To provide mechanistic insight into the defects seen in the ARL2-Q70L transgenic animals, we performed co-immunoprecipitation (co-IP) experiments specifically looking at candidate proteins involved in ARL2-mediated tubulin regulation. We utilized the HA tag to pull-down ARL2-Q70L protein in retinal lysates from transgenic animals. Based on in vitro binding data, we expected ARL2-Q70L to interact with a number of proteins based on previous including ARL2BP, $\alpha$ - and $\beta$-tubulin, tubulin binding cofactor D (TBCD) and TBCE (tubulin-specific chaperones), and potentially $\operatorname{PrBP} \delta$ (Bhamidipati et al., 2000; Ismail et al., 2011; Davidson et al., 2013; Wätzlich et al., 2013; Nithiananatham et al., 2015). Surprisingly, ARL2BP, $\alpha$-tubulin, and $\beta$-tubulin were undetectable 
in eluates of IP using transgenic lysates. However, TBCD was detected in the ARL2-Q70L eluate demonstrating that TBCD and ARL2-Q70L interact in vivo. 


\section{DISCUSSION}

A full understanding of the components involved in morphogenesis of the rod outer segment is lacking. In this study, we have discovered a novel regulator of rod photoreceptor development and function, ARL2. Our first indication that ARL2 plays a role in OS morphogenesis was endogenous mRNA expression profile, which demonstrates a "switch"-like spike in message levels in the retina at approximately P9, a critical period of OS elaboration (Fig. 1A). Furthermore, we observed localization of endogenous ARL2 at the connecting cilium via immunofluorescence microscopy. In order to study ARL2 in rod photoreceptor neurons, we generated a dominant active transgenic mouse model that expresses ARL2-Q70L mutant under a rod promoter (Rhop). It should be noted that the expression of our transgene follows a similar pattern to expression of native protein with transgenic protein detected at P9 (Wright and Ramamurthy, unpublished data). Expression of the transgenic protein was $40 \%$ the level of endogenous ARL2 and the total amount of ARL2 (transgenic + endogenous) is equal between the transgenic positive and transgenic negative animals. With this model, we demonstrate that expression of ARL2-Q70L results in early defects in OS development followed by slow photoreceptor degeneration.

We have verified the phenotype in multiple transgenic founder lines that show variable levels of severity (Wright and Ramamurthy, unpublished data). Importantly, the phenotype is distinct from that of any of the ARL3-Q71L lines generated for previous work (Wright et al., 2016). Specifically, ARL3-Q71L transgenic animals exhibit normal photoreceptor elaboration and function at P20 with a rapid decline in function between ages of P30 and P70 with concomitant degeneration of rods by P70. ARL2-Q70L transgenic animals have a decrease in the length of the photoreceptor OS and IS as early as P13 and abnormal rod function at P16 with a 
slow decline and degeneration to P100. Previous studies suggest that ARL2 may act similarly to ARL3 as a selective regulator for prenylated protein trafficking (Ismail et al., 2011; Wätzlich et al., 2013; Wright et al., 2016). However, we observed minor mislocalization of prenylated proteins to the ONL and synapse, and we attribute this mislocalization to stunted OS. This is in contrast to ARL3-Q71L transgenic animals, which show progressive mislocalization of PDE6 and other prenylated proteins with no effect on OS elaboration. Immunoblotting from ARL2Q70L retina shows a uniform decrease in the levels of OS proteins and not a selective decrease in prenylated proteins as occurs in ARL3-Q71L transgenic retina. Although we cannot completely rule out that ARL2 may perform act in prenylated protein trafficking, we can say that it does not appear to be its primary role in vivo. These data not only serve as a control for the effect of a transgene, but also demonstrate a novel role for ARL2 in rod photoreceptor cells that is distinct from its homolog ARL3.

Rhodopsin is mislocalized prior to degeneration in ARL2-Q70L retina. Other studies have demonstrated that shortening of the OS or loss of the connecting cilium results in mislocalization of rhodopsin (Jiang et al., 2015; Hanke-Gogokhia et al., 2016). However, it is important to note that knockout and heterozygote mice for rhodopsin result in the absence of or shorter OS, respectively (Lem et al., 1999; Frederick et al., 2001; Makino et al., 2012). Additionally, mistrafficking of rhodopsin due to transgenic expression of a rhodopsin Pro23His mutant results in misdevelopment of the OS and destabilization of OS discs (Olsson et al., 1992; Haeri and Knox, 2012). Therefore, it is possible that ARL2 disrupts trafficking of rhodopsin thereby affecting OS elaboration. However, the shortening of the IS suggests a different mechanism of defective photoreceptor development because knockout of rhodopsin results in complete loss of the OS, yet they retain inner segments (Lem et al., 1999). Additionally, ARL2- 
Q70L mice have aberrantly localized cilia that do not seem to be targeted to the distal tip of the photoreceptor IS. In either case, it is likely that the slow degeneration of photoreceptors in ARL2-Q70L animals results from accumulation and overload of degradation pathways for rhodopsin (Frederick et al., 2001; Lin et al., 2007; Price et al., 2011).

We demonstrate that expression of ARL2-Q70L leads to stunted growth of ciliary axoneme. ARL2BP, a known interacting protein of ARL2, may potentially be involved in the development of cilia in photoreceptor cells. As previously shown mutation of ARL2BP results in retinitis pigmentosa and one specific mutation, c.134T $>\mathrm{G}$ (p.Met45Arg), disrupts binding of ARL2 (Davidson et al., 2013). Furthermore, knockdown of either ARL2 or ARL2BP results in shortening of cilia in RPE cell lines (Davidson et al., 2013). Notably, the active ARL2-GTP (and not ARL2-GDP) selectively binds to ARL2BP. Therefore, it is expected that ARL2-Q70L would sequester ARL2BP preventing its proper function and resulting in stunted cilia in vivo. Although these data suggest a compelling role for ARL2BP in our model, we do not see an interaction from immunoprecipitation of transgenic retinal lysates. It is possible that our experimental conditions are not sensitive enough or that ARL2BP becomes insoluble after cell lysis and this disrupts the interaction with ARL2.

The photoreceptor cilium is a microtubule based-organelle containing the basal body, connecting cilium, and the axoneme, which extends into the OS. This organelle is the foundation of the photoreceptor OS and therefore necessary for the specialized function of capturing light. Formation of the cilium is complex and is heavily dependent upon the polymerization of tubulin to form microtubules and the primary structure of the OS. Interestingly, ARL2 is a known regulator of microtubules and tubulin in cell culture systems. And more specifically, expression of ARL2-Q70L in multiple cell lines results in destruction of microtubules and cell cycle arrest 
(Bhamidipati et al., 2000; Zhou et al., 2006). In addition, expression of wildtype ARL2 rescues microtubules from tubulin binding cofactor D (TBCD)-mediated destruction (Bhamidipati et al., 2000). A similar result was discovered in the adenocarcinoma cell line, MCF7, where lines with the greatest expression of ARL2 showed higher content of polymerizable tubulin heterodimers and low expressing lines had reduced content (Beghin et al., 2007). A more recent study has also implicated ARL2 in the regulation of soluble polymerizable tubulin pools and showed that this regulation relies on the formation of a "TBC-DEG" chaperone complex composed of ARL2 and the tubulin chaperones TBCD and tubulin binding cofactor E (TBCE), which are proteins necessary for tubulin dimer regulation (Nithiananatham et al., 2015). Considering the major structural defects associated with ARL2-Q70L expression including dysmorphic OS and IS, it is tempting to suggest that ARL2 plays a role in regulation of the polymerizable pool of tubulin in photoreceptors. Moreover, we show an interaction with TBCD a component of the "TBC-DEG" complex mentioned above. Importantly, ARL2BP does not seem to play a role in tubulin regulation, at least outside of its minor effects on ciliary length (Zhou et al., 2006; Newman et al., 2014). We conclude from this that ARL2 may constitutively form "TBC-DEG" and disrupt the normal regulatory function of the complex. How this dysregulation leads to OS and IS dysmophogenesis is unclear and will be the focus of our future study.

Finally, we demonstrate the ARL2-Q70L expression results in aberrant localization of the photoreceptor cilium. Some of the cases of this phenotype can be explained by impending photoreceptor cell death that results in retraction of the OS and IS toward the cell body. However, there were many cases where the basal body and axoneme were localized at the base of the IS protruding from the cell in different directions. Because the transgene is expressed after the point of apical localization of the basal body prior to axoneme elongation, it is likely that 
extension there is a defect in the process of allow this organelle to remain at the apical surface as the IS and OS grows distally. Additionally, we speculate that the defect in axonemal growth is required for proper development of the OS and the cells with mislocalized cilia express the transgene slightly earlier such that they are unable to produce even a rudimentary OS, which results in the growth of the IS over the cilium. Future studies are needed to clarify the role of ARL2 in the process of axoneme elongation.

Major progress has been made in identifying the proteins involved in photoreceptor OS development and ciliogenesis and here we have discovered a novel regulator of these processes. Furthermore, the current data suggest that expression of ARL2-Q70L results in dysregulation of soluble tubulin pools and this dysregulation leads to defects in ciliary development. Our conclusions suggest that regulation of tubulin can affect the architecture of photoreceptor cells including the spatial organization of the cilia, basal body, and ciliary associated centriole. This disruption then results in the misdevelopment of the rod OS and progressive cell death. Future studies are necessary to fully elucidate the mechanisms underlying ARL2 regulation of tubulin and its association with photoreceptor cell development. 


\section{MATERIALS AND METHODS}

\section{RNA isolation and $\mathrm{QRT}$-PCR from retina}

Mouse eyes were enucleated at indicated time points and dissected under microscope (Zeiss Stemi DV4) to isolate the retina. RNA was isolated with TRI reagent (Sigma) according to manufacturer's guidelines. Reverse-transcription PCR reactions containing 0.1-0.5 $\mu$ g RNA were primed with Oligo-dT and random hexamers to generate cDNA. Expression of Arl2 and Arl3 was quantified by SYBR-green qPCR normalized to the expression of Glyceraldehyde-3Phoshpate Dehydrogenase (Gapdh). qRT-PCR primers for genes assessed were: Gapdh Forward $=$ AGACGGCCGCATCTTCTT and Reverse $=$ TTCACACCGACCTTCACCAT, Arl3 - Forward $=$ TACTCCTGGGCTTGGACAAC and Reverse $=$ TGTGACTGCACGCTTTTGAT, Arl2 - Forward $=$ GAGCACCGCGGATTCAA and Reverse $=$ GCAAAGATGAGGAGGGTTCG.

\section{ARL2-Q70L transgenic mouse model generation}

Transgenic model generation followed that of previously published work (Wright et al., 2016). Briefly, a full-length human $A R L 2$ clone (Thermo Scientific) was used generate an $A R L 2$ mutant construct with a tandem C-terminal hemagglutinin (HA) and FLAG tag that is driven by a $4.4 \mathrm{~kb}$ rhodopsin promoter (Rhop). This ARL2 construct was modified by site-directed mutagenesis to produce a glutamine $(\mathrm{Q})$ to leucine $(\mathrm{L})$ variant at position $70(\mathrm{Q} 70 \mathrm{~L})$ using a mutant oligonucleotide (5'-CCG CAG GGA CTT CAG GCC ACC CAC ATC-3'). After amplification, the PCR product was modified with a tandem C-terminal HA and FLAG tag and then cloned behind a $4.4 \mathrm{~kb}$ rhodopsin promoter. All clones selected were sequenced in both directions to confirm that there are no unintended mutations. After removal from the plasmid by digestion, 
Rhop-ARL2-Q70L-polyA was purified by agarose gel electrophoresis and elution. This purified DNA fragment was injected into pronuclei of oocytes from superovulated FVB/N females (WVU Transgenic Core Facilities) and implanted in to pseudo-pregnant CD-1 females.

\section{Genotyping and Founder Line Maintenance}

ARL2-Q70L transgenic founders were initially identified by PCR of genomic DNA isolated from tail snips or ear punches using the following primers: 5'- GGA TCG TGA ATC AGC CTC TGG CTT-3’ and 5’-CTG CAT GCG CTG GCG GTC TGC-3’' PCR reactions utilized NEB quick load Taq polymerase with the following conditions: $1.95^{\circ} \mathrm{C}$ for 2 minutes, $2.95^{\circ} \mathrm{C}$ for 30 seconds, $3.59^{\circ} \mathrm{C}$ for 30 seconds, $4.72^{\circ} \mathrm{C}$ for 45 seconds, and $5.72^{\circ} \mathrm{C}$ for 5 minutes with steps 24 repeated for 33 cycles. Identified founders were crossed with 129/SV-E mice (Charles River) over multiple generations to eliminate the Pde $6 b^{r d 1}$ mutation present in FVB mice (verified by PCR genotyping (Giménez and Montoliu, 2001)) as well as ensure transgenic expression was resulting from a single locus. Animals were maintained under a 12-hour light/12-hour dark cycles with food and water ad libitium. All experimental procedures in this study were approved by the Institutional Animal Care and Use Committee of West Virginia University.

\section{Electroretinographic (ERG) analysis}

Electroretinography (ERG) was performed as previously using UTAS Visual Diagnostic System with BigShot Ganzfeld with UBA-4200 amplifier and interface, and EMWIN 9.0.0 software (LKC Technologies, Gaithersburg, MD, USA) (Wright et al., 2016). After dark-adaptation overnight, a 1:1 mixture of tropicamide:phenylephrine hydrochloride was used to dilate the test animal's eyes. Mice were placed on a heated platform with continuous flow of $1.5 \%$ isofluorane with 2.5 liters per minute oxygen flow rate and a subcutaneous reference electrode. Corneal 
electrodes were placed making contact with the cornea using hypromellose solution ( $2 \%$ hypermellose in PBS) (Gonioscopic Prism Solution, Wilson Ophthalmic, Mustang, OK, USA). Scotopic responses were measured by exposure to flashes of white LED light starting at low intensities and proceeding to higher intensities. Afterward, mice were light-adapted for 10 minutes under $30 \mathrm{~cd} / \mathrm{m}^{2}$ rod-saturating white background light for phototopic response measurement. ERGs

\section{Generation of Antibody against full-length ARL3}

ARL2 antibody was completed as previously (Wright et al., 2016). Briefly, ARL2 with a Cterminal his-tag was expressed in Origami E.coli strain (Novagen). Origami cells were grown to an $\mathrm{OD}_{600} \approx 0.6$ and protein production was induced with $1 \mathrm{mM} \mathrm{IPTG}$ for 18 hours at $18^{\circ} \mathrm{C}$. The protein was purified from the soluble fraction using a Nickel His Affinity Column and the eluate was supplied to Pacific Immunology Corp. for generation of the antibody in rabbits. Antibody serum was then purified against a GST-ARL2 fusion protein and was then tested via western blot and immunofluorescence microscopy in HEK 293 cells expressing ARL2 and retinal extracts.

\section{Immunoblots}

Flash frozen dissected retinal samples from enucleated eyes were sonicated in phosphate buffered saline (PBS) (137 mM NaCl, $2.7 \mathrm{mM} \mathrm{KCl,} 4.3 \mathrm{mM} \mathrm{Na}_{2} \mathrm{HPO}_{4} \cdot 7 \mathrm{H}_{2} \mathrm{O}, 1.4 \mathrm{mM} \mathrm{KH}_{2} \mathrm{PO}_{4}$, with protease inhibitor cocktail (Roche)). After measurement of protein concentration using spectrophotometry (NanoDrop - Thermo Fisher Scientific, Inc), a sample volume equal $150 \mu \mathrm{g}$

total protein was loaded per well of a $12 \%$ or $15 \%$ polyacrylamide SDS-PAGE resolving gel (Criterion Midi format, Bio-Rad). Proteins were then resolved and transferred onto polyvinylidene difluoride membranes (Immunobilon-FL, Millipore, Billerica). Membranes were 
blocked for 30 minutes at room temperature (Western Blot Blocking Buffer - Rockland Inc.) and incubated with primary antibodies at a dilution of 1:2000 overnight at $4^{\circ} \mathrm{C}$. After washing in PBST (PBS with 0.1\% Tween-20) 3 times for 5 minutes, secondary antibodies (goat anti-rabbit Alexa 680 (or 800), goat anti-rat Alexa 800, or goat anti-mouse Alexa 680 (Invitrogen)) were applied for 30 minutes at room temperature. After washing again in PBST, blots were imaged using the Odyssey Infrared Imaging System (LI-COR Biosciences, Lincoln, NE, USA).

\section{Antibodies}

The following antibodies were used in for immunoblot and immunofluorescence analysis at a dilution of 1:2000 or 1:1000, respectively, unless otherwise noted: rabbit anti-PDE6 $\alpha$ (Pierce), rabbit anti-PDE6 $\beta$ (Pierce), rabbit anti-PDE6 $\gamma$ (Pierce), assembled PDE6 (ROS1) (generously provided by Drs. Ted Wensel (Baylor College) and Rick Cote (University of New Hampshire), rabbit anti-Transducin-at1 (Santa Cruz), mouse anti-cytochrome c oxidase subunit I (COX I) (MS404 MitoSciences), rabbit anti-Transducin- $\gamma$ t1 (Santa Cruz), mouse anti-GRK1 (Thermo Fisher), mouse anti-CNGA1 (UC Davis/NIH NeuroMab Facility), rabbit anti-RDS-c (Peripherin) (Gabriel Travis, University of California, Los Angeles, CA), mouse anti-1D4 (rhodopsin) (gift from Dr. Ted Wensel, Baylor Collect of Medicine), rat anti-HA antibody (Roche), chicken antiRP1 (gift from Dr. Qin Liu Mass. Eye and Ear, Boston, MA), and rabbit anti-ARL2 (1:1000) (see above). DAPI (1:1000) (4',6-diamindino-2-phenylindole, Invitrogen) and propidium iodide (PI) (1:2000) (EMD Millipore, Billerica, MA, USA) were also used in indirect immunofluorescence.

\section{Immunoprecipitation (IP)}


Fresh frozen retinal isolates were homogenized in $100 \mu \mathrm{l}$ per retina of PBS containing protease inhibitor (Roche) and $1 \mathrm{mM}$ iodoacetamide. Triton X-100 was added to a final concentration of $1 \%$ and incubated for 1 hour. Debris was removed from the samples by low-speed centrifugation $(10,000 \mathrm{x} g)$ for 10 minutes and the supernatant was incubated with anti-HA affinity matrix (Roche) at $4^{\circ} \mathrm{C}$ for 2 hours. The HA beads were then washed with PBS $+0.1 \%$ TX-100 three times for 5 minutes each. Finally, beads were eluted in SDS sample buffer and immunoblot analysis was performed as above.

\section{Immunofluorescence Analysis}

Indirect immunofluorescence was utilized to analyze protein localization in retinal crosssections. For general immunofluorescence studies, enucleated eyes were immersed in 4\% PFA in PBS for 15 minutes, after which the anterior segments were removed and the eyecups were placed back in fixative for a total of 3 hours. Fixed eyecups were washed with PBS and kept in $20 \%$ sucrose in PBS overnight at $4^{\circ} \mathrm{C}$. After incubation in a 1:1 mixture of $20 \%$ sucrose solution:OCT (Cryo Optimal Cutting Temperature Compound, Sakura) for 2 hours, eyecups were flash frozen in OCT. Alternatively, for staining of ciliary markers anterior segments were dissected immediately after enucleation and eyes were placed in 4\% PFA for 30 seconds. These eyecups were flash frozen in OCT. Serial retinal cross-sections at $16 \mu \mathrm{m}$ thickness (or $10 \mu \mathrm{m}$ for ciliary markers) were mounted on Superfrost Plus slides (Fisher Scientific). Sections were blocked for 1 hour (PBS with 5\% goat serum, 0.5\% Triton X-100, 0.05\% sodium azide) and incubated with primary antibody overnight at $4^{\circ} \mathrm{C}$ then washed with PBS $+0.1 \%$ Triton X-100 three time for 5 minutes. Secondary antibodies (DAPI nuclear stain 405, anti-Rat 568, antiRabbit 488 (or 568), anti-mouse 488 (or 568)) were incubated for 1 hour at room temperature at a dilution of 1:1000. Sections were washed again and cover slipped with ProLong Gold (Life 
Technologies). Confocal imaging was performed using the Nikon C2 Confocal Microscope System. Images were processed using ImageJ image processing software.

\section{Toluidine Blue Immunohistochemical Analysis}

Eyes were enucleated with the 12 o'clock position marked for reference with a red lipophilic dye and immediately fixed in Excalibur Pathology Fixation Buffer at room temperature. Fixed eyes were embedded in paraffin, sectioned at $2 \mu \mathrm{m}$, and stained with toluidine blue by Excalibur Pathology (Norman, OK). Images were captured with a Nikon light microscope. Analysis, including measurement of OS, IS, and ONL length was performed using ImageJ.

\section{Ultrastructural Analysis}

Enucleated eyes fixed in 2\% paraformaldehyde, 2.5\% glutaraldehyde, $0.1 \mathrm{M}$ cacodylate buffer, pH 7.5 for 30 minutes at room temperature were dissected removing the anterior segment and lens then fixed further for at least 2 days. Fixed eyecup was dissected into 6-8 wedge-shaped pieces. Wedges were dehydrated in a graded ethanol series, and then embedded in Polybed 812

(PolySciences, Inc., Warrington, PA, USA). Semi-thin $(1 \mu \mathrm{m})$ sections were collected onto glass slides, stained with toluidine blue, and visualized using a Zeiss Axioimager 2 microscope equipped with EC Plan-Neofluar 40x (N.A. 0.75) and 100x (1.3 N.A.) objectives. Thin sections (ca. $80 \mathrm{~nm}$ ) from selected wedges were collected onto nickel grids, stained with $2 \%$ uranyl acetate and lead citrate, and imaged using an FEI Morgagni transmission electron microscope at $80 \mathrm{kV}$. 


\section{ACKNOWLEDGEMENTS}

We thank Dr. Peter Mathers and Ms. Ingrid Weterrings of the West Virginia University (WVU) Transgenic Animal Core Facility for their help and services in generating our transgenic lines. We thank Drs. Rick Cote, Ted Wensel, and Gabriel Travis for sharing antibodies generated in their laboratories. We are indebted to the members of the Ramamurthy laboratory for their help and support throughout this study.

\section{FUNDING}

This work was supported by grants from the National Institutes of Health (EY017035 (to V.R.), R01EY013246 (to A.F.X.G.), RR017890 (to A.F.X.G.), West Virginia Lions, Lions Club International Fund and an Unrestricted Challenge Grant from Research to Prevent Blindness (RPB). 
CONFLICTS OF INTEREST

None 


\section{REFERENCES}

Beghin A, Honore S, Messana C, Matera EL, Aim J, Burlinchon S, Braguer D, Dumontet C (2007) ADP ribosylation factor like 2 (Arl2) protein influences microtubule dynamics in breast cancer cells. Exp Cell Res 313:473-485.

Bhamidipati A, Lewis S a., Cowan NJ (2000) ADP ribosylation factor-like protein 2 (Arl2) regulates the interaction of tubulin-folding cofactor D with native tubulin. J Cell Biol 149:1087-1096.

Burd CG, Strochlic TI, Gangi Setty SR (2004) Arf-like GTPases: Not so Arf-like after all. Trends Cell Biol 14:687-694.

Davidson AE et al. (2013) Mutations in ARL2BP, encoding ADP-ribosylation-factor-like 2 binding protein, cause autosomal-recessive retinitis pigmentosa. Am J Hum Genet 93:321329 Available at: http://dx.doi.org/10.1016/j.ajhg.2013.06.003.

Frederick JM, Krasnoperova N V, Hoffmann K, Kopish JC, Ru K, Howes K, Lem J, Baehr W (2001) Mutant Rhodopsin Transgene Expression on a Null Background. Invest Ophthalmol 42:826-833.

Giménez E, Montoliu L (2001) A simple polymerase chain reaction assay for genotyping the retinal degeneration mutation (Pdeb(rd1)) in FVB/N-derived transgenic mice. Lab Anim 35:153-156.

Goetz SC, Anderson K V (2010) The primary cilium: a signalling centre during vertebrate development. Nat Rev Genet 11:331-344 Available at: http://dx.doi.org/10.1038/nrg2774.

Haeri M, Knox BE (2012) Rhodopsin mutant P23H destabilizes rod photoreceptor disk 
membranes. PLoS One 7.

Hanke-Gogokhia C, Wu Z, Gerstner CD, Frederick JM, Zhang H, Baehr W (2016) Arf-like protein 3 (ARL3) regulates protein trafficking and ciliogenesis in mouse photoreceptors. J Biol Chem 3:jbc.M115.710954 Available at: http://www.jbc.org/lookup/doi/10.1074/jbc.M115.710954.

Ismail S a, Chen Y-X, Rusinova A, Chandra A, Bierbaum M, Gremer L, Triola G, Waldmann H, Bastiaens PIH, Wittinghofer A (2011) Arl2-GTP and Arl3-GTP regulate a GDI-like transport system for farnesylated cargo. Nat Chem Biol 7:942-949.

Jiang L, Wei Y, Ronquillo CC, Marc RE, Yoder BK, Frederick JM, Baehr W (2015) Heterotrimeric Kinesin-2 (KIF3) Mediates Transition Zone and Axoneme Formation of Mouse Photoreceptors. J Biol Chem 290:12765-12778 Available at: http://www.jbc.org/lookup/doi/10.1074/jbc.M115.638437.

Kahn R a, Volpicelli-Daley L, Bowzard B, Shrivastava-Ranjan P, Li Y, Zhou C, Cunningham L (2005) Arf family GTPases: roles in membrane traffic and microtubule dynamics. Biochem Soc Trans 33:1269-1272.

Lem J, Krasnoperova N V, Calvert PD, Kosaras B, Cameron D a, Nicolò M, Makino CL, Sidman RL (1999) Morphological, physiological, and biochemical changes in rhodopsin knockout mice. Proc Natl Acad Sci U S A 96:736-741.

Li Y, Hu J (2011) Small GTPases and cilia. Protein Cell 2:13-25.

Li Y, Ling K, Hu J (2012) The emerging role of Arf/Arl small GTPases in cilia and ciliopathies. J Cell Biochem 113:2201-2207. 
Lin JH, Li H, Yasumura D, Cohen HR, Zhang C, Panning B, Shokat KM, Lavail MM, Walter P (2007) IRE1 Signaling Affects Cell Fate During the Unfolded Protein Response. 318:944949.

Makino CL, Wen XH, Michaud N a., Covington HI, DiBenedetto E, Hamm HE, Lem J, Caruso G (2012) Rhodopsin expression level affects rod outer segment morphology and photoresponse kinetics. PLoS One 7:1-7.

Mockel A, Perdomo Y, Stutzmann F, Letsch J, Marion V, Dollfus H (2011) Retinal dystrophy in Bardet-Biedl syndrome and related syndromic ciliopathies. Prog Retin Eye Res 30:258-274.

Newman LE, Zhou C, Mudigonda S, Mattheyses AL, Paradies E, Marobbio CMT, Kahn R a. (2014) The ARL2 GTPase Is Required for Mitochondrial Morphology, Motility, and Maintenance of ATP Levels. PLoS One 9:e99270 Available at: http://dx.plos.org/10.1371/journal.pone.0099270.

Nithiananatham S, Le S, Seto E, Jia W, Leary J, Corbett KD, Jeffrey K (2015) Tubulin cofactors and Arl2 are cage-like chaperones that regulate the soluble $\alpha \beta$-tubulin pool for microtubule dynamics. Elife:1-33.

Obata S, Usukura J (1992) Morphogenesis of the photoreeeptor outer segment during postnatal development in the mouse (BALB/c) retina. Cell Tissue Res:39-48.

Olsson JE, Gordon JW, Pawlyk BS, Roof D, Hayes A, Molday RS, Mukai S, Cowley GS, Berson EL, Dryja TP (1992) Transgenic mice with a rhodopsin mutation (Pro23His): A mouse model of autosomal dominant retinitis pigmentosa. Neuron 9:815-830.

Pearring JN, Salinas RY, Baker S a., Arshavsky VY (2013) Protein sorting, targeting and 
trafficking in photoreceptor cells. Prog Retin Eye Res 36:24-51 Available at:

http://dx.doi.org/10.1016/j.preteyeres.2013.03.002.

Price BA, Sandoval IM, Chan F, Simons DL, Wu SM, Wensel TG, Wilson JH (2011)

Mislocalization and degradation of human P23H-rhodopsin-GFP in a knockin mouse model of retinitis pigmentosa. Investig Ophthalmol Vis Sci 52:9728-9736.

Song H, Bush R a, Vijayasarathy C, Fariss RN, Kjellstrom S, Sieving P a (2014) Transgenic expression of constitutively active RAC1 disrupts mouse rod morphogenesis. Invest Ophthalmol Vis Sci 55:2659-2668 Available at: http://www.pubmedcentral.nih.gov/articlerender.fcgi?artid=4001786\&tool=pmcentrez\&ren dertype $=$ abstract.

Taniuchi K, Iwasaki S, Saibara T (2011) BART inhibits pancreatic cancer cell invasion by inhibiting ARL2-mediated RhoA inactivation. Int J Oncol 39:1243-1252.

Tian G, Thomas S, Cowan NJ (2010) Effect of TBCD and its regulatory interactor Arl2 on tubulin and microtubule integrity. Cytoskeleton 67:706-714.

Wätzlich D, Vetter I, Gotthardt K, Miertzschke M, Chen Y-X, Wittinghofer A, Ismail S (2013) The interplay between RPGR, PDE $\delta$ and Arl2/3 regulate the ciliary targeting of farnesylated cargo. EMBO Rep 14:465-472 Available at: http://www.ncbi.nlm.nih.gov/pubmed/23559067.

Wright ZC, Singh RK, Alpino R, Goldberg AFX, Sokolov M, Ramamurthy V (2016) ARL3 regulates trafficking of prenylated phototransduction proteins to the rod outer segment. Hum Mol Genet :1-46 Available at: http://hmg.oxfordjournals.org/content/early/2016/03/28/hmg.ddw077.abstract. 
Zhang Q, Hu J, Ling K (2013) Molecular views of Arf-like small GTPases in cilia and ciliopathies. Exp Cell Res 319:2316-2322 Available at: http://dx.doi.org/10.1016/j.yexcr.2013.03.024.

Zhou C, Cunningham L, Marcus A, Li Y, Kahn RA (2006) Arl2 and Arl3 Regulate Different Microtubule-dependent Processes. Mol Biol Cell 17:2476-2487.

\section{FIGURE LEGENDS}

Figure 1. Endogenous Arl2 Expression Profile and Generation of Dominant Active Mutant Transgenic Model. This figure illustrates the developmental mRNA expression of Arl2 and Arl3 in mouse retina as well as the expression of dominant active ARL2 in our transgenic animal model. . A. Arl2 and Arl3 mRNA expression was determined by quantitative PCR of mouse retinal tissues from P0 to P16. Expression levels are displayed as fold change relative to the expression observed at P0. B. This scheme illustrates the construct used for transgenic mouse generation. Human Arl2 Q70L (ARL2 Q70L; glutamine to leucine dominant active mutant) expression is driven by a $4.4 \mathrm{~Kb}$ rhodopsin promoter (Rhop). The ARL2 Q70L protein is also tagged at the C-terminus with hemagglutinin (HA) and FLAG. C. Western blot analysis of endogenous versus transgenic ARL2 protein levels in P13 retinal tissue samples by staining with ARL2 (red) and HA (green) directed antibodies. Refer to bar graph for quantitation of transgenic expression levels relative to endogenous ( $<<0.01)$. D. Immunofluorescence analysis of retinal cross sections was done at P13 determine the localization of transgenic ARL2 by incubating with HA directed antibody (red) and colocalized with the OS marker peripherin (green) (Scale Bar = 
$20 \mu \mathrm{m}$ ). E. Localization of endogenous ARL2 (using ARL2-directed antibody - green) and the ciliary marker acetylated tubulin (red) (Bottom Panel). (Scale bar $=10 \mu \mathrm{m})$

Figure 2. Decreased photoreceptor function in ARL2-Q70L mice. A. Representative scotopic (rod) and photopic (cone) electroretinograms (ERGs) comparing $\operatorname{Tg}(-)$ and $\operatorname{Tg}(+)$ animals at P16 and P100 across multiple light intensities. B. Graph showing the scotopic a-wave amplitude measured at the light intensity of $-0.8 \log \left(\mathrm{cd}^{*} \mathrm{~s} / \mathrm{m}^{2}\right)$ at different ages. $*=\mathrm{p}<0.01$.

Figure 3. Rod photoreceptor cells degenerate in animals expressing ARL2-Q70L. A. Retinal sections of $\operatorname{Tg}(-)$ and $\operatorname{Tg}(+)$ littermates stained with propidium iodide to demonstrate the ONL integrity at different ages (P13, P60, and P110). B. Quantification of the ONL length (number of nuclei) between $\operatorname{Tg}(-)$ and $\operatorname{Tg}(+)$ littermates at different locations within the retina from the inferior (-3) to superior (3) portion at P13 and P110. ${ }^{*}=\mathrm{p}<0.01$ (Scale Bar $=20 \mu \mathrm{m}$ ).

Figure 4. Reduction of photoreceptor OS proteins in ARL2-Q70L animals. Representative immunoblots showing expression of proteins from retinal lysates of $\operatorname{Tg}(-)$ and $\operatorname{Tg}(+)$ littermates at P13. Quantification of these protein levels is shown in the bar graph on the right. All samples were normalized to $\mathrm{G} \alpha_{0}$, a protein expressed in the bipolar cells. * $=\mathrm{p}<0.05$.

Figure 5. Abnormal elaboration of photoreceptor OS in ARL2-Q70L Mice. Representative toluidine blue stained retinal cross sections from $\operatorname{Tg}(-)$ and $\operatorname{Tg}(+)$ littermates at P13 and P16. Quantification of the OS, IS and ONL length from these animals at respective ages. ${ }^{*}=\mathrm{p}<0.05$. (scale bar $=20 \mu \mathrm{m})$.

Figure 6. Mislocalization of Rhodopsin in ARL2-Q70L animals. Immunofluorescence analysis of OS proteins in $\operatorname{Tg}(-)$ and $\operatorname{Tg}(+)$ samples at P13. PDE6 $\beta$ (A), Rhodopsin (B), Gat1 
(C), and GRK1 (D) with unadjusted image intensity (Top) and enhanced image intensity to visualize immunoreactivity in the ONL (Bottom). (Scale Bar $=20 \mu \mathrm{m})$.

Figure 7. ARL2-Q70L expression results in defective ciliogenesis and OS development in rod photoreceptor cells. A. Immunofluorescence analysis of retinal cross sections of Tg(-) and $\operatorname{Tg}(+)$ littermates showing immunoreactivity for DAPI (blue), RPGR (green) and RP1 (magenta) (Scale Bar $=10 \mu \mathrm{m})$. A frequency distribution illustrating the fraction of total measured cilia (length from base of RPGR to tip of RP1 immunoreactivity) ranging between 0-1, 1-2, 2-3, 3-4, 4-5, and 5+ microns (White arrows indicate mislocalized cilia). B. Electron micrographs of retinal cross sections at low magnification of $\operatorname{Tg}(-)$ and $\operatorname{Tg}(+)$ littermates at P13 illustrating the structural abnormalities of the IS, OS, and cilium in $\operatorname{Tg}(+)$ (Scale Bar $=2 \mu \mathrm{m})$. C. High magnification electron micrographs highlighting ultrastructural defects. Black arrows indicate misoriented or mislocalized basal bodies and cilia. White arrowheads indicate misoriented/dysmorphic OS discs (Scale Bar $=0.5 \mu \mathrm{m})$.

\section{Figure 8. Tubulin binding cofactor D (TBCD) interacts with ARL2 in vivo.}

Immunoprecipitation (IP) of retinal isolates from $\mathrm{Tg}(-)$ and $\mathrm{Tg}(+)$ ARL2-Q70L using an HAdirected affinity resin. Immunoblots from IP samples were probed with indicated antibodies. $\mathrm{T}=$ Total, $\mathrm{U}=$ Unbound, and $\mathrm{E}=$ Eluate. 


\section{ABBREVIATIONS}

ARL2 = ADP-Ribosylation Factor-Like 2

ARL2-Q70L = transgenic dominant active ARL2

TBCD = tubulin binding cofactor D

PDE6 $=$ Phosphodiesterase 6

PDE6 $\alpha=$ phosphodiesterase, alpha subunit

PDE6 $\beta=$ phosphodiesterase, beta subunit

PDE $\gamma=$ phosphodiesterase, gamma subunit

GRK1 = Rhodopsin Kinase (G-protein receptor kinase 1)

$\mathrm{RP}=$ Retinitis pigmentosa

$\mathrm{XLRP}=\mathrm{X}$-linked recessive retinitis pigmentosa

DAPI = 4',6-diamidino-2-phenylindole

ERG $=$ electroretinogram

OS = outer segment

ROS $=$ rod outer segment

COS $=$ cone outer segment

IS $=$ inner segment

RIS $=$ rod inner segment

CIS $=$ cone inner segment

ONL = outer nuclear layer

INL = inner nuclear layer

CNGA1 = cyclic nucleotide gated channel 
Figure 1
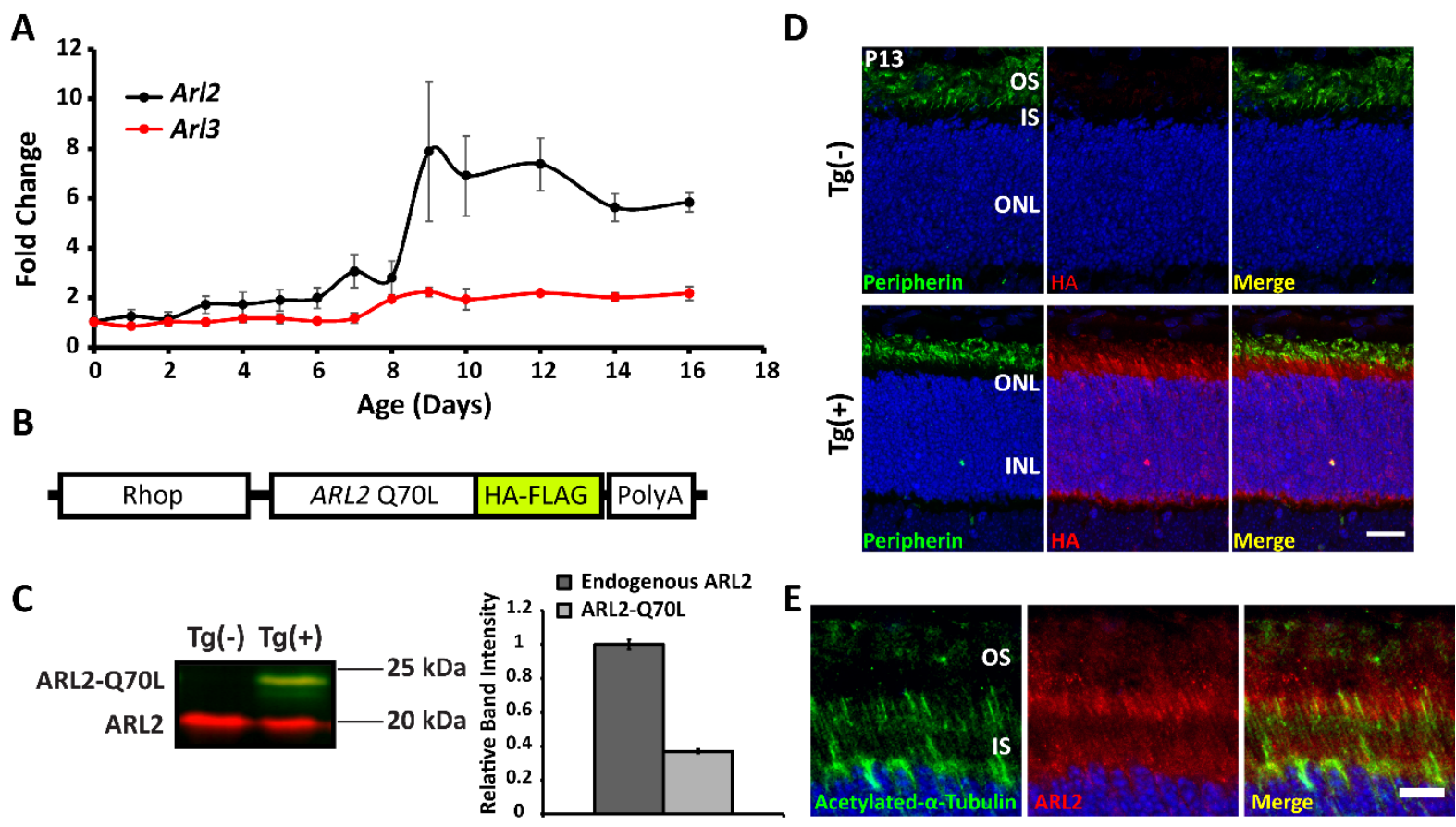
Figure 2
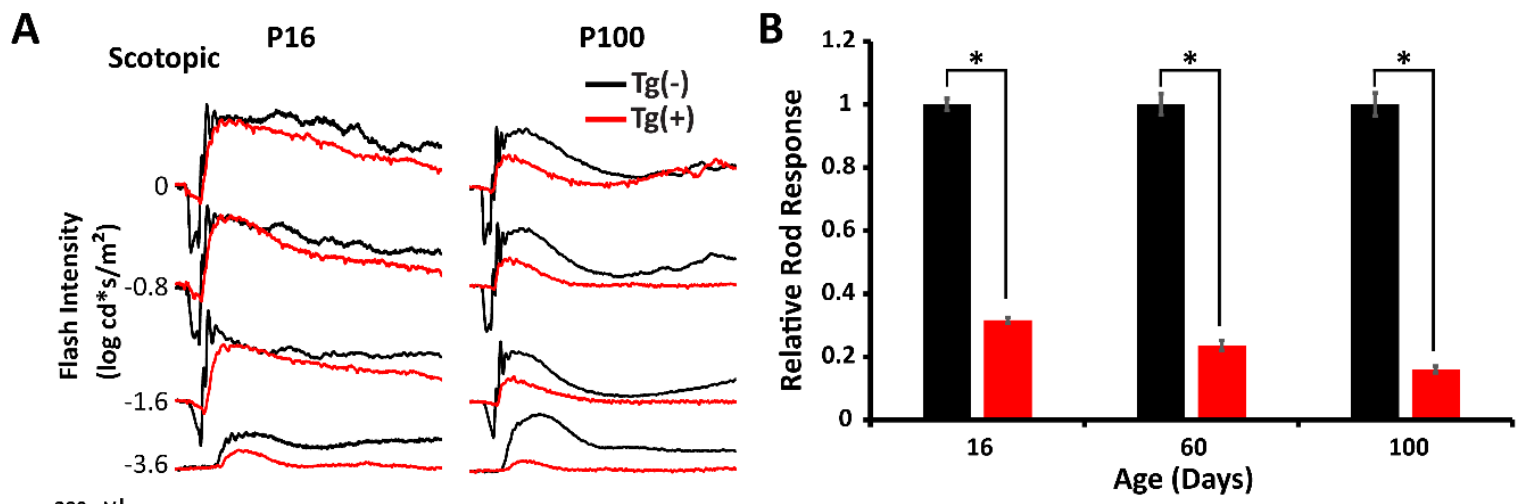

$200 \mu \mathrm{VL}$

Photopic

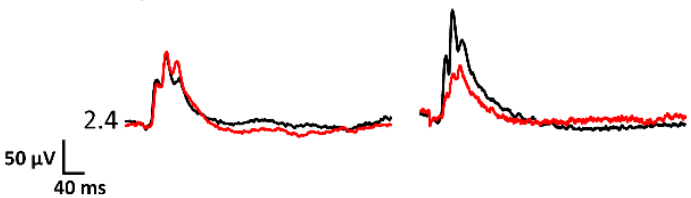


Figure 3

A
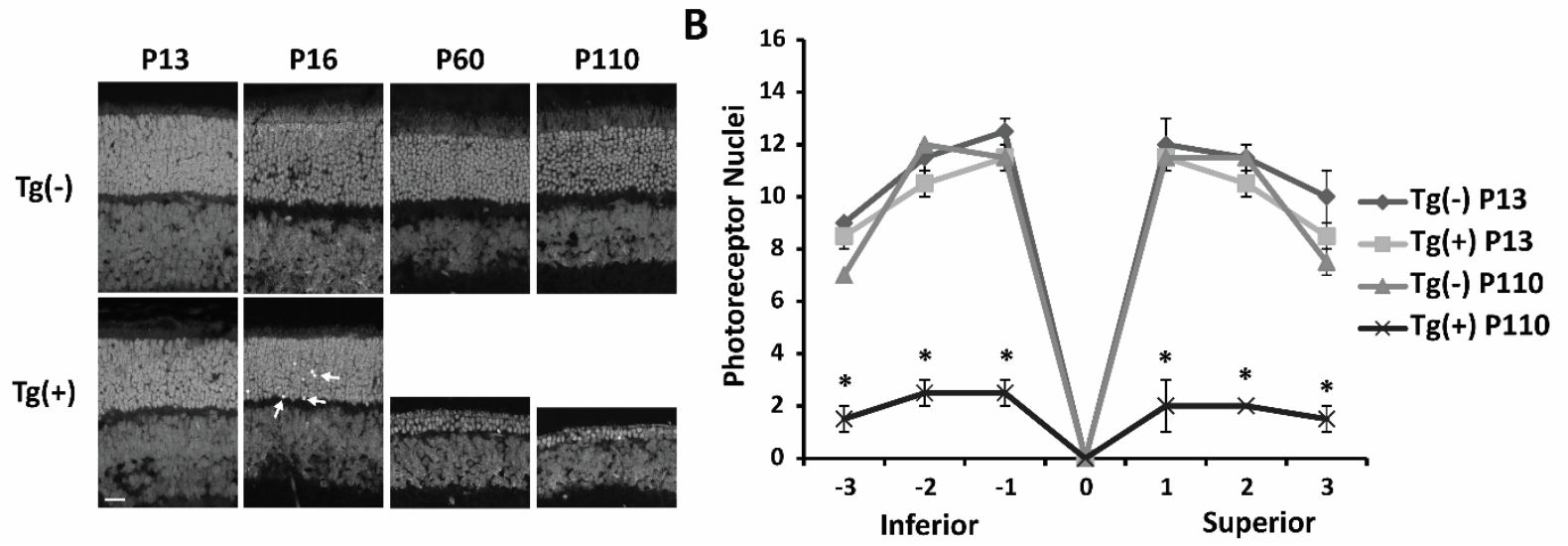
Figure 4
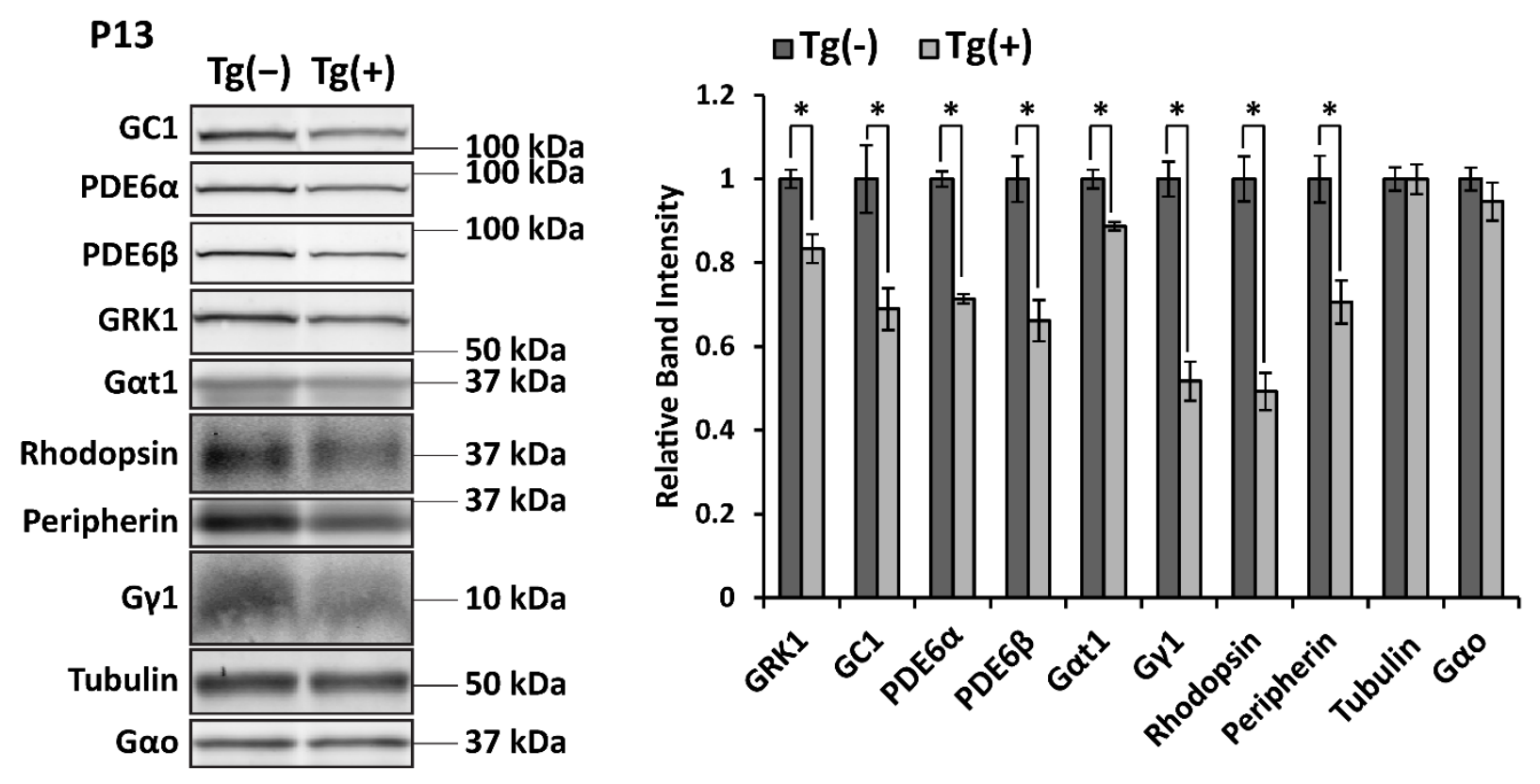
Figure 5
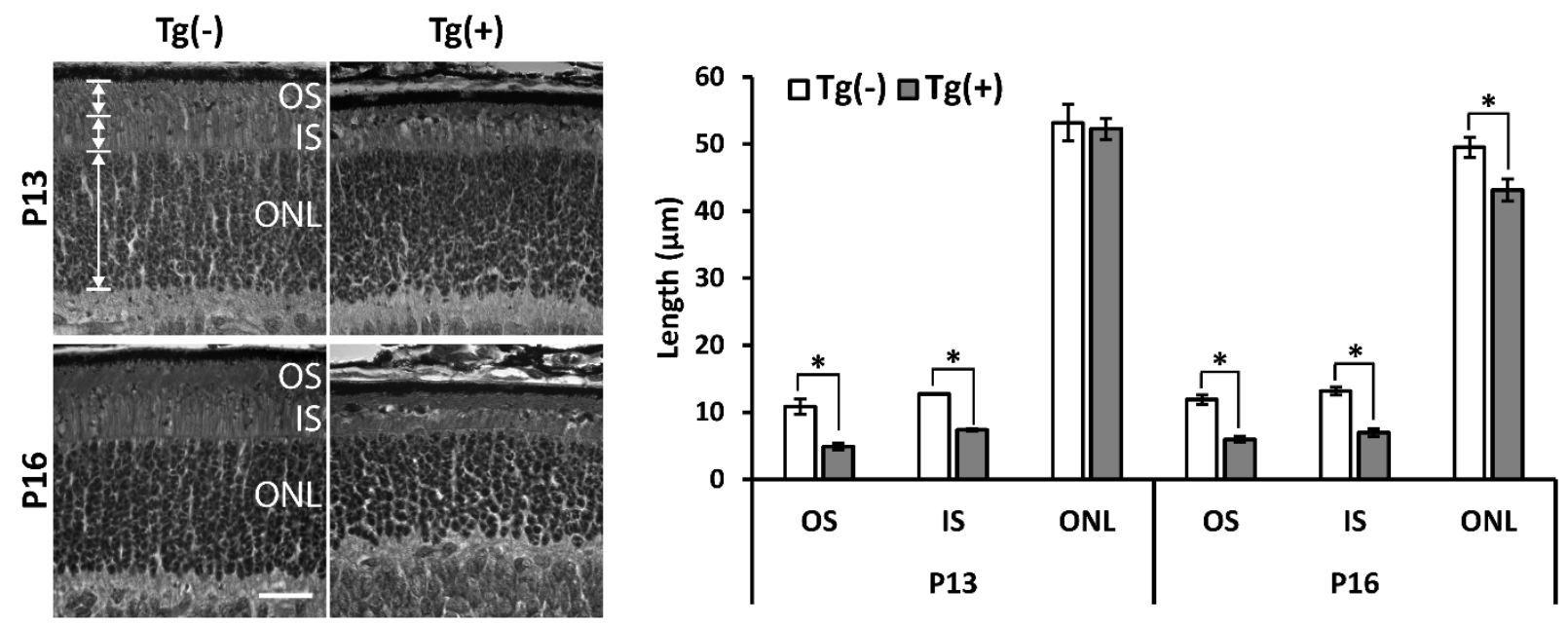
Figure 6

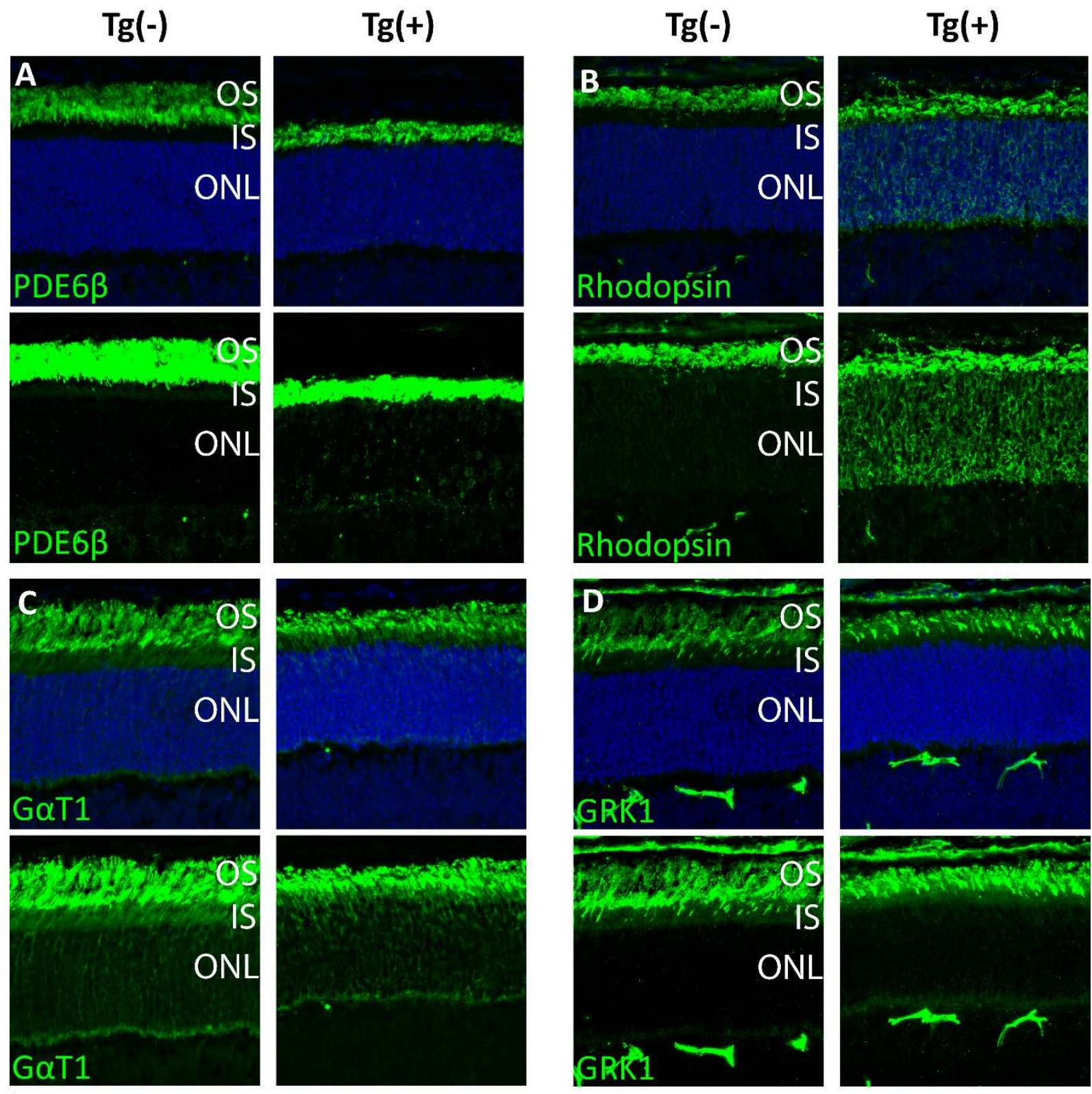

$20 \mu \mathrm{m}$ 
Fig. 7

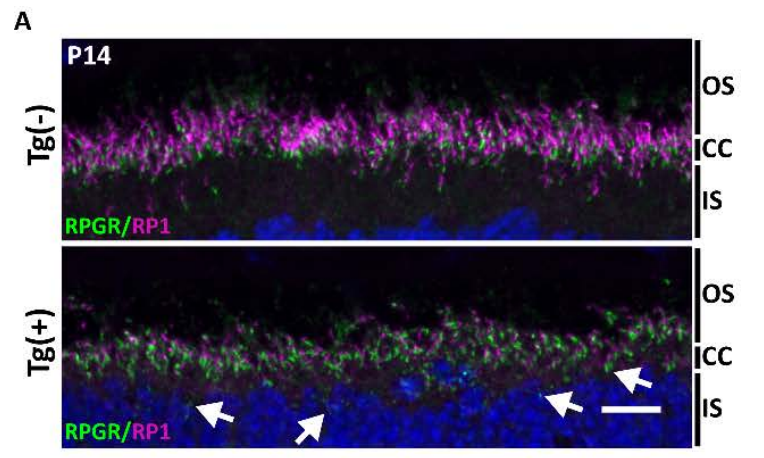

B

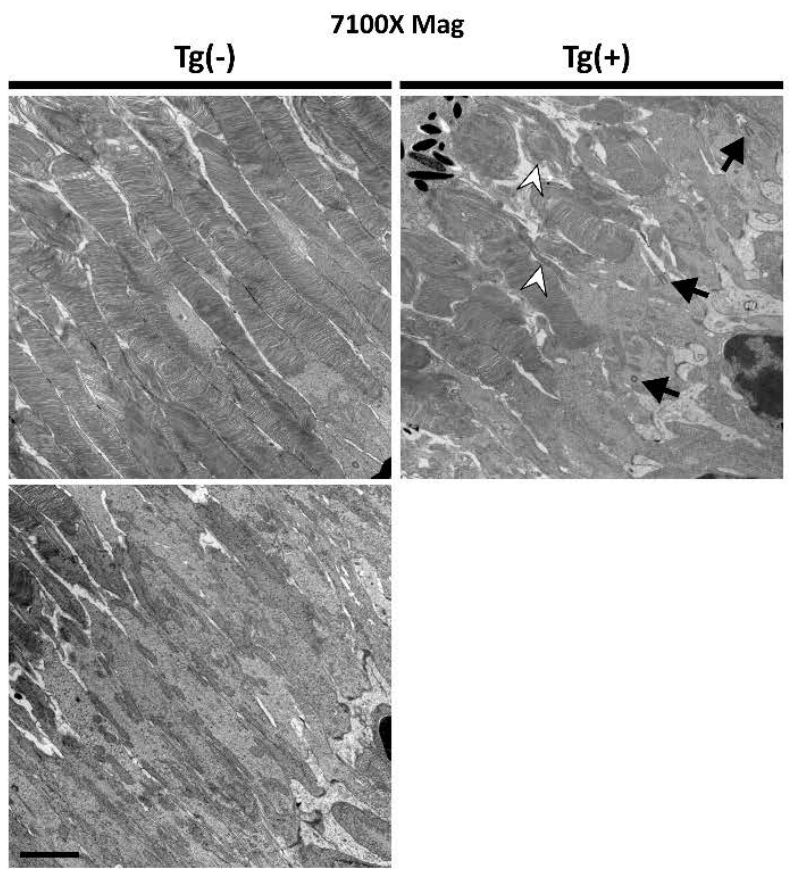

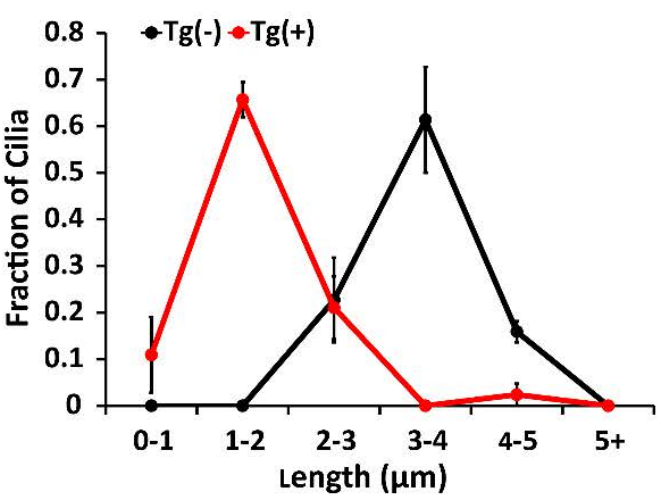

C

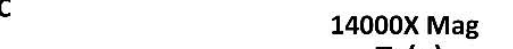
$\mathrm{Tg}(+)$

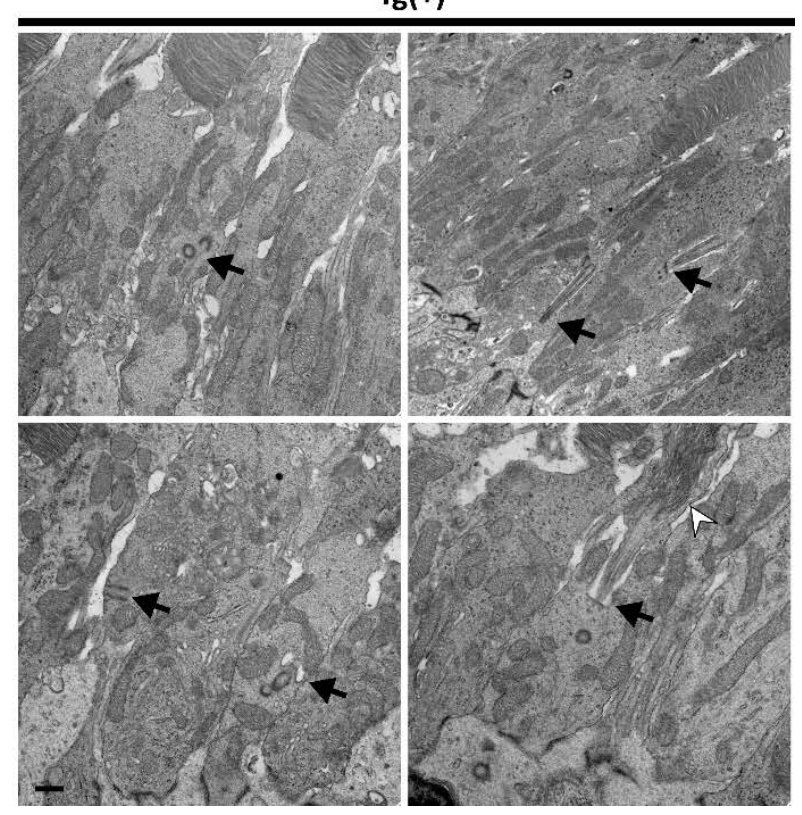


Figure 8

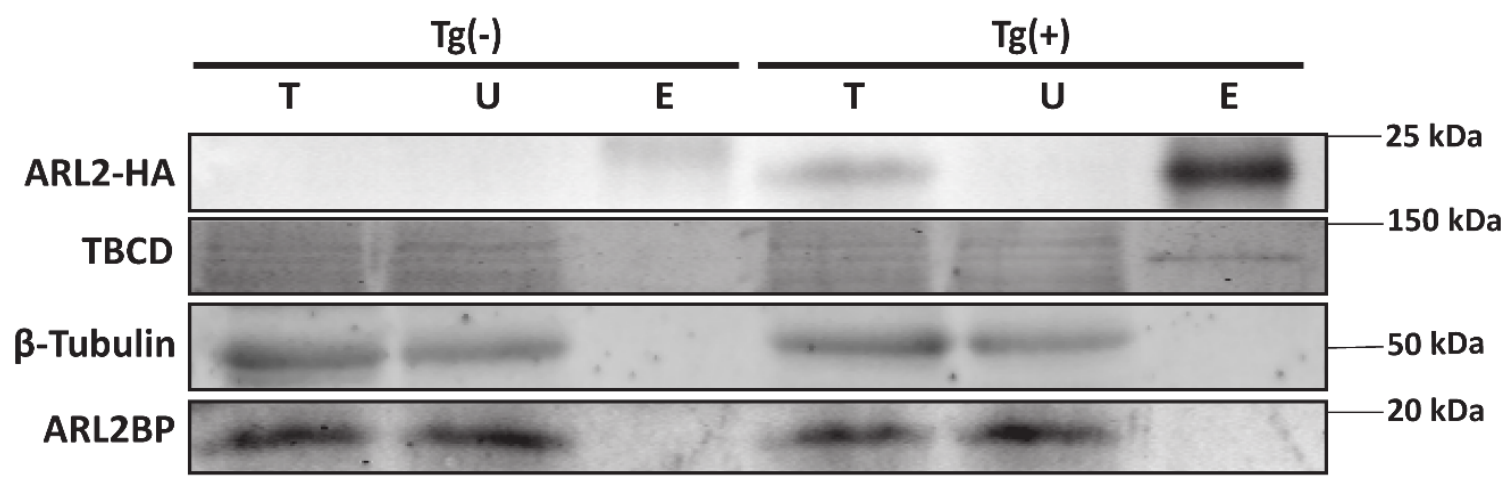




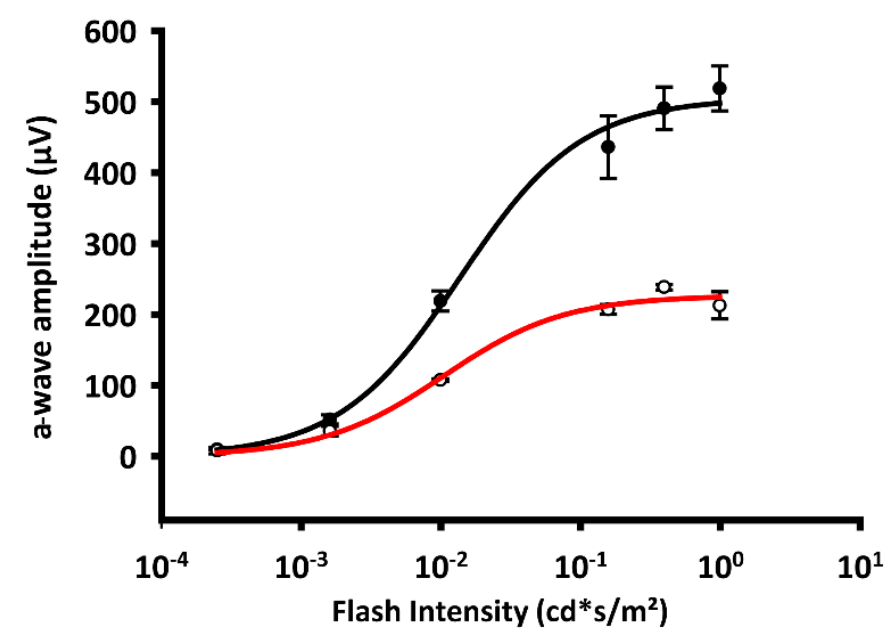

Supplementary Figure 1: Intensity-response relationships for scotopic a-waves recorded at

P16. The data were fitted with a hyperbolic function to determine a-wave half-saturating light intensities of $0.010 \pm 0.0019 \mathrm{~cd}^{*} \mathrm{~s} / \mathrm{m}^{2}$ in $\mathrm{Tg}(+)$ animals versus $0.014 \pm 0.0018 \mathrm{~cd} * \mathrm{~s} / \mathrm{m}^{2}$ in littermate controls and calculated maximum a-wave amplitudes of $225.2 \pm 6.6 \mu \mathrm{V}(\mathrm{Tg}(+))$ and $499.7 \pm 10.6 \mu \mathrm{V}(\operatorname{Tg}(-))$ 

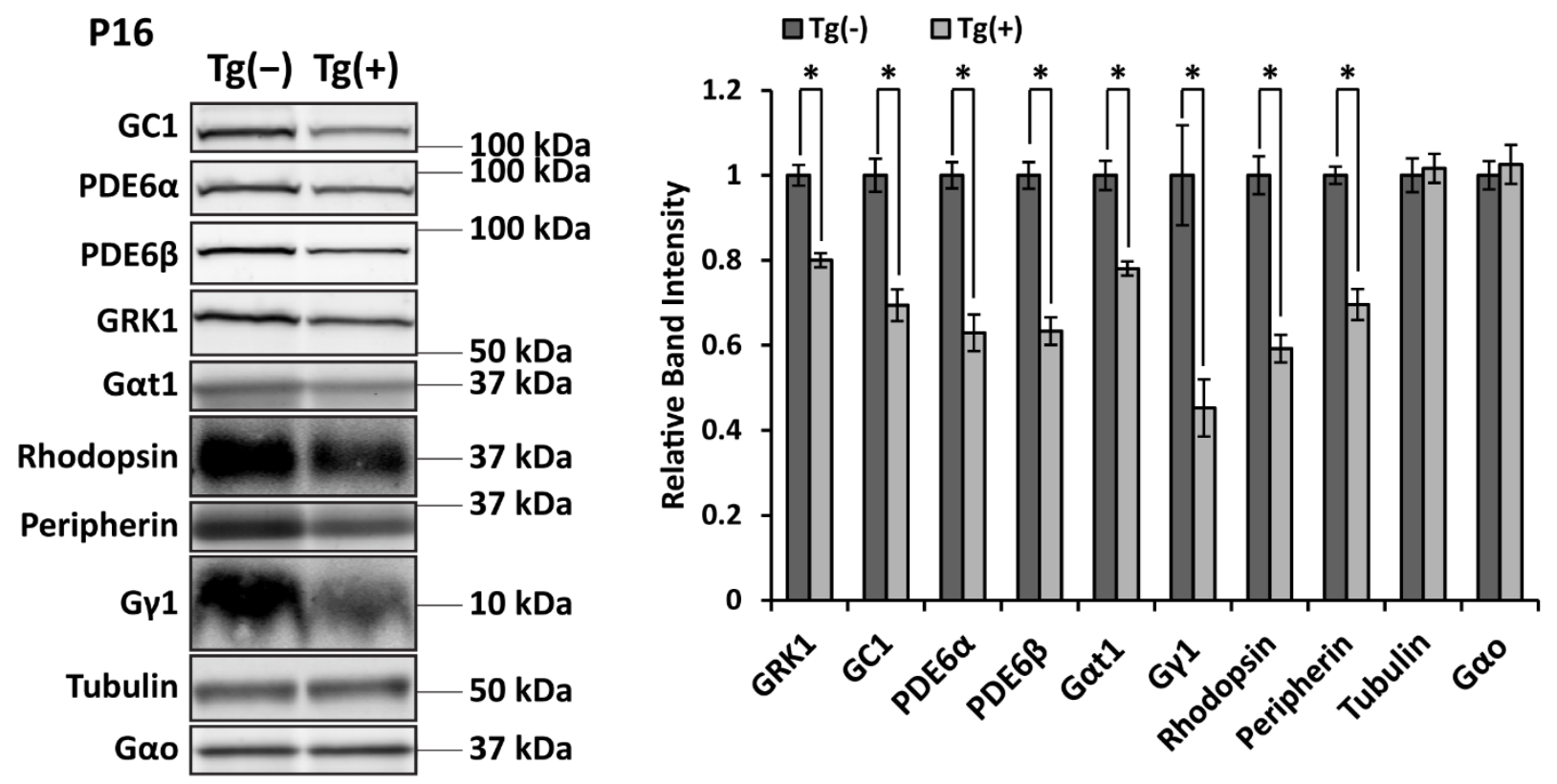

Supplementary Figure 2. Pan-reduction in photoreceptor OS proteins ARL2-Q70L

animals. Representative immunoblots showing expression of proteins from retinal lysates of $\operatorname{Tg}(-)$ and $\operatorname{Tg}(+)$ littermates at P16. Quantification of these protein levels is shown in the bar graph on the right. All samples were normalized to $\mathrm{G} \alpha_{0}{ }^{*}=\mathrm{p}<0.05$. 

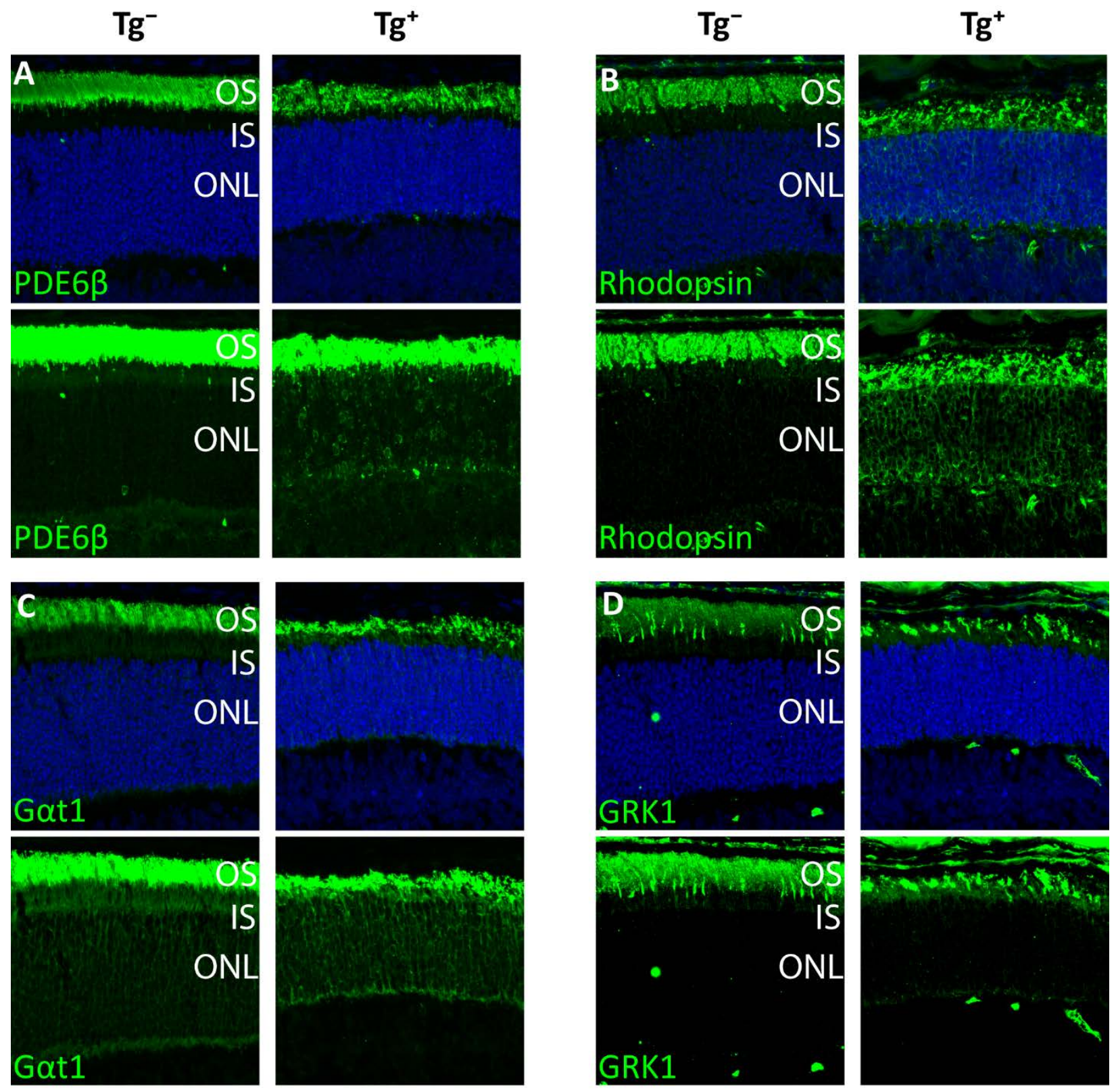

Supplementary Figure 3. Mislocalizatoin of Rhodopsin in ARL2-Q70L animals.

Immunofluorescence analysis of OS proteins in $\operatorname{Tg}(-)$ and $\operatorname{Tg}(+)$ samples at P16. PDE6 $\beta$ (A.),

Rhodopsin (B.), Gat1 (C.), and GRK1 (D.) with unadjusted image intensity (Top) and enhanced image intensity to visualize immunoreactivity in the ONL (Bottom). (Scale Bar $=20 \mu \mathrm{m})$. 


\section{Chapter 4: Summary and Future Directions}

Photoreceptor cell development and function requires highly complex spatial and temporal regulation of protein:protein interactions. There are relatively few insights into how these interactions are regulated, however, we do know that they often require the help of small GTPases. Although there have been a number of discoveries, research into the role of these proteins in photoreceptor cells is in its infancy. Moreover, there are a growing number of discoveries linking small GTPases with photoreceptor mediated disease, such as ARL6, ARL2, ARL3, and RAB28 (refer to Chapter 1). The purpose of my dissertation was to characterize the regulation of photoreceptor cell protein trafficking and OS development by the two small GTPases, ARL2 and ARL3.

ARL2 and ARL3 are highly homologous proteins sharing 53\% identity in primary sequence and a high degree of structural similarity in addition to sharing multiple binding partners. Using site-directed mutagenesis and transgenesis, we identified the processes that these proteins are associated with in vivo. I created multiple animal models including an ARL3-Q71L dominant active transgenic model and an ARL3-WT transgenic model (Chapter 2) as well as an ARL2-Q70L dominant active transgenic model (Chapter 3). Through these models we determined that ARL2 and ARL3 serve different primary functions in photoreceptor cells despite having similar binding partners and sequences. Specifically, ARL3 appears to be primarily a regulator of trafficking of prenylated proteins to the outer segment, while ARL2 is a crucial component of photoreceptor cilia development.

The combination of my research (Chapter 2) and the work of Baehr and colleagues, we now have a better understanding of the function of ARL3 in vivo. However, these models do 
have some discrepancies based on the timing of gene disruption or transgenic expression. Specifically, embryonic knockout of Arl3 results in improper OS development and early cell death while late knockout or expression of a dominant active ARL3 (ARL3-Q71L) (after P4) results in defects in prenylated protein trafficking and progressive cell death. My studies have implicated sequestration of prenyl binding proteins, like $\operatorname{PrBP} \delta$, as the underlying cause of prenylated protein trafficking defects, yet we have no clear mechanism underlying the more sever defects from early loss of Arl3. Additionally, ARL3-Q71L expression, but no knockout of Arl3, results in defective migration of photoreceptor neurons to their proper location, i.e. the outer nuclear layer (ONL). The unexplained misdevelopment and early degeneration as well as the nuclear migration defect observed in these models provide the primary questions to pursue for future research of ARL3.

Understanding the discrepancy between early and late degeneration in the knockout models will require a better understanding of the role of ARL3 in OS formation or the primary underlying process, ciliogenesis. More specifically, the other proteins associated with ARL3 in this process must be identified. Although we have performed proteomic analysis from using dominant active ARL3 immunoprecipitation from retinal samples, we have yet to identify novel binding partners that could explain this phenotype. It is possible that the primary target of ARL3 the time of OS development is not expressed in adulthood when we performed the immunoprecipitation experiments. More extensive proteomic analysis during this timeframe may yield novel binding partners that help explain defects in development. In addition, it was recently shown that ARL13b can act as a guanine nucleotide exchange factor (GEF) for ARL3, and may be responsible for activation of ARL3 in vivo. This would explain the discrepancy in phenotype between Arl3 gene disruption and expression of a dominant active protein. Specifically, the 
interaction of ARL13b and ARL3 may be crucial for OS development. This can be studied by genetic models that result in disrupted interaction of these proteins or simply loss of ARL13b.

The pathway through which ARL3 acts to affect nuclear migration has not been identified and the phenotype as not been fully characterized in vivo. Further studies must focus on earlier expression of dominant active ARL3 or the combination of endogenous Arl3 knockout and expression of ARL3-Q71L. These approaches will produce the most severe phenotype, which will provide more insight into the processes involved. It is possible that all cells require ARL3 function to have proper nuclear migration. Temporal snapshots can be collected and the timing and pattern of migration can be observed. Further proteomic analysis during this timeframe may also be required to understand the components involved. Importantly, ARL3 is ubiquitously expressed and therefore may play a role in nuclear migration in the cortex. This provides an area to expand the research of ARL3 function.

While the function of ARL3 in vivo has been elucidated in more detail by my own studies and previous research from other laboratories, the mechanisms underlying ARL2 regulation of photoreceptor development remain very much unclear. Our data suggest that ARL2 may play a role in regulation of ciliary growth and localization in photoreceptor cells and subsequently affect the morphogenesis of the OS. However, we have yet to determine the details underlying these phenotypes. Proteomic analysis of ARL2 binding partners from the dominant active animal model may provide insight into the molecular pathway that it is involved in during ciliogenesis. Additionally, future experiments must utilize in vitro techniques to determine the function of ARL2 on ciliary growth and dynamics. These experiments can be used to explain the potential role for ARL2 regulation of TBCD and ARL2BP in the process of ciliogenesis. ARL2 may also play a role in energy metabolism by acting at mitochondria in photoreceptor cells. However, it is 
unclear whether disruption of ATP levels would affect ciliogenesis in photoreceptors. Because axonemal growth and microtubule maintenance is an energy dependent process it is certainly possible. Further investigation into the function of ARL2 at mitochondria must be completed. Finally, as we have seen in the case of ARL3, embryonic knockout may result in a different phenotype than a transgenic expression of a dominant mutant, therefore it will be fruitful to examine a conditional mouse knockout model of ARL2.

While this research has established a foundation for the role of ARL2 and ARL3 in photoreceptor cells, there are many questions left to be answered. The elucidation of the molecular cascades in which these proteins are involved will result in a better understanding of ciliogenesis and protein trafficking. This understanding will provide the information necessary for the creation of unique therapeutics targeting the underlying mechanisms of photoreceptor mediated blinding disease. 